\title{
Pesticide Options for Important Insect, Mite and Mollusk Pests of Commercial Flowers in Florida ${ }^{1}$
}

James F. Price, Curtis Nagle, and Elzie McCord, Jr. ${ }^{2}$

Effective arthropod pest management in commercial floral crops requires that pests be detected in a timely manner through systematic scouting and that appropriate control measures be applied as conditions warrant. Control measures may include economical combinations of cultural controls (such as disposal of crop residue, reducing unnecessary water in the production environment, selection of less affected varieties and species, etc.), physical controls (such as screen barriers, hot water treatments of certain planting stock, hand removal of pests, etc.), biological controls (such as release of beneficial parasites, predators, and pathogens to attack the pests) and chemical controls. This insect control guide is a summary of chemical control measures that are presently available to commercial flower producers in Florida and includes beneficial nematode and microbial insecticides that also are components of biological control.

The tables in this document list pesticides whose labels provide for the product's use on any "ornamental" or "flower" crop. Products whose label permits use on named flower or ornamental crops only are not summarized here but may appear in other EDIS publications relating to pest management in those specific crops. Tables also indicate the permitted production site, either greenhouse $(\mathrm{G})$ or field or shade house $(\mathrm{F})$ as well as notes taken from the labels to qualify some uses. Usually only one example of each formulation is given; however, there may be other products as effective as those listed. The tables are organized alphabetically by active ingredient and address the following major pest groups:

Aphids

Armyworms (see also Caterpillars)

Caterpillars (see also: Armyworms,

Heliothis \& Helicoverpa,

Leafrollers \& Leaftiers, Loopers)

Chrysomelid Beetles

Fleahoppers

Fungus Gnats

1. This document is ENY-695, one of a series of the Entomology \& Nematology Department, Florida Cooperative Extension Service, Institute of Food and Agricultural Sciences, University of Florida. Published: December 2003. Revised: September 2004. For more publications related to horticulture/agriculture, please visit the EDIS Website at http://edis.ifas.ufl.edu/.

2. James F. Price, associate professor, Curtis Nagle, Elzie McCord, Jr., Entomology and Nematology Department, Gulf Coast Research and Education Center, Bradenton, 34203.

The Institute of Food and Agricultural Sciences (IFAS) is an Equal Employment Opportunity - Affirmative Action Employer authorized to provide research, educational information and other services only to individuals and institutions that function without regard to race, creed, color, religion, age, disability, sex, sexual orientation, marital status, national origin, political opinions or affiliations. For information on obtaining other extension publications, contact your county Cooperative Extension Service office. Florida Cooperative Extension Service / Institute of Food and Agricultural Sciences / University of Florida / Larry R. Arrington, Interim Dean 
Heliothis \& Helicoverpa (see also Caterpillars)

Leafhoppers

Leafminers

Leafrollers \& Leaftiers (see also Caterpillars)

Loopers (see also Caterpillars)

Mealybugs

Mites, Broad \& Cyclamen

Mites, Eriophyid

Mites, False Spider (privet mites)

Mites, Spider

Plant (Lygus) Bugs

Shore Flies

Slugs \& Snails

Thrips

Whiteflies

Precautionary statements, re-entry intervals (REI), restricted use status, and registrants are provided in a separate publication, Insecticides, Miticides \& Molluscicides Available to Florida's Floricultural Industry (ENY-679)

(http://edis.ifas.ufl.edu/IN472), to aid in the selection and safe use of the pesticides. When available, label hyperlinks are provided in the electronic forms of that document.

The product label communicates the lawful use of the product and must be read, understood and followed. The label contains important limitations that may not be presented in these tables. When using a pesticide for the first time, it is important to apply the product first to a small portion of the crop and wait to observe possible detrimental effects, such as leaf distortion and plant stunting.

Many pesticides decompose in the spray tank when mixed with water above $\mathrm{pH}$ 7. Growers should test the $\mathrm{pH}$ of their water and when above 7 , should add a buffering solution to maintain $\mathrm{pH}$ between 6.5 and 7.
This summary is only a guide to aid in selection of pesticides. Care has been given to provide accurate and up-to-date information, but it is possible that, through label changes, etc., improper uses may be indicated. In all cases it is the responsibility of the applicator to read, understand, and comply with the label that accompanies each pesticide container.

As an additional precaution, keep the telephone number and address of the nearest county poison control center in a convenient location in case of an accidental poisoning. Also, keep clean copies of labels of all pesticides on your premises. In the event of a poisoning, the label of the pesticide involved should accompany the victim to the poison control center or hospital. 
Table 1. Aphid (Homoptera: Aphididae) control measures available to commercial producers of any flower crop in Florida.

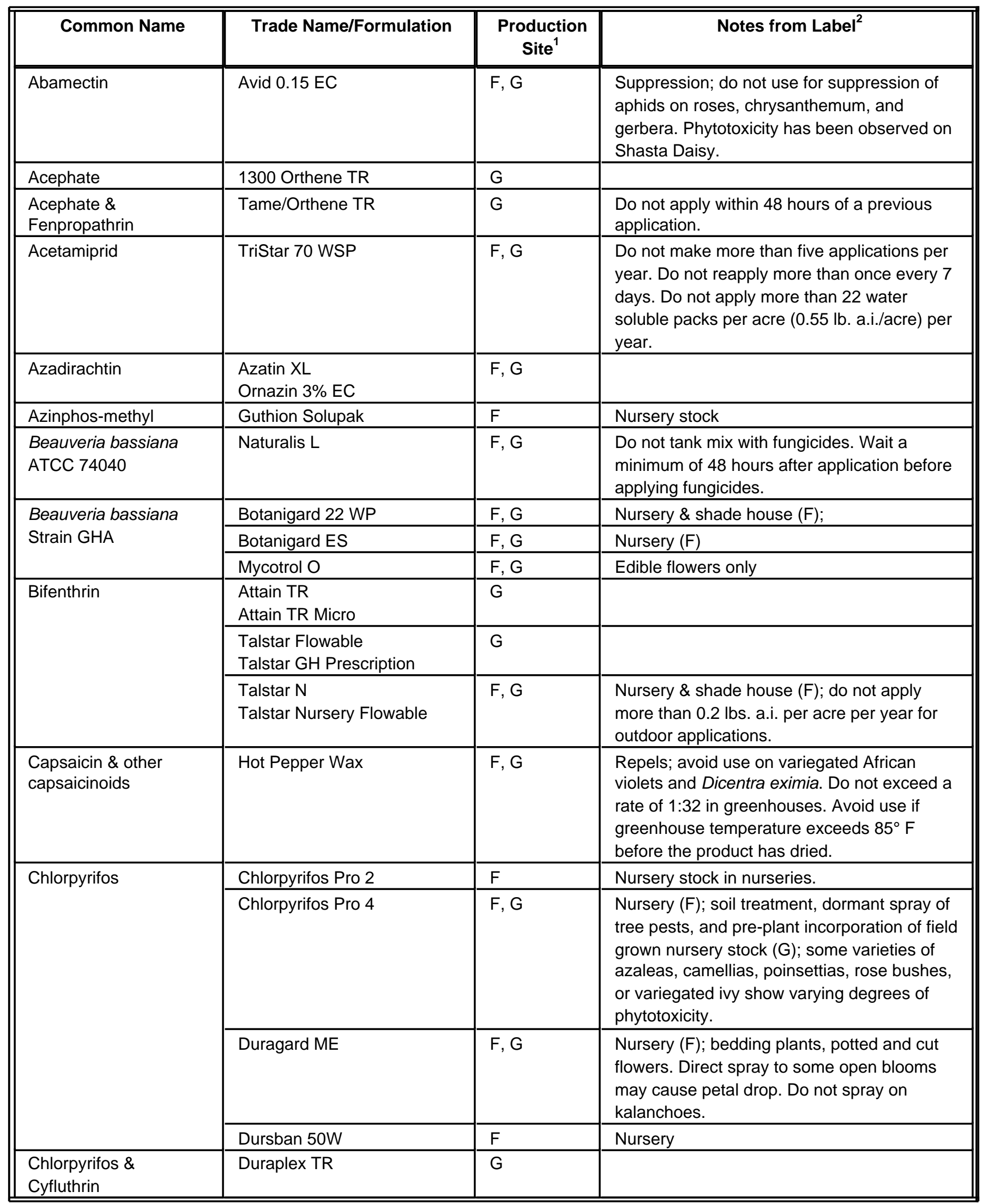


Table 1. Aphid (Homoptera: Aphididae) control measures available to commercial producers of any flower crop in Florida.

\begin{tabular}{|c|c|c|c|}
\hline Common Name & Trade Name/Formulation & $\begin{array}{l}\text { Production } \\
\text { Site }^{1}\end{array}$ & Notes from Label ${ }^{2}$ \\
\hline $\begin{array}{l}\text { Clarified Hydrophobic } \\
\text { Extract of Neem Oil }\end{array}$ & Triact 70 & $F, G$ & $\begin{array}{l}\text { Test before applying to carnation and the } \\
\text { flowers of: Impatiens, fuchsia, hibiscus, and } \\
\text { some roses. Most effective when applied } \\
\text { early to mid-morning or late afternoon. }\end{array}$ \\
\hline Cyfluthrin & Decathlon $20 \mathrm{WP}$ & $F, G$ & \\
\hline Deltamethrin & Deltagard GC 5 SC & $\mathrm{F}$ & Nursery \\
\hline \multirow[t]{2}{*}{ Diazinon } & $\begin{array}{l}\text { Diazinon } 50 \mathrm{~W} \\
\text { Diazinon } \mathrm{Ag} 500\end{array}$ & $\mathrm{~F}$ & $\begin{array}{l}\text { Precaution: Do not use on ferns, poinsettias, } \\
\text { hibiscus, pilea, and gardenia since injury to } \\
\text { the plants may occur. }\end{array}$ \\
\hline & KnoxOut NL & $\mathrm{F}$ & Nursery \\
\hline Endosulfan & $\begin{array}{l}\text { Endosulfan } 3 \text { EC } \\
\text { Endosulfan } 50 \text { WP } \\
\text { Thionex (Endosulfan) } 3 \text { EC } \\
\text { Thionex (Endosulfan) } 50\end{array}$ & $F, G$ & $\begin{array}{l}\text { Do not exceed } 3.0 \text { lbs. a.i. per acre per year. } \\
\text { On chrysanthemums, best results will be } \\
\text { obtained if applied before plants flower. }\end{array}$ \\
\hline Fenoxycarb & Afeclude TR & $G$ & \\
\hline Fenpropathrin & Tame 2.4EC & $F, G$ & $\begin{array}{l}\text { Nursery \& shade house }(\mathrm{F}) \text {; do not use less } \\
\text { than a } 7 \text { day re-treatment interval. }\end{array}$ \\
\hline \multirow[t]{3}{*}{ Imidacloprid } & Marathon $1 \%$ Granular & $F, G$ & $\begin{array}{l}\text { Nursery }(\mathrm{F}) \text {; for outdoor ornamentals grown } \\
\text { in beds, applications can not exceed a total of } \\
40 \mathrm{lbs} \text {. per acre per year. On plants with a } \\
\text { production cycle of less than } 1 \text { year, } \\
\text { application is not to exceed a frequency of } \\
\text { more than once each } 16 \text { weeks for a } \\
\text { particular plant. On stock plants and woody } \\
\text { crops with a production cycle of greater than } \\
1 \text { year, application may not exceed once a } \\
\text { year. }\end{array}$ \\
\hline & Marathon 60 WP & $F, G$ & $\begin{array}{l}\text { Nursery }(\mathrm{F}) \text {; this product is to be applied as a } \\
\text { soil treatment only. For outdoor ornamentals } \\
\text { grown in beds applications can not exceed a } \\
\text { total of } 10.7 \text { oz. ( } 0.4 \mathrm{lbs} \text {. active ingredient) per } \\
\text { acre per year. On plants with a production } \\
\text { cycle of less than } 1 \text { year, application is not to } \\
\text { exceed a frequency of more than once each } \\
16 \text { weeks for a particular plant. On stock } \\
\text { plants and woody crops with a production } \\
\text { cycle of greater than } 1 \text { year, application may } \\
\text { not exceed once a year. }\end{array}$ \\
\hline & Marathon II & $F, G$ & $\begin{array}{l}\text { For outdoor ornamentals do not apply more } \\
\text { than } 1.6 \text { pt. ( } 0.4 \mathrm{lbs} \text {. a.i.) per acre per year. } \\
\text { Do not apply thru any type of irrigation } \\
\text { system. }\end{array}$ \\
\hline Lambda-cyhalothrin & Scimitar GC & $F, G$ & $\begin{array}{l}\text { Nursery \& shade house }(\mathrm{F}) \text {; do not apply } \\
\text { more than } 0.36 \text { lbs. a.i. }(52.4 \mathrm{fl} \text {. oz. of } \\
\text { concentrate) per acre per year. }\end{array}$ \\
\hline Malathion & $\begin{array}{l}\text { Atrapa } 5 \mathrm{E} \\
\text { Gowan Malathion } 8 \mathrm{~F} \\
\text { Malathion } 5 \mathrm{EC}\end{array}$ & $\mathrm{F}$ & \\
\hline
\end{tabular}


Table 1. Aphid (Homoptera: Aphididae) control measures available to commercial producers of any flower crop in Florida.

\begin{tabular}{|c|c|c|c|}
\hline Common Name & Trade Name/Formulation & $\begin{array}{l}\text { Production } \\
\text { Site }^{1}\end{array}$ & Notes from Label $^{2}$ \\
\hline Naled & Dibrom 8 Emulsive & $\mathrm{G}$ & $\begin{array}{l}\text { Vapor treatment; spotting, bronzing or } \\
\text { localized burning can occur under certain } \\
\text { climatic conditions such as slow drying and } \\
\text { high humidity or stress caused by drought or } \\
\text { high temperature. White Butterfly and Golden } \\
\text { Rapture roses, Pink Champagne } \\
\text { chrysanthemums, green wandering jew, } \\
\text { poinsettias and Dutchman's pipe may be } \\
\text { injured by vapor. }\end{array}$ \\
\hline Oxydemeton-methyl & Metasystox-R & $\mathrm{F}$ & $\begin{array}{l}\text { Field grown nursery stock; do not make more } \\
\text { than } 2 \text { applications per season. }\end{array}$ \\
\hline
\end{tabular}


Pesticide Options for Important Insect, Mite and Mollusk Pests of Commercial Flowers in....

Table 1. Aphid (Homoptera: Aphididae) control measures available to commercial producers of any flower crop in Florida.

\begin{tabular}{|c|c|c|c|}
\hline Common Name & Trade Name/Formulation & $\begin{array}{l}\text { Production } \\
\text { Site }^{1}\end{array}$ & Notes from Label ${ }^{2}$ \\
\hline Pymetrozine & Endeavor & $F, G$ & $\begin{array}{l}\text { For outdoor use, do not apply more than } 48 \\
\text { oz. per acre per year. For indoor use, do not } \\
\text { apply more than } 100 \mathrm{oz} \text {. per acre per year. }\end{array}$ \\
\hline \multirow{2}{*}{$\begin{array}{l}\text { Pyrethrins \& Piperonyl } \\
\text { Butoxide }\end{array}$} & 1100 Pyrethrum TR & $\mathrm{G}$ & \\
\hline & $\begin{array}{l}\text { Pyrenone Crop Spray } \\
\text { Pyreth-It }\end{array}$ & $F, G$ & \\
\hline $\begin{array}{l}\text { Pyrethrins, Piperonyl } \\
\text { Butoxide \& Silicon } \\
\text { Dioxide }\end{array}$ & $\begin{array}{l}\text { Diatect Multipurpose } \\
\text { Insecticide II }\end{array}$ & $\mathrm{F}$ & \\
\hline $\begin{array}{l}\text { Pyrethrins, Rotenone \& } \\
\text { other Cube Resins }\end{array}$ & Pyrellin E.C. & $F, G$ & Apply in intervals of 7 days or less. \\
\hline Pyriproxyfen & Distance IGR & $F, G$ & $\begin{array}{l}\text { Suppression; for foliar spray application, } \\
\text { apply Distance no more than two times per } \\
\text { cropping cycle or no more than two times per } \\
6 \text { months. For sprench application, if a } \\
\text { second application is needed, allow a } \\
\text { minimum of } 21 \text { days between applications. } \\
\text { For drench application: Do not drench plants } \\
\text { more than one time per crop cycle. } \\
\text { Phytotoxicity has been observed on salvia } \\
\text { (Salvia spp.), ghost plant (Graptopetalum } \\
\text { paraguayense), schefflera (Schefflera spp.), } \\
\text { gardenia (Gardenia spp.), and coral bells } \\
\text { (Heuchera sanguinea). It is therefore } \\
\text { recommended that Distance not be used on } \\
\text { these plants. Do not apply to poinsettia after } \\
\text { bract formation. }\end{array}$ \\
\hline
\end{tabular}


Table 1. Aphid (Homoptera: Aphididae) control measures available to commercial producers of any flower crop in Florida.

\begin{tabular}{|c|c|c|c|}
\hline Common Name & Trade Name/Formulation & $\begin{array}{l}\text { Production } \\
\text { Site }^{1}\end{array}$ & Notes from Label $^{2}$ \\
\hline $\begin{array}{l}\text { Refined Petroleum } \\
\text { Distillate }\end{array}$ & Saf-T-Side Spray Oil & $\mathrm{F}$ & $\begin{array}{l}\text { This insecticide's mode of action is through } \\
\text { suffocation of eggs, larvae and nymphs of } \\
\text { insects and adults of soft bodied insects. This } \\
\text { mode of action necessitates total spray } \\
\text { coverage. Bedding plants; do not use in } \\
\text { combination with or immediately before or } \\
\text { after spraying with fungicides such as captan, } \\
\text { oxythioquinox or any product containing } \\
\text { sulfur. Also do not use with carbaryl (Sevin) } \\
\text { or dimethoate. Do not use with any product } \\
\text { whose label recommends the use of no oils. } \\
\text { Do not use in combination with NPK foliar } \\
\text { fertilizer applications. }\end{array}$ \\
\hline Tau-Fluvalinate & Mavrik Aquaflow & $F, G$ & $\begin{array}{l}\text { Repeat applications not more than four times } \\
\text { per month at } 5-7 \text { day intervals unless } \\
\text { otherwise noted in use directions. }\end{array}$ \\
\hline Thiamethoxam & Flagship 25WG & $F, G$ & $\begin{array}{l}\text { Shade house, container \& nursery grown }(F) \text {; } \\
\text { do not exceed } 8 \text { oz. per acre per crop or year, } \\
\text { which ever is shorter. }\end{array}$ \\
\hline \multicolumn{4}{|c|}{$\begin{array}{l}\text { Footnotes: } \\
1 \text { "F" indicates field production. "G" indicates greenhouse production. } \\
{ }^{2} \text { Notes are taken from product labels and restrict use to the condition indicated (suppression, beet armyworm, exposed } \\
\text { thrips, etc.), limit numbers or patterns of applications, provide phytotoxicity precautions, etc. }\end{array}$} \\
\hline
\end{tabular}


Table 2. Armyworm (Lepidoptera: Noctuidae) control measures available to commercial producers of any flower crop in Florida. Also see Caterpillars (Lepidoptera).

\begin{tabular}{|c|c|c|c|}
\hline Common Name & Trade Name/Formulation & $\begin{array}{l}\text { Production } \\
\text { Site }^{1}\end{array}$ & Notes from Label ${ }^{2}$ \\
\hline Azadirachtin & $\begin{array}{l}\text { Azatin XL } \\
\text { Ornazin 3\% EC }\end{array}$ & $F, G$ & \\
\hline $\begin{array}{l}\text { Bacillus thuringiensis } \\
\text { aizawai }\end{array}$ & Xentari DF & $F, G$ & \\
\hline \multirow[t]{2}{*}{$\begin{array}{l}\text { Bacillus thuringiensis } \\
\text { kurstaki }\end{array}$} & $\begin{array}{l}\text { Dipel DF } \\
\text { Javelin WG }\end{array}$ & $\mathrm{F}, \mathrm{G}$ & \\
\hline & Troy-BT 2WP & $F, G$ & $\begin{array}{l}\text { May be used to control armyworms (1st and } \\
2 \text { nd instars) when populations are light and } \\
\text { full coverage sprays are applied. If mature } \\
\text { worms or heavy populations are present a } \\
\text { contact insecticide should be used to } \\
\text { enhance control. }\end{array}$ \\
\hline $\begin{array}{l}\text { Bacillus thuringiensis } \\
\text { kurstaki strain ABTS-351 }\end{array}$ & Biobit HP & $F, G$ & $\begin{array}{l}\text { May be used to control armyworms (1st and } \\
2 \text { nd instars) when populations are light and } \\
\text { full coverage sprays are applied. If mature } \\
\text { worms or heavy populations are present a } \\
\text { contact insecticide should be used to } \\
\text { enhance control. }\end{array}$ \\
\hline $\begin{array}{l}\text { Bacillus thuringiensis } \\
\text { kurstaki EG2348 }\end{array}$ & Condor Bioinsecticide & $F, G$ & $\begin{array}{l}\text { Precautions: Do not use in combination with } \\
\text { any chlorothalonil based fungicide (Bravo, } \\
\text { Terranil etc.). Mixing with other oil based } \\
\text { products or surfactants could increase the } \\
\text { risk of phytotoxicity. }\end{array}$ \\
\hline $\begin{array}{l}\text { Bacillus thuringiensis } \\
\text { kurstaki EG7841 }\end{array}$ & Crymax Bioinsecticide & $F, G$ & \\
\hline $\begin{array}{l}\text { Beauveria bassiana } \\
\text { ATCC } 74040\end{array}$ & Naturalis L & $F, G$ & $\begin{array}{l}\text { Do not tank mix with fungicides. Wait a } \\
\text { minimum of } 48 \text { hours after application before } \\
\text { applying fungicides. }\end{array}$ \\
\hline \multirow[t]{3}{*}{ Bifenthrin } & $\begin{array}{l}\text { Talstar Flowable } \\
\text { Talstar GH Prescription } \\
\end{array}$ & $\bar{G}$ & Beet armyworm \\
\hline & Talstar N & $F, G$ & $\begin{array}{l}\text { Nursery \& shade house }(F) \text {; beet armyworm } \\
(G) \text {; do not apply more than } 0.2 \text { lbs. a.i. per } \\
\text { acre per year for outdoor applications. }\end{array}$ \\
\hline & Talstar Nursery Flowable & $F, G$ & $\begin{array}{l}\text { Nursery \& shade house }(\mathrm{F}) \text {; do not apply } \\
\text { more than } 0.2 \text { lbs. a.i. per acre per year for } \\
\text { outdoor applications. }\end{array}$ \\
\hline \multirow[t]{3}{*}{ Chlorpyrifos } & Chlorpyrifos Pro 2 & $\mathrm{~F}$ & Nursery stock in nurseries \\
\hline & Chlorpyrifos Pro 4 & $F, G$ & $\begin{array}{l}\text { Nursery }(\mathrm{F}) \text {; soil treatment, dormant spray of } \\
\text { tree pests, and pre-plant incorporation of field } \\
\text { grown nursery stock }(\mathrm{G}) \text {; some varieties of } \\
\text { azaleas, camellias, poinsettias, rose bushes, } \\
\text { or variegated ivy show varying degrees of } \\
\text { phytotoxicity. }\end{array}$ \\
\hline & Dursban 50W & $\mathrm{F}$ & Nursery \\
\hline
\end{tabular}


Table 2. Armyworm (Lepidoptera: Noctuidae) control measures available to commercial producers of any flower crop in Florida. Also see Caterpillars (Lepidoptera).

\begin{tabular}{|c|c|c|c|}
\hline Common Name & Trade Name/Formulation & $\begin{array}{l}\text { Production } \\
\text { Site }^{1}\end{array}$ & Notes from Label ${ }^{2}$ \\
\hline $\begin{array}{l}\text { Capsaicin \& other } \\
\text { capsaicinoids }\end{array}$ & Hot Pepper Wax & $F, G$ & $\begin{array}{l}\text { Repels beet armyworm; avoid use on } \\
\text { variegated African violets and Dicentra } \\
\text { eximia. Do not exceed a rate of } 1: 32 \text { in } \\
\text { greenhouses. Avoid use if greenhouse } \\
\text { temperature exceeds } 85^{\circ} \mathrm{F} \text { before the } \\
\text { product has dried. }\end{array}$ \\
\hline Cyfluthrin & Decathlon $20 \mathrm{WP}$ & $F, G$ & \\
\hline Deltamethrin & Deltagard GC 5 SC & $\mathrm{F}$ & Nursery \\
\hline Diazinon & $\begin{array}{l}\text { Diazinon 50W } \\
\text { Diazinon AG } 500\end{array}$ & $\mathrm{~F}$ & $\begin{array}{l}\text { Precaution: Do not use on ferns, poinsettias, } \\
\text { hibiscus, pilea, and gardenia since injury to } \\
\text { the plants may occur. }\end{array}$ \\
\hline \multirow[t]{3}{*}{ Diflubenzuron } & Adept & $F, G$ & $\begin{array}{l}\text { Shade house }(F) \text {; do not make more than four } \\
\text { applications per crop. Do not apply to } \\
\text { poinsettias, hibiscus, and Rieger begonia. Do } \\
\text { not reuse potting media, which has been } \\
\text { treated with Adept. Do not apply to plants } \\
\text { grown on capillary water mats. }\end{array}$ \\
\hline & Dimilin 25W & $F, G$ & Beet armyworm; shade house (F) \\
\hline & Dimilin SC & $\mathrm{F}, \mathrm{G}$ & Shade house $(F)$ \\
\hline Fenpropathrin & Tame 2.4EC & $F, G$ & $\begin{array}{l}\text { Beet armyworm; nursery \& shade house }(\mathrm{F}) \text {; } \\
\text { do not use less than a } 7 \text { day re-treatment } \\
\text { interval. }\end{array}$ \\
\hline Heterorhabditis indica & GrubStake 100 & $F, G$ & $\begin{array}{l}\text { Store at } 65^{\circ} \mathrm{F} ; \text { use within } 5 \text { days of receipt. } \\
\text { Native to Florida. Do not inject into drip } \\
\text { irrigation systems. Do not apply within } 21 \\
\text { days of a nematicide application. }\end{array}$ \\
\hline Lambda-cyhalothrin & Scimitar GC & $F, G$ & $\begin{array}{l}\text { Nursery \& shade house }(\mathrm{F}) \text {; do not apply } \\
\text { more than } 0.36 \text { lbs. a.i. }(52.4 \mathrm{fl} \text {. oz. of } \\
\text { concentrate) per acre per year. }\end{array}$ \\
\hline Novaluron & Pedestal & $F, G$ & $\begin{array}{l}\text { Container grown plants; nursery \& shade } \\
\text { house (F); do not apply more than once every } \\
30 \text { days. Do not make more than two } \\
\text { applications per crop year. Do not apply more } \\
\text { than } 52 \text { fl. oz. per acre per year per crop. Do } \\
\text { not apply to poinsettias. Resistance } \\
\text { management: Do not apply in successive } \\
\text { applications, use only one application before } \\
\text { rotation with other classes of insecticides with } \\
\text { different modes of action. Use at least two } \\
\text { alternative products between applications of } \\
\text { Pedestal. Do not use more than once within } \\
\text { each generation cycle. }\end{array}$ \\
\hline
\end{tabular}


Table 2. Armyworm (Lepidoptera: Noctuidae) control measures available to commercial producers of any flower crop in Florida. Also see Caterpillars (Lepidoptera).

\begin{tabular}{|c|c|c|c|}
\hline Common Name & Trade Name/Formulation & $\begin{array}{l}\text { Production } \\
\text { Site }^{1}\end{array}$ & Notes from Label ${ }^{2}$ \\
\hline \multirow[t]{5}{*}{ Permethrin } & Ambush 25W & $\bar{F}$ & $\begin{array}{l}\text { Beet armyworm; field grown nursery stock; } \\
\text { do not apply more than } 2.0 \mathrm{lbs} \text {. a.i. per acre } \\
\text { per season. Marginal leaf burn may occur on: } \\
\text { Salvia, dieffenbachia. Application to blooming } \\
\text { plants may cause browning of petals. }\end{array}$ \\
\hline & $\begin{array}{l}\text { Ambush } \\
\text { Pounce 25 WP } \\
\text { Pounce 3.2 EC } \\
\text { Pounce WSB } \\
\end{array}$ & $\mathrm{F}$ & Beet armyworm; field grown nursery stock \\
\hline & Astro & $\bar{G}$ & $\begin{array}{l}\text { Beet armyworm; do not apply more than } 2.0 \\
\text { lb. a.i. per acre per year. }\end{array}$ \\
\hline & $\begin{array}{l}\text { Clean Crop Permethrin } 3.2 \\
\text { EC }\end{array}$ & $\mathrm{F}$ & $\begin{array}{l}\text { Beet armyworm; field grown nursery stock; } \\
\text { marginal leaf burn may occur on: Salvia, } \\
\text { dieffenbachia. Application to blooming plants } \\
\text { may cause browning of petals. Avoid } \\
\text { spraying chrysanthemum blooms. }\end{array}$ \\
\hline & Perm-Up 3.2EC & $\mathrm{F}$ & $\begin{array}{l}\text { Beet armyworm; field grown nursery stock; } \\
\text { marginal leaf burn may occur on: Salvia, } \\
\text { dieffenbachia. }\end{array}$ \\
\hline $\begin{array}{l}\text { Pyrethrins \& Piperonyl } \\
\text { Butoxide }\end{array}$ & $\begin{array}{l}\text { Pyrenone Crop Spray } \\
\text { Pyreth-It }\end{array}$ & $\mathrm{F}, \mathrm{G}$ & \\
\hline $\begin{array}{l}\text { Pyrethrins, Rotenone \& } \\
\text { other Cube Resins }\end{array}$ & Pyrellin E.C. & $\mathrm{F}, \mathrm{G}$ & Apply in intervals of 7 days or less. \\
\hline Spinosad & Conserve SC & $F, G$ & $\begin{array}{l}\text { When used in greenhouse or areas of } \\
\text { commercial production of herbaceous } \\
\text { (non-woody) ornamentals in nurseries } \\
\text { (including plant propagation beds): Do not } \\
\text { apply more than } 10 \text { times in a 12-month } \\
\text { period per crop regardless of the pest being } \\
\text { treated. Because generations of a specific } \\
\text { pest may overlap, rotate control products and } \\
\text { never apply more than three consecutive } \\
\text { applications of Conserve SC or products } \\
\text { containing the same a.i. or products with the } \\
\text { same mode of action. Except for } \\
\text { greenhouses and structures that can be } \\
\text { altered to be closed or open, do not reapply } \\
\text { within less than } 7 \text { days. }\end{array}$ \\
\hline $\begin{array}{l}\text { Steinernema } \\
\text { carpocapsae }\end{array}$ & Millenium & $F, G$ & Nursery (F) \\
\hline Tau-Fluvalinate & Mavrik Aquaflow & $F, G$ & $\begin{array}{l}\text { Repeat applications not more than four times } \\
\text { per month at 5-7 day intervals unless } \\
\text { otherwise noted in use directions. }\end{array}$ \\
\hline
\end{tabular}


Table 2. Armyworm (Lepidoptera: Noctuidae) control measures available to commercial producers of any flower crop in Florida. Also see Caterpillars (Lepidoptera).

\begin{tabular}{|c|c|c|c|}
\hline Common Name & Trade Name/Formulation & $\begin{array}{l}\text { Production } \\
\text { Site }^{1}\end{array}$ & Notes from Label $^{2}$ \\
\hline Tebufenozide & Confirm T/O & $F, G$ & $\begin{array}{l}\text { Nursery }(F) \text {; do not use to control more than } \\
\text { three consecutive generations of pests in a } \\
\text { cropping area (field) regardless of the crop } \\
\text { rotation on that field. If you are unsure of the } \\
\text { number of generations treated do not use } \\
\text { more than four times within any } 80 \text {-day } \\
\text { interval. If Confirm has been applied four } \\
\text { times in } 80 \text { days or less, allow at least } 40 \\
\text { days to pass before making additional } \\
\text { applications. }\end{array}$ \\
\hline \multicolumn{4}{|c|}{$\begin{array}{l}\text { Footnotes: } \\
1 \text { "F" indicates field production. "G" indicates greenhouse production. } \\
{ }^{2} \text { Notes are taken from product labels and restrict use to the condition indicated (suppression, beet armyworm, exposed } \\
\text { thrips, etc.), limit numbers or patterns of applications, provide phytotoxicity precautions, etc. }\end{array}$} \\
\hline
\end{tabular}

Table 3. Control measures for other caterpillars (Lepidoptera) available to commercial producers of any flower crop in Florida. Also see tables for these other named caterpillars: Armyworms, Helicoverpa (corn earworm, etc) and Heliothis (tobacco budworm, etc.) Leafrollers, Leaftiers, and Loopers.

\begin{tabular}{|c|c|c|c|}
\hline Common Name & Trade Name/Formulation & $\begin{array}{l}\text { Production } \\
\text { Site }^{1}\end{array}$ & Notes from Label $^{2}$ \\
\hline Azadirachtin & $\begin{array}{l}\text { Azatin XL } \\
\text { Ornazin } 3 \% \text { EC }\end{array}$ & $\mathrm{F}, \mathrm{G}$ & \\
\hline Acephate & 1300 Orthene TR & $G$ & Tent caterpillars \\
\hline $\begin{array}{l}\text { Acephate \& } \\
\text { Fenpropathrin }\end{array}$ & Tame/Orthene TR & $\mathrm{G}$ & $\begin{array}{l}\text { Tent caterpillars; do not apply within } 48 \text { hours } \\
\text { of a previous application. }\end{array}$ \\
\hline \multirow[t]{3}{*}{$\begin{array}{l}\text { Bacillus thuringiensis } \\
\text { kurstaki }\end{array}$} & Dipel DF & $F, G$ & $\begin{array}{l}\text { Azalea caterpillar, bagworm, diamondback } \\
\text { moth, ello moth (hornworm), Fall webworm, io } \\
\text { moth, oleander moth, saltmarsh caterpillar, } \\
\text { tent caterpillars }\end{array}$ \\
\hline & Javelin WG & $F, G$ & $\begin{array}{l}\text { Bagworm, bollworm, cutworm, diamondback } \\
\text { moth, Fall webworm, filbert webworm, } \\
\text { hornworms, orangedog, saltmarsh caterpillar, } \\
\text { tent caterpillar, tobacco hornworm, tomato } \\
\text { pinworm }\end{array}$ \\
\hline & Troy-BT 2WP & $F, G$ & $\begin{array}{l}\text { Azalea caterpillar, diamondback moth, ello } \\
\text { moth (hornworm), io moth, oleander moth }\end{array}$ \\
\hline $\begin{array}{l}\text { Bacillus thuringiensis } \\
\text { kurstaki strain ABTS-351 }\end{array}$ & Biobit HP & $F, G$ & $\begin{array}{l}\text { Azalea caterpillar, diamondback moth, ello } \\
\text { moth (hornworm), io moth, oleander moth, } \\
\text { sod webworm }\end{array}$ \\
\hline
\end{tabular}


Table 3. Control measures for other caterpillars (Lepidoptera) available to commercial producers of any flower crop in Florida. Also see tables for these other named caterpillars: Armyworms, Helicoverpa (corn earworm, etc) and Heliothis (tobacco budworm, etc.) Leafrollers, Leaftiers, and Loopers.

\begin{tabular}{|c|c|c|c|}
\hline Common Name & Trade Name/Formulation & $\begin{array}{l}\text { Production } \\
\text { Site }^{1}\end{array}$ & Notes from Label $^{2}$ \\
\hline $\begin{array}{l}\text { Bacillus thuringiensis } \\
\text { kurstaki EG2348 }\end{array}$ & Condor Bioinsecticide & $F, G$ & $\begin{array}{l}\text { Azalea moth, bagworm }(F) \text {, diamondback } \\
\text { moth, ello moth (hornworm), fall webworm } \\
(F) \text {, io moth, oleander moth, saddleback } \\
\text { caterpillar }(F) \text {, tent caterpillar }(F) \text {, tortrix }(F) \text {; } \\
\text { precautions: Do not use in combination with } \\
\text { any chlorothalonil based fungicide (Bravo, } \\
\text { Terranil etc.). Mixing with other oil based } \\
\text { products or surfactants could increase the } \\
\text { risk of phytotoxicity. }\end{array}$ \\
\hline $\begin{array}{l}\text { Bacillus thuringiensis } \\
\text { kurstaki EG7841 }\end{array}$ & Crymax Bioinsecticide & $F, G$ & $\begin{array}{l}\text { Azalea moth, bagworm (F), diamondback } \\
\text { moth, ello moth (hornworm), fall webworm, } \\
\text { Florida fern caterpillar, io moth, oleander } \\
\text { moth, saddleback caterpillar (F), tent } \\
\text { caterpillar (F), tortrix (F) }\end{array}$ \\
\hline $\begin{array}{l}\text { Beauveria bassiana } \\
\text { ATCC } 74040\end{array}$ & Naturalis L & $F, G$ & $\begin{array}{l}\text { Do not tank mix with fungicides. Wait a } \\
\text { minimum of } 48 \text { hours after application before } \\
\text { applying fungicides. }\end{array}$ \\
\hline Bifenthrin & $\begin{array}{l}\text { Talstar N } \\
\text { Talstar Nursery Flowable }\end{array}$ & $F, G$ & $\begin{array}{l}\text { Nursery \& shade house (F); do not apply } \\
\text { more than } 0.2 \text { lbs. a.i. per acre per year for } \\
\text { outdoor applications. }\end{array}$ \\
\hline \multirow[t]{3}{*}{ Carbaryl } & $\begin{array}{l}\text { Carbaryl 4L } \\
\text { Carbaryl 80S }\end{array}$ & $\mathrm{F}$ & $\begin{array}{l}\text { Bagworms, catalpa sphinx, cutworms, } \\
\text { oleander caterpillar, poinsettia hornworm, } \\
\text { puss caterpillar, tent caterpillars, webworms }\end{array}$ \\
\hline & Cutworm \& Cricket Bait & $\mathrm{F}$ & Cutworm \\
\hline & $\begin{array}{l}\text { Sevin } 80 \text { WSP } \\
\text { Sevin SL }\end{array}$ & $\bar{F}$ & $\begin{array}{l}\text { Bagworms, catalpa sphinx, cutworms, } \\
\text { oleander caterpillar, poinsettia hornworm, } \\
\text { puss caterpillar, tent caterpillars, webworms; } \\
\text { application to wet foliage or during periods of } \\
\text { high humidity may cause injury to tender } \\
\text { foliage. }\end{array}$ \\
\hline Chlorfenapyr & Pylon & G & $\begin{array}{l}\text { Non-edible ornamentals; do not apply Pylon } \\
\text { more than two times consecutively or a total } \\
\text { of three times during a growing cycle (not } \\
\text { more than } 41 \mathrm{fl} \text {. oz. of product/100 gal. / crop } \\
\text { / season or } 0.64 \text { lbs. of a.i. / } 100 \text { gal. / crop / } \\
\text { season) (start to finish for one ornamental } \\
\text { crop). Do not apply Pylon to consecutive } \\
\text { crops in a greenhouse unless the Pylon is } \\
\text { applied in combination with other effective } \\
\text { miticides or insecticides with a different mode } \\
\text { of action. Phytotoxicity is likely to occur to } \\
\text { some varieties of carnations, dianthus, } \\
\text { kalanchoe, poinsettia, roses, salvia, and } \\
\text { zinnia. Additions of crop oils, surfactants, and } \\
\text { fertilizers or other tank additives have been } \\
\text { shown to increase the likelihood of foliar } \\
\text { injury. }\end{array}$ \\
\hline
\end{tabular}


Table 3. Control measures for other caterpillars (Lepidoptera) available to commercial producers of any flower crop in Florida. Also see tables for these other named caterpillars: Armyworms, Helicoverpa (corn earworm, etc) and Heliothis (tobacco budworm, etc.) Leafrollers, Leaftiers, and Loopers.

\begin{tabular}{|c|c|c|c|}
\hline Common Name & Trade Name/Formulation & $\begin{array}{l}\text { Production } \\
\text { Site }^{1}\end{array}$ & Notes from Label ${ }^{2}$ \\
\hline \multirow[t]{4}{*}{ Chlorpyrifos } & Chlorpyrifos Pro 2 & $\mathrm{~F}$ & $\begin{array}{l}\text { Bagworms, catalpa sphinx, cutworms, Fall } \\
\text { webworms, hornworms, oleander caterpillars, } \\
\text { puss caterpillars, tent caterpillars; nursery } \\
\text { stock in nurseries }\end{array}$ \\
\hline & Chlorpyrifos Pro 4 & $F, G$ & $\begin{array}{l}\text { Bagworms, catalpa sphinx, cutworms, Fall } \\
\text { webworms, hornworms, oleander caterpillars, } \\
\text { puss caterpillars, tent caterpillars; nursery } \\
\text { (F); soil treatment, dormant spray of tree } \\
\text { pests, and pre-plant incorporation of field } \\
\text { grown nursery stock (G); some varieties of } \\
\text { azaleas, camellias, poinsettias, rose bushes, } \\
\text { or variegated ivy show varying degrees of } \\
\text { phytotoxicity. }\end{array}$ \\
\hline & Duragard ME & $F, G$ & $\begin{array}{l}\text { Cabbage worms, hornworms; nursery }(\mathrm{F}) \text {; } \\
\text { bedding plants, potted and cut flowers. Direct } \\
\text { spray to some open blooms may cause petal } \\
\text { drop. Do not spray on kalanchoes. }\end{array}$ \\
\hline & Dursban 50W & $\mathrm{F}$ & $\begin{array}{l}\text { Bagworms, catalpa sphinx, cutworms, Fall } \\
\text { webworms, hornworms, oleander } \\
\text { caterpillars, puss caterpillars, tent } \\
\text { caterpillars; nursery }\end{array}$ \\
\hline Cyfluthrin & Decathlon 20 WP & F. G & \\
\hline Deltamethrin & Deltagard GC 5 SC & $\mathrm{F}$ & Nursery \\
\hline Diazinon & $\begin{array}{l}\text { Diazinon 50W } \\
\text { Diazinon AG } 500\end{array}$ & $\mathrm{~F}$ & $\begin{array}{l}\text { Bagworms, cutworms, Fall webworms, tent } \\
\text { caterpillars; precaution: Do not use on ferns, } \\
\text { poinsettias, hibiscus, pilea, and gardenia } \\
\text { since injury to the plants may occur. }\end{array}$ \\
\hline Heterorhabditis indica & GrubStake 100 & $F, G$ & $\begin{array}{l}\text { Webworm, tree borers including banana } \\
\text { moth; store at } 65^{\circ} \mathrm{F} ; \text { use within } 5 \text { days of } \\
\text { receipt. Native to Florida. Do not inject into } \\
\text { drip irrigation systems. Do not apply within } 21 \\
\text { days of a nematicide application. }\end{array}$ \\
\hline Lambda-cyhalothrin & Scimitar GC & $F, G$ & $\begin{array}{l}\text { Leaf-feeding caterpillars \& cutworms; nursery } \\
\& \text { shade house }(F) \text {; do not apply more than } \\
0.36 \text { lbs. a.i. ( } 52.4 \text { fl. oz. of concentrate) per } \\
\text { acre per year. }\end{array}$ \\
\hline Malathion & $\begin{array}{l}\text { Atrapa } 5 \mathrm{E} \\
\text { Gowan Malathion } 8 \mathrm{~F} \\
\text { Malathion } 5 \\
\text { Malathion } 5 \mathrm{EC}\end{array}$ & $\mathrm{F}$ & Bagworm, tent caterpillar \\
\hline
\end{tabular}


Table 3. Control measures for other caterpillars (Lepidoptera) available to commercial producers of any flower crop in Florida. Also see tables for these other named caterpillars: Armyworms, Helicoverpa (corn earworm, etc) and Heliothis (tobacco budworm, etc.) Leafrollers, Leaftiers, and Loopers.

\begin{tabular}{|c|c|c|c|}
\hline Common Name & Trade Name/Formulation & $\begin{array}{l}\text { Production } \\
\text { Site }^{1}\end{array}$ & Notes from Label $^{2}$ \\
\hline \multirow[t]{5}{*}{ Permethrin } & Ambush 25W & $\mathrm{F}$ & $\begin{array}{l}\text { Cutworms; field grown nursery stock; do not } \\
\text { apply more than } 2.0 \mathrm{lbs} \text {. a.i. per acre per } \\
\text { season. Marginal leaf burn may occur on: } \\
\text { Salvia, dieffenbachia. Application to blooming } \\
\text { plants may cause browning of petals. }\end{array}$ \\
\hline & Ambush & $\mathrm{F}$ & Cutworm; field grown nursery stock \\
\hline & $\begin{array}{l}\text { Pounce } 25 \text { WP } \\
\text { Pounce } 3.2 \text { EC } \\
\text { Pounce WSB }\end{array}$ & $\mathrm{F}$ & Bagworm; field grown nursery stock \\
\hline & $\begin{array}{l}\text { Clean Crop Permethrin } 3.2 \\
\text { EC }\end{array}$ & $\mathrm{F}$ & $\begin{array}{l}\text { Bagworm; field grown nursery stock; } \\
\text { marginal leaf burn may occur on: Salvia, } \\
\text { dieffenbachia. Application to blooming plants } \\
\text { may cause browning of petals. Avoid } \\
\text { spraying chrysanthemum blooms. }\end{array}$ \\
\hline & Perm-Up 3.2 EC & $\mathrm{F}$ & $\begin{array}{l}\text { Bagworm; field grown nursery stock; } \\
\text { marginal leaf burn may occur on: Salvia, } \\
\text { dieffenbachia. }\end{array}$ \\
\hline
\end{tabular}


Table 3. Control measures for other caterpillars (Lepidoptera) available to commercial producers of any flower crop in Florida. Also see tables for these other named caterpillars: Armyworms, Helicoverpa (corn earworm, etc) and Heliothis (tobacco budworm, etc.) Leafrollers, Leaftiers, and Loopers.

\begin{tabular}{|c|c|c|c|}
\hline Common Name & Trade Name/Formulation & $\begin{array}{l}\text { Production } \\
\text { Site }^{1}\end{array}$ & Notes from Label ${ }^{2}$ \\
\hline \multirow[t]{2}{*}{$\begin{array}{l}\text { Potassium Salts of Fatty } \\
\text { Acids }\end{array}$} & Insecticidal Soap $49.52 \mathrm{CF}$ & $\mathrm{F}, \mathrm{G}$ & $\begin{array}{l}\text { Tent caterpillars; caution: Do not use on new } \\
\text { transplants, newly rooted cuttings, plants } \\
\text { stressed by drought, or when tender new } \\
\text { foliage is present. Avoid application when } \\
\text { leaf temperature exceeds } 85^{\circ} \text { F. Note: Do } \\
\text { not use on crown of thorns and test on other } \\
\text { euphorbias for phytotoxicity. Do not use on } \\
\text { bleeding heart, lantana, lilies or sweetpeas. } \\
\text { Some varieties of azaleas, begonias, } \\
\text { camellias, fuschias, gardenias, orchids, and } \\
\text { impatiens have shown sensitivity. Do not } \\
\text { apply to poinsettias after bract coloration } \\
\text { begins. Test on chrysanthemums for varietal } \\
\text { sensitivity and do not apply to open blooms. } \\
\text { Do not apply to ornamentals stressed by } \\
\text { drought. Test on eugenia and euonymus for } \\
\text { varietal and drought sensitivity. }\end{array}$ \\
\hline & M-Pede & $F, G$ & $\begin{array}{l}\text { Buck moth larvae, cutworm (F), tent } \\
\text { caterpillar. Tank mixes with chlorothalonil } \\
\text { should be avoided. Potential for injury } \\
\text { increases on plants stressed by heat, } \\
\text { humidity, drought, or insect, mite or disease } \\
\text { pressure. Do not apply to very sensitive } \\
\text { plants such as: Cherimoya, bleeding heart, or } \\
\text { sweetpeas. The following may be sensitive: } \\
\text { Crown of thorns and other euphorbia, fuchsia, } \\
\text { gardenia, impatiens, Asiatic and oriental lily } \\
\text { varieties, lantana, ornamental ivy, poinsettia, } \\
\text { redbud, schefflera, zebra plant and some } \\
\text { succulents. Flowers of the following may be } \\
\text { injured when sprayed: African violet, } \\
\text { ageratum, azalea, begonia, camellia, } \\
\text { chrysanthemum, dahlia, geranium, gloxinia, } \\
\text { impatiens, lily, marigold, orchid, pansy, } \\
\text { petunia, poinsettia bracts, rose, salvia, } \\
\text { snapdragon, vinca, and zinnia. }\end{array}$ \\
\hline $\begin{array}{l}\text { Pyrethrins \& Piperonyl } \\
\text { Butoxide }\end{array}$ & $\begin{array}{l}\text { Pyrenone Crop Spray } \\
\text { Pyreth-It }\end{array}$ & F. G & \\
\hline $\begin{array}{l}\text { Pyrethrins, Piperonyl } \\
\text { Butoxide \& Silicon } \\
\text { Dioxide }\end{array}$ & $\begin{array}{l}\text { Diatect Multipurpose } \\
\text { Insecticide II }\end{array}$ & $\mathrm{F}$ & \\
\hline $\begin{array}{l}\text { Pyrethrins, Rotenone \& } \\
\text { other Cube Resins }\end{array}$ & Pyrellin E.C. & $F, G$ & Apply in intervals of 7 days or less. \\
\hline
\end{tabular}


Table 3. Control measures for other caterpillars (Lepidoptera) available to commercial producers of any flower crop in Florida. Also see tables for these other named caterpillars: Armyworms, Helicoverpa (corn earworm, etc) and Heliothis (tobacco budworm, etc.) Leafrollers, Leaftiers, and Loopers.

\begin{tabular}{|c|c|c|c|}
\hline Common Name & Trade Name/Formulation & $\begin{array}{l}\text { Production } \\
\text { Site }^{1}\end{array}$ & Notes from Label ${ }^{2}$ \\
\hline \multirow[t]{2}{*}{$\begin{array}{l}\text { Refined Petroleum } \\
\text { Distillate }\end{array}$} & Saf-T-Side Spray Oil & $\mathrm{F}$ & $\begin{array}{l}\text { This insecticide's mode of action is through } \\
\text { suffocation of eggs, larvae and nymphs of } \\
\text { insects and adults of soft bodied insects. This } \\
\text { mode of action necessitates total spray } \\
\text { coverage. Webworms; bedding plants; do not } \\
\text { use in combination with or immediately } \\
\text { before or after spraying with fungicides such } \\
\text { as captan, oxythioquinox or any product } \\
\text { containing sulfur. Also do not use with } \\
\text { carbaryl (Sevin) or dimethoate. Do not use } \\
\text { with any product whose label recommends } \\
\text { the use of no oils. Do not use in combination } \\
\text { with NPK foliar fertilizer applications. }\end{array}$ \\
\hline & Ultra-Fine Oil & $\mathrm{F}$ & $\begin{array}{l}\text { Webworms; caution: Spray no more than } 4 \\
\text { consecutive sprays. The frequency of } \\
\text { consecutive sprays should not exceed once } \\
\text { every } 2 \text { weeks. Early morning applications } \\
\text { are recommended. Do not tank mix with } \\
\text { insecticide or miticide whose label indicates } \\
\text { that it should not be used with oil. Do not use } \\
\text { with captan, chlorothalonil, dimethoate, } \\
\text { methiocarb, oxythioquinox, propargite, or any } \\
\text { product containing sulfur. This list is not } \\
\text { exhaustive; therefore for products not } \\
\text { included in this list consult label for } \\
\text { compatibility information. }\end{array}$ \\
\hline Spinosad & Conserve SC & $F, G$ & $\begin{array}{l}\text { Lepidopterous larvae; when used in } \\
\text { greenhouse or areas of commercial } \\
\text { production of herbaceous (non-woody) } \\
\text { ornamentals in nurseries (including plant } \\
\text { propagation beds): Do not apply more than } \\
10 \text { times in a 12-month period per crop } \\
\text { regardless of the pest being treated. Because } \\
\text { generations of a specific pest may overlap, } \\
\text { rotate control products and never apply more } \\
\text { than three consecutive applications of } \\
\text { Conserve SC or products containing the } \\
\text { same a.i. or products with the same mode of } \\
\text { action. Except for greenhouses and } \\
\text { structures that can be altered to be closed or } \\
\text { open, do not reapply within less than } 7 \text { days. }\end{array}$ \\
\hline $\begin{array}{l}\text { Steinernema } \\
\text { carpocapsae }\end{array}$ & Millenium & $F, G$ & $\begin{array}{l}\text { Nursery (F); cutworms, sod webworms, } \\
\text { banana moths }\end{array}$ \\
\hline Tau-Fluvalinate & Mavrik Aquaflow & $F, G$ & $\begin{array}{l}\text { Cutworms, leaf-feeding caterpillars; repeat } \\
\text { applications not more than four times per } \\
\text { month at 5-7 day intervals unless otherwise } \\
\text { noted in use directions. }\end{array}$ \\
\hline
\end{tabular}


Table 3. Control measures for other caterpillars (Lepidoptera) available to commercial producers of any flower crop in Florida. Also see tables for these other named caterpillars: Armyworms, Helicoverpa (corn earworm, etc) and Heliothis (tobacco budworm, etc.) Leafrollers, Leaftiers, and Loopers.

\begin{tabular}{||l|l|l|l||}
\hline \hline \multicolumn{1}{|c|}{ Common Name } & Trade Name/Formulation & $\begin{array}{c}\text { Production } \\
\text { Site }\end{array}$ & \multicolumn{1}{|c||}{ Notes from Label $^{2}$} \\
\hline Tebufenozide & Confirm T/O & F, G & $\begin{array}{l}\text { Bagworms, cutworms, fall webworm, Florida } \\
\text { fern caterpillar, puss caterpillar, Eastern tent } \\
\text { caterpillar, Forest tent caterpillar, Western } \\
\text { tent caterpillar; nursery (F); do not use to } \\
\text { control more than three consecutive } \\
\text { generations of pests in a cropping area (field) } \\
\text { regardless of the crop rotation on that field. If } \\
\text { you are unsure of the number of generations } \\
\text { treated do not use more than four times } \\
\text { within any 80-day interval. If Confirm has } \\
\text { been applied four times in 80 days or less, } \\
\text { allow at least 40 days to pass before making } \\
\text { additional applications. }\end{array}$ \\
\hline $\begin{array}{l}\text { Footnotes: } \\
1 \text { "F" indicates field production. "G" indicates greenhouse production. } \\
2 \text { Notes are taken from product labels and restrict use to the condition indicated (suppression, beet armyworm, exposed } \\
\text { thrips, etc.), limit numbers or patterns of applications, provide phytotoxicity precautions, etc. }\end{array}$ \\
\hline
\end{tabular}

Table 4. Chrysomelid Beetle (cucumber, flea, and leaf beetles) (Coleoptera: Chrysomelidae) control measures available to commercial producers of any flower crop in Florida.

\begin{tabular}{|c|c|c|c|}
\hline Common Name & Trade Name/Formulation & $\begin{array}{l}\text { Production } \\
\text { Site }^{1}\end{array}$ & Notes from Label ${ }^{2}$ \\
\hline \multirow[t]{2}{*}{ Azadirachtin } & Azatin XL & $F, G$ & \\
\hline & Ornazin 3\% EC & $\mathrm{F}$ & \\
\hline $\begin{array}{l}\text { Beauveria bassiana } \\
\text { ATCC } 74040\end{array}$ & Naturalis L & $F, G$ & $\begin{array}{l}\text { Cucumber \& flea beetles; do not tank mix with } \\
\text { fungicides. Wait a minimum of } 48 \text { hours after } \\
\text { application before applying fungicides. }\end{array}$ \\
\hline \multirow[t]{2}{*}{ Bifenthrin } & $\begin{array}{l}\text { Talstar GH Prescription } \\
\text { Talstar Flowable }\end{array}$ & $\mathrm{G}$ & Flea beetles \\
\hline & $\begin{array}{l}\text { Talstar N } \\
\text { Talstar Nursery Flowable }\end{array}$ & $F, G$ & $\begin{array}{l}\text { Flea beetles; nursery \& shade house }(F) \text {; do } \\
\text { not apply more than } 0.2 \text { lbs. a.i. per acre per } \\
\text { year for outdoor applications. }\end{array}$ \\
\hline \multirow[t]{2}{*}{ Carbaryl } & $\begin{array}{l}\text { Carbaryl 4L } \\
\text { Carbaryl 80S } \\
\end{array}$ & $\mathrm{F}$ & Flea beetle \\
\hline & $\begin{array}{l}\text { Sevin } 80 \text { WSP } \\
\text { Sevin SL }\end{array}$ & $\mathrm{F}$ & $\begin{array}{l}\text { Flea beetle; application to wet foliage or } \\
\text { during periods of high humidity may cause } \\
\text { injury to tender foliage. }\end{array}$ \\
\hline
\end{tabular}


Table 4. Chrysomelid Beetle (cucumber, flea, and leaf beetles) (Coleoptera: Chrysomelidae) control measures available to commercial producers of any flower crop in Florida.

\begin{tabular}{|c|c|c|c|}
\hline Common Name & Trade Name/Formulation & $\begin{array}{l}\text { Production } \\
\text { Site }^{1}\end{array}$ & Notes from Label ${ }^{2}$ \\
\hline \multirow[t]{4}{*}{ Chlorpyrifos } & Chlorpyrifos Pro 2 & $\mathrm{~F}$ & Nursery stock in inurseries \\
\hline & Chlorpyrifos Pro 4 & $F, G$ & $\begin{array}{l}\text { Nursery }(\mathrm{F}) \text {; soil treatment, dormant spray of } \\
\text { tree pests, and pre-plant incorporation of field } \\
\text { grown nursery stock }(\mathrm{G}) \text {; some varieties of } \\
\text { azaleas, camellias, poinsettias, rose bushes, } \\
\text { or variegated ivy show varying degrees of } \\
\text { phytotoxicity. }\end{array}$ \\
\hline & Duragard ME & $F, G$ & $\begin{array}{l}\text { Nursery (F); bedding plants, potted and cut } \\
\text { flowers. Direct spray to some open blooms } \\
\text { may cause petal drop. Do not spray on } \\
\text { kalanchoes. }\end{array}$ \\
\hline & Dursban 50W & $\mathrm{F}$ & Nursery \\
\hline Cryolite & Kryocide & $\mathrm{F}$ & Flea beetles \\
\hline Cyfluthrin & Decathlon 20WP & $\mathrm{F}, \mathrm{G}$ & Flea beetles \\
\hline Deltamethrin & Deltagard GC 5 SC & $\mathrm{F}$ & Flea beetles; nursery \\
\hline Diazinon & $\begin{array}{l}\text { Diazinon 50W } \\
\text { Diazinon AG } 500\end{array}$ & $\mathrm{~F}$ & $\begin{array}{l}\text { Flea beetles; precaution: Do not use on } \\
\text { ferns, poinsettias, hibiscus, pilea, and } \\
\text { gardenia since injury to the plants may } \\
\text { occur. }\end{array}$ \\
\hline Imidacloprid & Marathon II & $F, G$ & $\begin{array}{l}\text { Leaf beetles; for outdoor ornamentals do not } \\
\text { apply more than } 1.6 \text { pt. ( } 0.4 \mathrm{lbs} \text {. a.i.) per acre } \\
\text { per year. Do not apply thru any type of } \\
\text { irrigation system. }\end{array}$ \\
\hline Lambda-cyhalothrin & Scimitar GC & $F, G$ & $\begin{array}{l}\text { Flea beetles; nursery \& shade house (F); do } \\
\text { not apply more than } 0.36 \text { lbs. a.i. ( } 52.4 \mathrm{fl} \text {. oz. } \\
\text { of concentrate) per acre per year. }\end{array}$ \\
\hline \multirow{3}{*}{$\begin{array}{l}\text { Pyrethrins \& Piperonyl } \\
\text { Butoxide }\end{array}$} & 1100 Pyrethrum TR & $\mathrm{G}$ & Flea, cucumber \& watermelon beetles \\
\hline & Pyrenone Crop Spray & $F, G$ & \\
\hline & Pyreth-It & $\mathrm{F}, \mathrm{G}$ & Flea \& cucumber beetles \\
\hline $\begin{array}{l}\text { Pyrethrins, Piperonyl } \\
\text { Butoxide \& Silicon } \\
\text { Dioxide }\end{array}$ & $\begin{array}{l}\text { Diatect Multipurpose } \\
\text { Insecticide II }\end{array}$ & $\mathrm{F}$ & Flea beetles \\
\hline $\begin{array}{l}\text { Refined Petroleum } \\
\text { Distillate }\end{array}$ & Ultra-Fine Oil & $\mathrm{F}$ & $\begin{array}{l}\text { Leaf beetle larvae; caution: Spray no more } \\
\text { than } 4 \text { consecutive sprays. The frequency of } \\
\text { consecutive sprays should not exceed once } \\
\text { every } 2 \text { weeks. Early morning applications } \\
\text { are recommended. Do not tank mix with } \\
\text { insecticide or miticide whose label indicates } \\
\text { that it should not be used with oil. Do not use } \\
\text { with captan, chlorothalonil, dimethoate, } \\
\text { methiocarb, oxythioquinox, propargite, or any } \\
\text { product containing sulfur. This list is not } \\
\text { exhaustive; therefore for products not } \\
\text { included in this list consult label for } \\
\text { compatibility information. }\end{array}$ \\
\hline
\end{tabular}


Table 4. Chrysomelid Beetle (cucumber, flea, and leaf beetles) (Coleoptera: Chrysomelidae) control measures available to commercial producers of any flower crop in Florida.

\begin{tabular}{|c|c|c|c|}
\hline Common Name & Trade Name/Formulation & $\begin{array}{l}\text { Production } \\
\text { Site }^{1}\end{array}$ & Notes from Label ${ }^{2}$ \\
\hline Spinosad & Conserve SC & $F, G$ & $\begin{array}{l}\text { When used in greenhouse or areas of } \\
\text { commercial production of herbaceous } \\
\text { (non-woody) ornamentals in nurseries } \\
\text { (including plant propagation beds): Do not } \\
\text { apply more than } 10 \text { times in a 12-month } \\
\text { period per crop regardless of the pest being } \\
\text { treated. Because generations of a specific } \\
\text { pest may overlap, rotate control products and } \\
\text { never apply more than three consecutive } \\
\text { applications of Conserve SC or products } \\
\text { containing the same a.i. or products with the } \\
\text { same mode of action. Except for } \\
\text { greenhouses and structures that can be } \\
\text { altered to be closed or open, do not reapply } \\
\text { within less than } 7 \text { days. }\end{array}$ \\
\hline Tau-Fluvalinate & Mavrik Aquaflow & $F, G$ & $\begin{array}{l}\text { Flea \& cucumber beetles; repeat applications } \\
\text { not more than four times per month at } 5-7 \\
\text { day intervals unless otherwise noted in use } \\
\text { directions. }\end{array}$ \\
\hline \multicolumn{4}{|c|}{$\begin{array}{l}\text { Footnotes: } \\
1 \text { "F" indicates field production. "G" indicates greenhouse production. } \\
2 \text { Notes are taken from product labels and restrict use to the condition indicated (suppression, beet armyworm, exposed } \\
\text { thrips, etc.), limit numbers or patterns of applications, provide phytotoxicity precautions, etc. }\end{array}$} \\
\hline
\end{tabular}

Table 5. Fleahopper (Hemiptera: Miridae) control measures available to commercial producers of any flower crop in Florida.

\begin{tabular}{|c|c|c|c|}
\hline Common Name & Trade Name/Formulation & $\begin{array}{l}\text { Production } \\
\text { Site }^{1}\end{array}$ & Notes from Label $^{2}$ \\
\hline $\begin{array}{l}\text { Pyrethrins, Piperonyl } \\
\text { Butoxide \& Silicon } \\
\text { Dioxide }\end{array}$ & $\begin{array}{l}\text { Diatect Multipurpose } \\
\text { Insecticide II }\end{array}$ & $\mathrm{F}$ & \\
\hline $\begin{array}{l}\text { Pyrethrins, Rotenone \& } \\
\text { other Cube Resins }\end{array}$ & Pyrellin E.C. & $F, G$ & Apply in intervals of 7 days or less. \\
\hline \multicolumn{4}{|c|}{$\begin{array}{l}\text { Footnotes: } \\
1 \text { "F" indicates field production. "G" indicates greenhouse production. } \\
{ }^{2} \text { Notes are taken from product labels and restrict use to the condition indicated (suppression, beet armyworm, exposed } \\
\text { thrips, etc.), limit numbers or patterns of applications, provide phytotoxicity precautions, etc. }\end{array}$} \\
\hline
\end{tabular}


Table 6. Fungus Gnat (Diptera: Sciaridae) control measures available to commercial producers of any flower crop in Florida.

\begin{tabular}{|c|c|c|c|}
\hline Common Name & Trade Name/Formulation & $\begin{array}{l}\text { Production } \\
\text { Site }^{1}\end{array}$ & Notes from Label ${ }^{2}$ \\
\hline Acephate & 1300 Orthene TR & $G$ & \\
\hline Acephate \& Fenpropathrin & Tame/Orthene TR & $G$ & $\begin{array}{l}\text { Do not apply within } 48 \text { hours of a previous } \\
\text { application. }\end{array}$ \\
\hline Azadirachtin & $\begin{array}{l}\text { Azatin XL } \\
\text { Ornazin } 3 \% \text { EC }\end{array}$ & $F, G$ & \\
\hline $\begin{array}{l}\text { Bacillus thuringiensis } \\
\text { israelensis }\end{array}$ & Gnatrol & $F, G$ & $\begin{array}{l}\text { In potting soil mixtures only }(\mathrm{F}) \text {; soil drench } \\
\text { for control of larvae. }\end{array}$ \\
\hline $\begin{array}{l}\text { Beauveria bassiana } \\
\text { ATCC } 74040\end{array}$ & Naturalis L & $F, G$ & $\begin{array}{l}\text { Do not tank mix with fungicides. Wait a } \\
\text { minimum of } 48 \text { hours after application before } \\
\text { applying fungicides. }\end{array}$ \\
\hline \multirow[t]{3}{*}{ Bifenthrin } & $\begin{array}{l}\text { Attain TR } \\
\text { Attain TR Micro } \\
\text { Talstar Flowable } \\
\text { Talstar GF Prescription } \\
\end{array}$ & $G$ & \\
\hline & $\begin{array}{l}\text { Talstar N } \\
\text { Talstar Nursery Flowable }\end{array}$ & $F, G$ & $\begin{array}{l}\text { Nursery \& shade house }(\mathrm{F}) \text {; do not apply } \\
\text { more than } 0.2 \text { lbs. a.i. per acre per year for } \\
\text { outdoor applications. }\end{array}$ \\
\hline & Talstar Nursery Granular & $\mathrm{F}$ & $\begin{array}{l}\text { Larvae; for balled and containerized nursery } \\
\text { stocks. }\end{array}$ \\
\hline Chlorfenapyr & Pylon & $\mathrm{G}$ & $\begin{array}{l}\text { Early stage larvae (Bradysia sp.); non-edible } \\
\text { ornamentals; do not apply Pylon more than } \\
\text { two times consecutively or a total of three } \\
\text { times during a growing cycle (not more than } \\
41 \mathrm{fl} \text {. oz. of product/100 gal. / crop / season or } \\
0.64 \mathrm{lbs} \text {. of a.i. / } 100 \text { gal. / crop / season) } \\
\text { (start to finish for one ornamental crop). Do } \\
\text { not apply Pylon to consecutive crops in a } \\
\text { greenhouse unless the Pylon is applied in } \\
\text { combination with other effective miticides or } \\
\text { insecticides with a different mode of action. } \\
\text { Phytotoxicity is likely to occur to some } \\
\text { varieties of carnations, dianthus, kalanchoe, } \\
\text { poinsettia, roses, salvia, and zinnia. Additions } \\
\text { of crop oils, surfactants, and fertilizers or } \\
\text { other tank additives have been shown to } \\
\text { increase the likelihood of foliar injury. }\end{array}$ \\
\hline Chlorpyrifos & Duragard ME & $F, G$ & $\begin{array}{l}\text { Nursery (F); bedding plants, potted and cut } \\
\text { flowers. Direct spray to some open blooms } \\
\text { may cause petal drop. Do not spray on } \\
\text { kalanchoes. }\end{array}$ \\
\hline $\begin{array}{l}\text { Chlorpyrifos \& } \\
\text { Cyfluthrin }\end{array}$ & Duraplex TR & $G$ & \\
\hline Cyfluthrin & Decathlon $20 \mathrm{WP}$ & $F, G$ & \\
\hline Cyromazine & Citation & $F, G$ & $\begin{array}{l}\text { Container grown (F) or shade house grown } \\
\text { (F); will not control adult stages. }\end{array}$ \\
\hline Deltamethrin & Deltagard GC 5 SC & $\mathrm{F}$ & Nursery \\
\hline Diazinon & KnoxOut NL & $\mathrm{F}$ & Larvae; nursery \\
\hline
\end{tabular}


Table 6. Fungus Gnat (Diptera: Sciaridae) control measures available to commercial producers of any flower crop in Florida.

\begin{tabular}{|c|c|c|c|}
\hline Common Name & Trade Name/Formulation & $\begin{array}{l}\text { Production } \\
\text { Site }^{1}\end{array}$ & Notes from Label $^{2}$ \\
\hline Diflubenzuron & Adept & $F, G$ & $\begin{array}{l}\text { Shade house (F); do not make more than four } \\
\text { applications per crop. Do not apply to } \\
\text { poinsettias, hibiscus, and Rieger begonia. } \\
\text { Do not reuse potting media, which has been } \\
\text { treated with Adept. Do not apply to plants } \\
\text { grown on capillary water mats. }\end{array}$ \\
\hline Fenoxycarb & Precision & $F, G$ & $\begin{array}{l}\text { Bradysia spp.; Container grown (F) or shade } \\
\text { house grown }(F) \text {; will not control the adult } \\
\text { stages of insect pests. Do not exceed a } \\
\text { maximum of } 30 \text { applications per year for } \\
\text { greenhouse use. Applications should not be } \\
\text { made to poinsettia after bract formation. }\end{array}$ \\
\hline Permethrin & Astro & $\mathrm{G}$ & \\
\hline \multirow{2}{*}{$\begin{array}{l}\text { Pyrethrins \& Piperonyl } \\
\text { Butoxide }\end{array}$} & 1100 Pyrethrum TR & $G$ & \\
\hline & Pyrenone Crop Spray & $F, G$ & \\
\hline Pyriproxyfen & Distance IGR & $F, G$ & $\begin{array}{l}\text { For foliar spray application, apply Distance no } \\
\text { more than two times per cropping cycle or no } \\
\text { more than two times per } 6 \text { months. For } \\
\text { sprench application, if a second application is } \\
\text { needed, allow a minimum of } 21 \text { days between } \\
\text { applications. For drench application: Do not } \\
\text { drench plants more than one time per crop } \\
\text { cycle. Phytotoxicity has been observed on } \\
\text { salvia (Salvia spp.), ghost plant } \\
\text { (Graptopetalum paraguayense), schefflera } \\
\text { (Schefflera spp.), gardenia (Gardenia spp.), } \\
\text { and coral bells (Heuchera sanguinea). It is } \\
\text { therefore recommended that Distance not be } \\
\text { used on these plants. Do not apply to } \\
\text { poinsettia after bract formation. }\end{array}$ \\
\hline S-Kinoprene & Enstar II & $G$ & $\begin{array}{l}\text { Application should be made to poinsettia } \\
\text { before bract formation. Foliar damage on } \\
\text { some sensitive varieties can result. Some } \\
\text { varieties of roses, such as Yellow Blooded } \\
\text { roses, show delayed damage. Slight to } \\
\text { moderate injury has occurred on some } \\
\text { blooms under certain conditions, suggest } \\
\text { application be made in pre-bloom stage. }\end{array}$ \\
\hline $\begin{array}{l}\text { Steinernema } \\
\text { carpocapsae }\end{array}$ & Millenium & $F, G$ & Nursery $(F)$ \\
\hline Steinernema feltiae & Nemasys & $F, G$ & $\begin{array}{l}\text { Nursery }(\mathrm{F}) \text {; if fungus gnats are established it } \\
\text { may take } 2-3 \text { weeks before the number of } \\
\text { adults is noticeably reduced. Do not use } \\
\text { through drip irrigation or mist system. }\end{array}$ \\
\hline Thiamethoxam & Flagship 25WG & $F, G$ & $\begin{array}{l}\text { Shade house, container \& nursery grown }(F) \text {; } \\
\text { do not exceed } 8 \text { oz. per acre per crop or year, } \\
\text { which ever is shorter. }\end{array}$ \\
\hline
\end{tabular}


Table 6. Fungus Gnat (Diptera: Sciaridae) control measures available to commercial producers of any flower crop in Florida.

\begin{tabular}{|c|c|c|c|}
\hline Common Name & Trade Name/Formulation & $\begin{array}{l}\text { Production } \\
\text { Site }^{1}\end{array}$ & Notes from Label $^{2}$ \\
\hline \multicolumn{4}{|c|}{$\begin{array}{l}\text { Footnotes: } \\
1 \text { "F" indicates field production. "G" indicates greenhouse production. } \\
{ }^{2} \text { Notes are taken from product labels and restrict use to the condition indicated (suppression, beet armyworm, exposed } \\
\text { thrips, etc.), limit numbers or patterns of applications, provide phytotoxicity precautions, etc. }\end{array}$} \\
\hline
\end{tabular}

Table 7. Heliothis (tobacco budworm, etc.), Helicoverpa (corn earworm, etc.) control measures available to commercial producers of any flower crop in Florida. See also caterpillars (Lepidoptera).

\begin{tabular}{|c|c|c|c|}
\hline Common Name & Trade Name/Formulation & $\begin{array}{l}\text { Production } \\
\text { Site }^{1}\end{array}$ & Notes from Label $^{2}$ \\
\hline \multirow[t]{2}{*}{ Azadirachtin } & Azatin XL & $F, G$ & Corn earworm, tobacco budworm \\
\hline & Ornazin 3\% EC & $\mathrm{F}$ & Bollworm, corn earworm \\
\hline $\begin{array}{l}\text { Bacillus thuringiensis } \\
\text { aizawai }\end{array}$ & Xentari DF & $F, G$ & Heliothis, tobacco budworm \\
\hline $\begin{array}{l}\text { Bacillus thuringiensis } \\
\text { kurstaki }\end{array}$ & Javelin WG & $F, G$ & $\begin{array}{l}\text { Bollworm, Heliothis, Helicoverpa, tobacco } \\
\text { budworm }\end{array}$ \\
\hline $\begin{array}{l}\text { Bacillus thuringiensis } \\
\text { kurstaki strain ABTS-351 }\end{array}$ & Biobit HP & $F, G$ & Heliothis, tobacco budworm \\
\hline $\begin{array}{l}\text { Bacillus thuringiensis } \\
\text { kurstaki EG7841 }\end{array}$ & Crymax Bioinsecticide & $F, G$ & Tobacco budworm \\
\hline \multirow[t]{2}{*}{ Permethrin } & $\begin{array}{l}\text { Ambush } \\
\text { Ambush } 25 \text { W } \\
\text { Clean Crop Permethrin } 3.2 \\
\text { EC } \\
\text { Pounce } 3.2 \text { EC }\end{array}$ & $\mathrm{F}$ & $\begin{array}{l}\text { Heliothis; field grown nursery stock; do not } \\
\text { apply more than } 2.0 \text { lbs. a.i. per acre per } \\
\text { season. Marginal leaf burn may occur on: } \\
\text { Salvia, dieffenbachia. Application to blooming } \\
\text { plants may cause browning of petals. }\end{array}$ \\
\hline & $\begin{array}{l}\text { Pounce } 25 \text { WP } \\
\text { Pounce WSB }\end{array}$ & $\mathrm{F}$ & Heliothis; field grown nursery stock \\
\hline \multirow{2}{*}{$\begin{array}{l}\text { Pyrethrins \& Piperonyl } \\
\text { Butoxide }\end{array}$} & Pyrenone Crop Spray & $\mathrm{F}, \mathrm{G}$ & Bollworm, Heliothis, corn earworm \\
\hline & Pyreth-It & $F, G$ & Heliothis sp., corn earworm \\
\hline
\end{tabular}


Pesticide Options for Important Insect, Mite and Mollusk Pests of Commercial Flowers in....

Table 7. Heliothis (tobacco budworm, etc.), Helicoverpa (corn earworm, etc.) control measures available to commercial producers of any flower crop in Florida. See also caterpillars (Lepidoptera).

\begin{tabular}{||l|c|c|c||}
\hline \hline \multicolumn{1}{|c|}{ Common Name } & Trade Name/Formulation & $\begin{array}{c}\text { Production } \\
\text { Site }^{1}\end{array}$ & Notes from Label $^{2}$ \\
\hline $\begin{array}{l}\text { Footnotes: } \\
1 \text { "F" indicates field production. "G" indicates greenhouse production. } \\
{ }^{2} \text { Notes are taken from product labels and restrict use to the condition indicated (suppression, beet armyworm, exposed } \\
\text { thrips, etc.), limit numbers or patterns of applications, provide phytotoxicity precautions, etc. }\end{array}$ \\
\hline \hline
\end{tabular}

Table 8. Leafhopper (Homoptera: Cicadellidae) control measures available to commercial producers of any flower crop in Florida.

\begin{tabular}{|c|c|c|c|}
\hline Common Name & Trade Name/Formulation & $\begin{array}{l}\text { Production } \\
\text { Site }^{1}\end{array}$ & Notes from Label ${ }^{2}$ \\
\hline Acetamiprid & TriStar 70 WSP & $F, G$ & $\begin{array}{l}\text { Do not make more than five applications per } \\
\text { year. Do not reapply more than once every } 7 \\
\text { days. Do not apply more than } 22 \text { water } \\
\text { soluble packs per acre ( } 0.55 \mathrm{lb} \text {. a.i./acre) per } \\
\text { year. }\end{array}$ \\
\hline \multirow[t]{2}{*}{ Azadirachtin } & Azatin XL & $F, G$ & \\
\hline & Ornazin $3 \%$ EC & $\mathrm{F}$ & \\
\hline Azinphos-methyl & Guthion Solupak & $\mathrm{F}$ & Nursery stock \\
\hline $\begin{array}{l}\text { Beauveria bassiana } \\
\text { Strain GHA }\end{array}$ & Mycotrol 0 & $F$ & Edible flowers only \\
\hline \multirow[t]{2}{*}{ Bifenthrin } & $\begin{array}{l}\text { Talstar Flowable } \\
\text { Talstar GH Prescription } \\
\end{array}$ & $\mathrm{G}$ & \\
\hline & $\begin{array}{l}\text { Talstar N } \\
\text { Talstar Nursery Flowable }\end{array}$ & $F, G$ & $\begin{array}{l}\text { Nursery \& shade house }(\mathrm{F}) \text {; do not apply } \\
\text { more than } 0.2 \text { lbs. a.i. per acre per year for } \\
\text { outdoor applications. }\end{array}$ \\
\hline Buprofezin & $\begin{array}{l}\text { Talus Insect Growth } \\
\text { Regulator }\end{array}$ & $F, G$ & $\begin{array}{l}\text { Glassy-winged sharpshooter, variegated } \\
\text { leafhopper and brown planthopper; to } \\
\text { preserve usefulness of Talus, do not make } \\
\text { more than two consecutive applications. Prior } \\
\text { to subsequent applications, use an } \\
\text { alternative chemistry with a different mode of } \\
\text { action. }\end{array}$ \\
\hline \multirow[t]{2}{*}{ Carbaryl } & $\begin{array}{l}\text { Carbaryl } 4 \mathrm{~L} \\
\text { Carbaryl 80S } \\
\end{array}$ & $\mathrm{F}$ & \\
\hline & $\begin{array}{l}\text { Sevin } 80 \text { WSP } \\
\text { Sevin SL }\end{array}$ & $\mathrm{F}$ & $\begin{array}{l}\text { Application to wet foliage or during periods of } \\
\text { high humidity may cause injury to tender } \\
\text { foliage. }\end{array}$ \\
\hline
\end{tabular}


Table 8. Leafhopper (Homoptera: Cicadellidae) control measures available to commercial producers of any flower crop in Florida.

\begin{tabular}{|c|c|c|c|}
\hline Common Name & Trade Name/Formulation & $\begin{array}{l}\text { Production } \\
\text { Site }^{1}\end{array}$ & Notes from Label ${ }^{2}$ \\
\hline \multirow[t]{4}{*}{ Chlorpyrifos } & Chlorpyrifos Pro 2 & $\mathrm{~F}$ & Nursery stock in nurseries \\
\hline & Chlorpyrifos Pro 4 & $F, G$ & $\begin{array}{l}\text { Nursery }(\mathrm{F}) \text {; soil treatment, dormant spray of } \\
\text { tree pests, and pre-plant incorporation of field } \\
\text { grown nursery stock }(\mathrm{G}) \text {; some varieties of } \\
\text { azaleas, camellias, poinsettias, rose bushes, } \\
\text { or variegated ivy show varying degrees of } \\
\text { phytotoxicity. }\end{array}$ \\
\hline & Duragard ME & $F, G$ & $\begin{array}{l}\text { Nursery (F); bedding plants, potted and cut } \\
\text { flowers. Direct spray to some open blooms } \\
\text { may cause petal drop. Do not spray on } \\
\text { kalanchoes. }\end{array}$ \\
\hline & Dursban 50W & $\mathrm{F}$ & Nursery \\
\hline $\begin{array}{l}\text { Clarified Hydrophobic } \\
\text { Extract of Neem Oil }\end{array}$ & Triact 70 & $F, G$ & $\begin{array}{l}\text { Test before applying to carnation and the } \\
\text { flowers of: Impatiens, fuchsia, hibiscus, and } \\
\text { some roses. Most effective when applied } \\
\text { early to mid-morning or late afternoon. }\end{array}$ \\
\hline Cyfluthrin & Decathlon 20 WP & $\mathrm{F}, \mathrm{G}$ & \\
\hline Deltamethrin & Deltagard GC 5 SC & $\mathrm{F}$ & Nursery \\
\hline Diazinon & $\begin{array}{l}\text { Diazinon 50W } \\
\text { Diazinon AG } 500\end{array}$ & $\mathrm{~F}$ & $\begin{array}{l}\text { Precaution: Do not use on ferns, poinsettias, } \\
\text { hibiscus, pilea, and gardenia since injury to } \\
\text { the plants may occur. }\end{array}$ \\
\hline Fenpropathrin & Tame 2.4EC & $F, G$ & $\begin{array}{l}\text { Nursery \& shade house }(F) \text {; do not use less } \\
\text { than a } 7 \text { day re-treatment interval. }\end{array}$ \\
\hline Imidacloprid & Marathon II & $F, G$ & $\begin{array}{l}\text { For outdoor ornamentals do not apply more } \\
\text { than } 1.6 \text { pt. ( } 0.4 \text { lbs. a.i.) per acre per year. } \\
\text { Do not apply thru any type of irrigation } \\
\text { system. }\end{array}$ \\
\hline Malathion & $\begin{array}{l}\text { Atrapa } 5 \mathrm{E} \\
\text { Gowan Malathion } 8 \mathrm{~F} \\
\text { Malathion } 5 \mathrm{EC}\end{array}$ & $\mathrm{F}$ & Potato leafhopper, rose leafhopper \\
\hline \multirow[t]{5}{*}{ Permethrin } & Ambush 25W & $\mathrm{F}$ & $\begin{array}{l}\text { Field grown nursery stock; do not apply more } \\
\text { than } 2.0 \text { lbs. a.i. per acre per season. } \\
\text { Marginal leaf burn may occur on: Salvia, } \\
\text { dieffenbachia. Application to blooming plants } \\
\text { may cause browning of petals. }\end{array}$ \\
\hline & $\begin{array}{l}\text { Ambush } \\
\text { Pounce } 25 \text { WP } \\
\text { Pounce 3.2 EC } \\
\text { Pounce WSB } \\
\end{array}$ & $\mathrm{F}$ & Field grown nursery stock. \\
\hline & Astro & G & $\begin{array}{l}\text { Do not apply more than } 2.0 \mathrm{lb} \text {. a.i. per acre } \\
\text { per year. }\end{array}$ \\
\hline & $\begin{array}{l}\text { Clean Crop Permethrin } 3.2 \\
\text { EC }\end{array}$ & $\mathrm{F}$ & $\begin{array}{l}\text { Field grown nursery stock; marginal leaf burn } \\
\text { may occur on: Salvia, dieffenbachia. } \\
\text { Application to blooming plants may cause } \\
\text { browning of petals. Avoid spraying } \\
\text { chrysanthemum blooms. }\end{array}$ \\
\hline & Perm-Up 3.2EC & $\mathrm{F}$ & $\begin{array}{l}\text { Field grown nursery stock; marginal leaf burn } \\
\text { may occur on: Salvia, dieffenbachia. }\end{array}$ \\
\hline
\end{tabular}


Table 8. Leafhopper (Homoptera: Cicadellidae) control measures available to commercial producers of any flower crop in Florida.

\begin{tabular}{|c|c|c|c|}
\hline Common Name & Trade Name/Formulation & $\begin{array}{l}\text { Production } \\
\text { Site }^{1}\end{array}$ & Notes from Label $^{2}$ \\
\hline \multirow[t]{2}{*}{$\begin{array}{l}\text { Potassium Salts of Fatty } \\
\text { Acids }\end{array}$} & Insecticidal Soap 49.52 CF & $F, G$ & $\begin{array}{l}\text { Caution: Do not use on new transplants, } \\
\text { newly rooted cuttings, plants stressed by } \\
\text { drought, or when tender new foliage is } \\
\text { present. Avoid application when leaf } \\
\text { temperature exceeds } 85^{\circ} \mathrm{F} \text {. Note: Do not } \\
\text { use on crown of thorns and test on other } \\
\text { euphorbias for phytotoxicity. Do not use on } \\
\text { bleeding heart, lantana, lilies or sweetpeas. } \\
\text { Some varieties of azaleas, begonias, } \\
\text { camellias, fuschias, gardenias, orchids, and } \\
\text { impatiens have shown sensitivity. Do not } \\
\text { apply to poinsettias after bract coloration } \\
\text { begins. Test on chrysanthemums for varietal } \\
\text { sensitivity and do not apply to open blooms. } \\
\text { Do not apply to ornamentals stressed by } \\
\text { drought. Test on eugenia and euonymus for } \\
\text { varietal and drought sensitivity. }\end{array}$ \\
\hline & M-Pede & $F, G$ & $\begin{array}{l}\text { Tank mixes with chlorothalonil should be } \\
\text { avoided. Potential for injury increases on } \\
\text { plants stressed by heat, humidity, drought, or } \\
\text { insect, mite or disease pressure. Do not apply } \\
\text { to very sensitive plants such as: Cherimoya, } \\
\text { bleeding heart, or sweetpeas. The following } \\
\text { may be sensitive: Crown of thorns and other } \\
\text { euphorbia, fuchsia, gardenia, impatiens, } \\
\text { Asiatic and oriental lily varieties, lantana, } \\
\text { ornamental ivy, poinsettia, redbud, } \\
\text { schefflera, zebra plant and some succulents. } \\
\text { Flowers of the following may be injured when } \\
\text { sprayed: African violet, ageratum, azalea, } \\
\text { begonia, camellia, chrysanthemum, dahlia, } \\
\text { geranium, gloxinia, impatiens, lily, marigold, } \\
\text { orchid, pansy, petunia, poinsettia bracts, } \\
\text { rose, salvia, snapdragon, vinca, and zinnia. }\end{array}$ \\
\hline \multirow{2}{*}{$\begin{array}{l}\text { Pyrethrins \& Piperonyl } \\
\text { Butoxide }\end{array}$} & 1100 Pyrethrum TR & $\mathrm{G}$ & \\
\hline & $\begin{array}{l}\text { Pyrenone Crop Spray } \\
\text { Pyreth-It }\end{array}$ & $F, G$ & \\
\hline $\begin{array}{l}\text { Pyrethrins, Piperonyl } \\
\text { Butoxide \& Silicon } \\
\text { Dioxide }\end{array}$ & $\begin{array}{l}\text { Diatect Multipurpose } \\
\text { Insecticide II }\end{array}$ & $\mathrm{F}$ & \\
\hline $\begin{array}{l}\text { Pyrethrins, Rotenone \& } \\
\text { other Cube Resins }\end{array}$ & Pyrellin E.C. & $F, G$ & Apply in intervals of 7 days or less. \\
\hline
\end{tabular}


Table 8. Leafhopper (Homoptera: Cicadellidae) control measures available to commercial producers of any flower crop in Florida.

\begin{tabular}{|c|c|c|c|}
\hline Common Name & Trade Name/Formulation & $\begin{array}{l}\text { Production } \\
\text { Site }^{1}\end{array}$ & Notes from Label ${ }^{2}$ \\
\hline $\begin{array}{l}\text { Refined Petroleum } \\
\text { Distillate }\end{array}$ & Saf-T-Side Spray Oil & $\mathrm{F}$ & $\begin{array}{l}\text { This insecticide's mode of action is through } \\
\text { suffocation of eggs, larvae and nymphs of } \\
\text { insects and adults of soft bodied insects. This } \\
\text { mode of action necessitates total spray } \\
\text { coverage. Bedding plants; do not use in } \\
\text { combination with or immediately before or } \\
\text { after spraying with fungicides such as captan, } \\
\text { oxythioquinox or any product containing } \\
\text { sulfur. Also do not use with carbaryl (Sevin) } \\
\text { or dimethoate. Do not use with any product } \\
\text { whose label recommends the use of no oils. } \\
\text { Do not use in combination with NPK foliar } \\
\text { fertilizer applications. }\end{array}$ \\
\hline Tau-Fluvalinate & Mavrik Aquaflow & $F, G$ & $\begin{array}{l}\text { Repeat applications not more than four times } \\
\text { per month at 5-7 day intervals unless } \\
\text { otherwise noted in use directions. }\end{array}$ \\
\hline Thiamethoxam & Flagship 25WG & $\mathrm{F}, \mathrm{G}$ & $\begin{array}{l}\text { Shade house, container \& nursery grown }(F) \text {; } \\
\text { do not exceed } 8 \text { oz. per acre per crop or year, } \\
\text { which ever is shorter. }\end{array}$ \\
\hline \multicolumn{4}{|c|}{$\begin{array}{l}\text { Footnotes: } \\
1 \text { "F" indicates field production. "G" indicates greenhouse production. } \\
{ }^{2} \text { Notes are taken from product labels and restrict use to the condition indicated (suppression, beet armyworm, exposed } \\
\text { thrips, etc.), limit numbers or patterns of applications, provide phytotoxicity precautions, etc. }\end{array}$} \\
\hline
\end{tabular}

Table 9. Leafminer, dipterous (Diptera: Agromyzidae) control measures available to commercial producers of any flower crop in Florida.

\begin{tabular}{|c|c|c|c|}
\hline Common Name & Trade Name/Formulation & $\begin{array}{l}\text { Production } \\
\text { Site }^{1}\end{array}$ & Notes from Label ${ }^{2}$ \\
\hline Abamectin & Avid $0.15 \mathrm{EC}$ & $F, G$ & $\begin{array}{l}\text { Liriomyza leafminers; Phytotoxicity has been } \\
\text { observed on Shasta daisy. }\end{array}$ \\
\hline Acephate $^{3}$ & 1300 Orthene TR & $\mathrm{G}$ & \\
\hline $\begin{array}{l}\text { Acephate }^{3} \& \\
\text { Fenpropathrin }^{3}\end{array}$ & Tame/Orthene TR & $\mathrm{G}$ & $\begin{array}{l}\text { Do not apply within } 48 \text { hours of a previous } \\
\text { application. }\end{array}$ \\
\hline Azadirachtin & $\begin{array}{l}\text { Azatin } \mathrm{XL} \\
\text { Ornazin 3\% EC }\end{array}$ & $F, G$ & \\
\hline \multirow[t]{2}{*}{ Bifenthrin $^{3}$} & $\begin{array}{l}\text { Talstar Flowable } \\
\text { Talstar GH Prescription }\end{array}$ & $G$ & \\
\hline & $\begin{array}{l}\text { Talstar N } \\
\text { Talstar Nursery Flowable }\end{array}$ & $F, G$ & $\begin{array}{l}\text { Nursery \& shade house (F); do not apply } \\
\text { more than } 0.2 \text { lbs. a.i. per acre per year for } \\
\text { outdoor applications. }\end{array}$ \\
\hline
\end{tabular}


Pesticide Options for Important Insect, Mite and Mollusk Pests of Commercial Flowers in....

Table 9. Leafminer, dipterous (Diptera: Agromyzidae) control measures available to commercial producers of any flower crop in Florida.

\begin{tabular}{|c|c|c|c|}
\hline Common Name & Trade Name/Formulation & $\begin{array}{l}\text { Production } \\
\text { Site }^{1}\end{array}$ & Notes from Label $^{2}$ \\
\hline \multirow[t]{4}{*}{ Chlorpyrifos $^{3}$} & Chlorpyrifos Pro 2 & $\mathrm{~F}$ & Nursery stock in nurseries \\
\hline & Chlorpyrifos Pro 4 & $F, G$ & $\begin{array}{l}\text { Nursery }(F) \text {; soil treatment, dormant spray of } \\
\text { tree pests, and pre-plant incorporation of field } \\
\text { grown nursery stock }(G) \text {; some varieties of } \\
\text { azaleas, camellias, poinsettias, rose bushes, } \\
\text { or variegated ivy show varying degrees of } \\
\text { phytotoxicity. }\end{array}$ \\
\hline & Duragard ME & $F, G$ & $\begin{array}{l}\text { Nursery }(\mathrm{F}) \text {; bedding plants, potted and cut } \\
\text { flowers. Direct spray to some open blooms } \\
\text { may cause petal drop. Do not spray on } \\
\text { kalanchoes. }\end{array}$ \\
\hline & Dursban 50W & $\mathrm{F}$ & Nursery \\
\hline Cyromazine & Citation & $F, G$ & $\begin{array}{l}\text { Container grown (F) or shade house grown } \\
(F) \text {; will not control adult stages. Leafminers } \\
\text { can develop resistance, therefore, do not use } \\
\text { as the only control for leafminers, make no } \\
\text { more than three applications at 7-day } \\
\text { intervals, then switch to Avid as an alternative } \\
\text { control for the same period, then Citation may } \\
\text { be applied again at 7-day intervals if } \\
\text { necessary. Do not make more than six } \\
\text { applications to one crop. }\end{array}$ \\
\hline Deltamethrin $^{3}$ & Deltagard GC 5 SC & $\mathrm{F}$ & Nursery \\
\hline \multirow[t]{2}{*}{ Diazinon $^{3}$} & $\begin{array}{l}\text { Diazinon 50W } \\
\text { Diazinon AG } 500\end{array}$ & $\bar{F}$ & $\begin{array}{l}\text { Precaution: Do not use on ferns, poinsettias, } \\
\text { hibiscus, pilea, and gardenia since injury to } \\
\text { the plants may occur. }\end{array}$ \\
\hline & KnoxOut NL & $\mathrm{F}$ & Nursery \\
\hline Fenpropathrin $^{3}$ & Tame 2.4EC & $F, G$ & $\begin{array}{l}\text { Nursery \& shade house }(F) \text {; do not use less } \\
\text { than a } 7 \text { day re-treatment interval. }\end{array}$ \\
\hline
\end{tabular}


Table 9. Leafminer, dipterous (Diptera: Agromyzidae) control measures available to commercial producers of any flower crop in Florida.

\begin{tabular}{|c|c|c|c|}
\hline Common Name & Trade Name/Formulation & $\begin{array}{l}\text { Production } \\
\text { Site }^{1}\end{array}$ & Notes from Label ${ }^{2}$ \\
\hline \multirow[t]{3}{*}{ Imidacloprid } & Marathon $1 \%$ Granular & $F, G$ & $\begin{array}{l}\text { Nursery }(\mathrm{F}) \text {; for outdoor ornamentals grown } \\
\text { in beds, applications can not exceed a total of } \\
40 \mathrm{lbs} \text {. per acre per year. On plants with a } \\
\text { production cycle of less than } 1 \text { year, } \\
\text { application is not to exceed a frequency of } \\
\text { more than once each } 16 \text { weeks for a } \\
\text { particular plant. On stock plants and woody } \\
\text { crops with a production cycle of greater than } \\
1 \text { year, application may not exceed once a } \\
\text { year. }\end{array}$ \\
\hline & Marathon $60 \mathrm{WP}$ & $F, G$ & $\begin{array}{l}\text { Nursery }(\mathrm{F}) \text {; this product is to be applied as a } \\
\text { soil treatment only. For outdoor ornamentals } \\
\text { grown in beds applications can not exceed a } \\
\text { total of } 10.7 \text { oz. ( } 0.4 \mathrm{lbs} \text {. Active ingredient) } \\
\text { per acre per year. On plants with a production } \\
\text { cycle of less than } 1 \text { year, application is not to } \\
\text { exceed a frequency of more than once each } \\
16 \text { weeks for a particular plant. On stock } \\
\text { plants and woody crops with a production } \\
\text { cycle of greater than } 1 \text { year, application may } \\
\text { not exceed once a year. }\end{array}$ \\
\hline & Marathon II & $F, G$ & $\begin{array}{l}\text { For outdoor ornamentals do not apply more } \\
\text { than } 1.6 \text { pt. }(0.4 \mathrm{lbs} \text {. a.i.) per acre per year. } \\
\text { Do not apply thru any type of irrigation } \\
\text { system. }\end{array}$ \\
\hline Lambda-cyhalothrin $^{3}$ & Scimitar GC & $F, G$ & $\begin{array}{l}\text { Nursery \& shade house }(\mathrm{F}) \text {; do not apply } \\
\text { more than } 0.36 \text { lbs. a.i. ( } 52.4 \text { fl. oz. of } \\
\text { concentrate) per acre per year. }\end{array}$ \\
\hline Novaluron & Pedestal & $F, G$ & $\begin{array}{l}\text { Container grown plants; nursery \& shade } \\
\text { house (F); do not apply more than once every } \\
30 \text { days. Do not make more than two } \\
\text { applications per crop year. Do not apply more } \\
\text { than } 52 \text { fl. oz. per acre per year per crop. Do } \\
\text { not apply to poinsettias. Resistance } \\
\text { management: Do not apply in successive } \\
\text { applications, use only one application before } \\
\text { rotation with other classes of insecticides with } \\
\text { different modes of action. Use at least two } \\
\text { alternative products between applications of } \\
\text { Pedestal. Do not use more than once within } \\
\text { each generation cycle. }\end{array}$ \\
\hline Oxydemeton-methy $\left.\right|^{3}$ & Metasystox-R & $\mathrm{F}$ & $\begin{array}{l}\text { Field grown nursery stock; do not make more } \\
\text { than } 2 \text { applications per season. }\end{array}$ \\
\hline
\end{tabular}


Table 9. Leafminer, dipterous (Diptera: Agromyzidae) control measures available to commercial producers of any flower crop in Florida.

\begin{tabular}{|c|c|c|c|}
\hline Common Name & Trade Name/Formulation & $\begin{array}{l}\text { Production } \\
\text { Site }^{1}\end{array}$ & Notes from Label ${ }^{2}$ \\
\hline \multirow[t]{5}{*}{ Permethrin $^{3}$} & Ambush 25W & $\mathrm{F}$ & $\begin{array}{l}\text { Field grown nursery stock; do not apply more } \\
\text { than } 2.0 \text { lbs. a.i. per acre per season. } \\
\text { Marginal leaf burn may occur on: Salvia, } \\
\text { dieffenbachia. Application to blooming plants } \\
\text { may cause browning of petals. }\end{array}$ \\
\hline & $\begin{array}{l}\text { Ambush } \\
\text { Pounce } 25 \text { WP } \\
\text { Pounce } 3.2 \text { EC } \\
\text { Pounce WSB } \\
\end{array}$ & $\mathrm{F}$ & Field grown nursery stock \\
\hline & Astro & $\bar{G}$ & $\begin{array}{l}\text { Do not apply more than } 2.0 \mathrm{lb} \text {. a.i. per acre } \\
\text { per year. }\end{array}$ \\
\hline & $\begin{array}{l}\text { Clean Crop Permethrin } 3.2 \\
\text { EC }\end{array}$ & $\mathrm{F}$ & $\begin{array}{l}\text { Field grown nursery stock; marginal leaf burn } \\
\text { may occur on: Salvia, dieffenbachia. } \\
\text { Application to blooming plants may cause } \\
\text { browning of petals. Avoid spraying } \\
\text { chrysanthemum blooms. }\end{array}$ \\
\hline & Perm-Up 3.2EC & $\mathrm{F}$ & $\begin{array}{l}\text { Field grown nursery stock; marginal leaf burn } \\
\text { may occur on: Salvia, dieffenbachia. }\end{array}$ \\
\hline $\begin{array}{l}\text { Potssium Salts of Fatty } \\
\text { Acids }\end{array}$ & M-Pede & F. G & $\begin{array}{l}\text { For control of leafminers must combine with } \\
\text { other pesticides. Tank mixes with } \\
\text { chlorothalonil should be avoided. Potential for } \\
\text { injury increases on plants stressed by heat, } \\
\text { humidity, drought, or insect, mite or disease } \\
\text { pressure. Do not apply to very sensitive } \\
\text { plants such as: Cherimoya, bleeding heart, or } \\
\text { sweetpeas. The following may be sensitive: } \\
\text { Crown of thorns and other euphorbia, fuchsia, } \\
\text { gardenia, impatiens, Asiatic and oriental lily } \\
\text { varieties, lantana, ornamental ivy, poinsettia, } \\
\text { redbud, schefflera, zebra plant and some } \\
\text { succulents. Flowers of the following may be } \\
\text { injured when sprayed: African violet, } \\
\text { ageratum, azalea, begonia, camellia, } \\
\text { chrysanthemum, dahlia, geranium, gloxinia, } \\
\text { impatiens, lily, marigold, orchid, pansy, } \\
\text { petunia, poinsettia bracts, rose, salvia, } \\
\text { snapdragon, vinca, and zinnia. }\end{array}$ \\
\hline $\begin{array}{l}\text { Pyrethrins \& Piperonyl } \\
\text { Butoxide }\end{array}$ & Pyrenone Crop Spray & $F, G$ & \\
\hline $\begin{array}{l}\text { Pyrethrins, Piperonyl } \\
\text { Butoxide \& Silicon } \\
\text { Dioxide }\end{array}$ & $\begin{array}{l}\text { Diatect Multipurpose } \\
\text { Insecticide II }\end{array}$ & $\mathrm{F}$ & \\
\hline $\begin{array}{l}\text { Pyrethrins, Rotenone \& } \\
\text { other Cube Resins }\end{array}$ & Pyrellin E.C. & $F, G$ & Apply in intervals of 7 days or less. \\
\hline
\end{tabular}


Table 9. Leafminer, dipterous (Diptera: Agromyzidae) control measures available to commercial producers of any flower crop in Florida.

\begin{tabular}{|c|c|c|c|}
\hline Common Name & Trade Name/Formulation & $\begin{array}{l}\text { Production } \\
\text { Site }^{1}\end{array}$ & Notes from Label ${ }^{2}$ \\
\hline \multirow[t]{2}{*}{$\begin{array}{l}\text { Refined Petroleum } \\
\text { Distillate }\end{array}$} & Saf-T-Side Spray Oil & $\mathrm{F}$ & $\begin{array}{l}\text { This insecticide's mode of action is through } \\
\text { suffocation of eggs, larvae and nymphs of } \\
\text { insects and adults of soft bodied insects. This } \\
\text { mode of action necessitates total spray } \\
\text { coverage. Bedding plants; do not use in } \\
\text { combination with or immediately before or } \\
\text { after spraying with fungicides such as captan, } \\
\text { oxythioquinox or any product containing } \\
\text { sulfur. Also do not use with carbaryl (Sevin) } \\
\text { or dimethoate. Do not use with any product } \\
\text { whose label recommends the use of no oils. } \\
\text { Do not use in combination with NPK foliar } \\
\text { fertilizer applications. }\end{array}$ \\
\hline & Ultra-Fine Oil & $\mathrm{F}$ & $\begin{array}{l}\text { Caution: Spray no more than } 4 \text { consecutive } \\
\text { sprays. The frequency of consecutive sprays } \\
\text { should not exceed once every } 2 \text { weeks. Early } \\
\text { morning applications are recommended. Do } \\
\text { not tank mix with insecticide or miticide whose } \\
\text { label indicates that it should not be used with } \\
\text { oil. Do not use with captan, chlorothalonil, } \\
\text { dimethoate, methiocarb, oxythioquinox, } \\
\text { propargite, or any product containing sulfur. } \\
\text { This list is not exhaustive; therefore for } \\
\text { products not included in this list consult label } \\
\text { for compatibility information. }\end{array}$ \\
\hline Spinosad & Conserve SC & $F, G$ & $\begin{array}{l}\text { When used in greenhouse or areas of } \\
\text { commercial production of herbaceous } \\
\text { (non-woody) ornamentals in nurseries } \\
\text { (including plant propagation beds): Do not } \\
\text { apply more than } 10 \text { times in a 12-month } \\
\text { period per crop regardless of the pest being } \\
\text { treated. Because generations of a specific } \\
\text { pest may overlap, rotate control products and } \\
\text { never apply more than three consecutive } \\
\text { applications of Conserve SC or products } \\
\text { containing the same a.i. or products with the } \\
\text { same mode of action. Except for } \\
\text { greenhouses and structures that can be } \\
\text { altered to be closed or open, do not reapply } \\
\text { within less than } 7 \text { days. }\end{array}$ \\
\hline \multicolumn{4}{|c|}{$\begin{array}{l}\text { Footnotes: } \\
1 \text { "F" indicates field production. "G" indicates greenhouse production. } \\
{ }^{2} \text { Notes are taken from product labels and restrict use to the condition indicated (suppression, beet armyworm, exposed } \\
\text { thrips, etc.), limit numbers or patterns of applications, provide phytotoxicity precautions, etc. } \\
{ }^{3} \text { Liriomyza trifolii leafminers have become resistant to organophosphate and pyrethroid insecticides. Infrequent use may } \\
\text { be effective, but repeated use may be ineffective, depending on the history of the target population. }\end{array}$} \\
\hline
\end{tabular}


Table 10. Leafrollers \& leaftiers (Lepidoptera: Torticidae) control measures available to commercial producers of any flower crop in Florida. Also see Caterpillars (Lepidoptera).

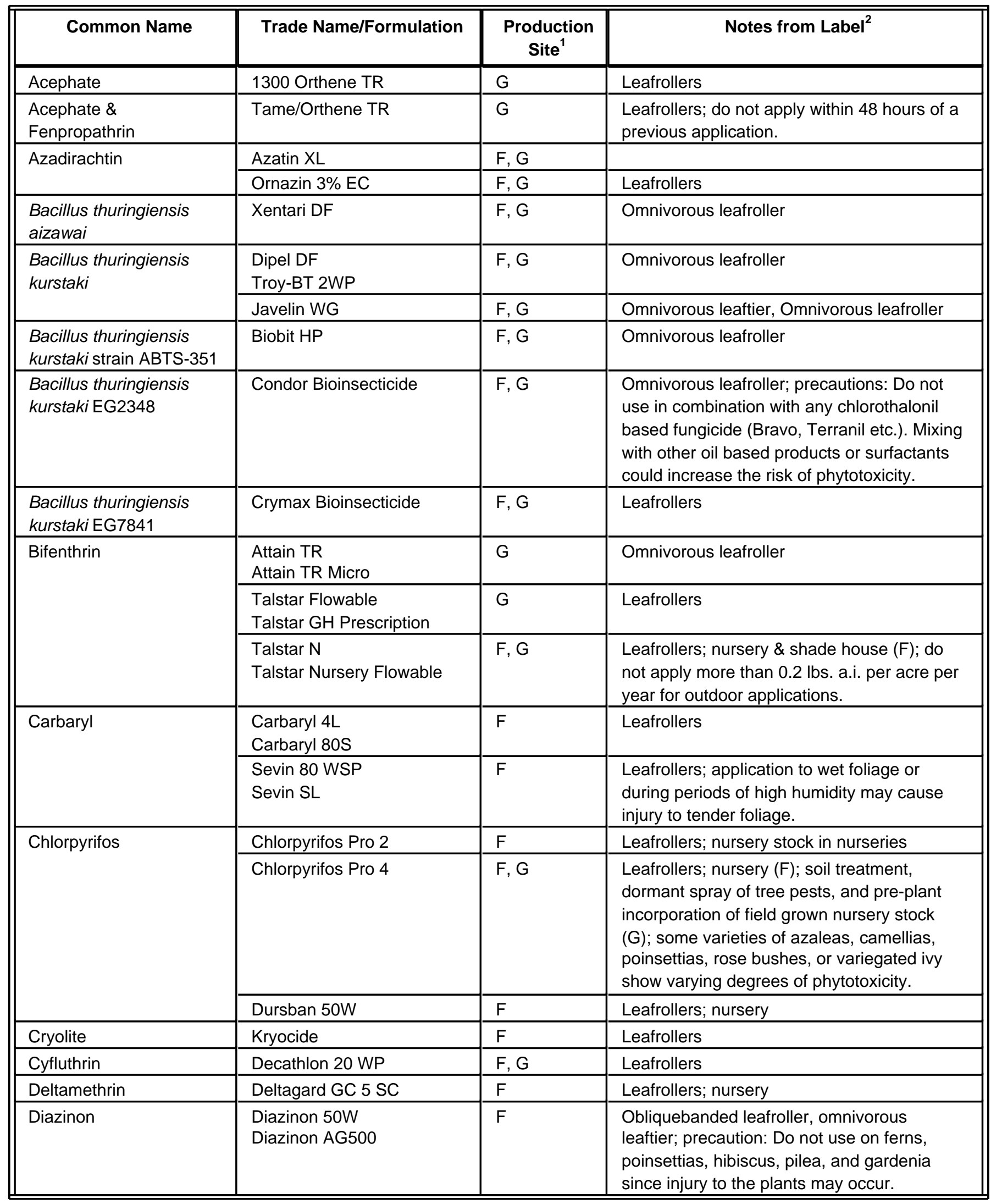


Table 10. Leafrollers \& leaftiers (Lepidoptera: Torticidae) control measures available to commercial producers of any flower crop in Florida. Also see Caterpillars (Lepidoptera).

\begin{tabular}{|c|c|c|c|}
\hline Common Name & Trade Name/Formulation & $\begin{array}{l}\text { Production } \\
\text { Site }^{1}\end{array}$ & Notes from Label ${ }^{2}$ \\
\hline Fenpropathrin & Tame 2.4EC & $F, G$ & $\begin{array}{l}\text { Leafrollers; nursery \& shade house }(\mathrm{F}) \text {; do } \\
\text { not use less than a } 7 \text { day re-treatment } \\
\text { interval. }\end{array}$ \\
\hline Lambda-cyhalothrin & Scimitar GC & $F, G$ & $\begin{array}{l}\text { Leafrollers; nursery \& shade house (F); do } \\
\text { not apply more than } 0.36 \text { lbs. a.i. ( } 52.4 \mathrm{fl} . \text { oz. } \\
\text { of concentrate) per acre per year. }\end{array}$ \\
\hline Naled & Dibrom 8 Emulsive & $\mathrm{G}$ & $\begin{array}{l}\text { Leafrollers; vapor treatment; spotting, } \\
\text { bronzing or localized burning can occur under } \\
\text { certain climatic conditions such as slow } \\
\text { drying and high humidity or stress caused by } \\
\text { drought or high temperature. White Butterfly } \\
\text { and Golden Rapture roses, Pink Champagne } \\
\text { chrysanthemums, green wandering jew, } \\
\text { poinsettias and Dutchman's pipe may be } \\
\text { injured by vapor. }\end{array}$ \\
\hline Permethrin & Astro & $\mathrm{G}$ & $\begin{array}{l}\text { Leafrollers; do not apply more than } 2.0 \mathrm{lb} \text {. a.i. } \\
\text { per acre per year. }\end{array}$ \\
\hline $\begin{array}{l}\text { Pyrethrins \& Piperonyl } \\
\text { Butoxide }\end{array}$ & $\begin{array}{l}\text { Pyrenone Crop Spray } \\
\text { Pyreth-It }\end{array}$ & $F, G$ & \\
\hline $\begin{array}{l}\text { Pyrethrins, Piperonyl } \\
\text { Butoxide \& Silicon } \\
\text { Dioxide }\end{array}$ & $\begin{array}{l}\text { Diatect Multipurpose } \\
\text { Insecticide II }\end{array}$ & $\mathrm{F}$ & Leafrollers \\
\hline $\begin{array}{l}\text { Pyrethrins, Rotenone \& } \\
\text { other Cube Resins }\end{array}$ & Pyrellin E.C. & $F, G$ & $\begin{array}{l}\text { Leafrollers; Apply in intervals of } 7 \text { days or } \\
\text { less. }\end{array}$ \\
\hline \multirow[t]{2}{*}{$\begin{array}{l}\text { Refined Petroleum } \\
\text { Distillate }\end{array}$} & Saf-T-Side Spray Oil & $\mathrm{F}$ & $\begin{array}{l}\text { This insecticide's mode of action is through } \\
\text { suffocation of eggs, larvae and nymphs of } \\
\text { insects and adults of soft bodied insects. This } \\
\text { mode of action necessitates total spray } \\
\text { coverage. Bedding plants; do not use in } \\
\text { combination with or immediately before or } \\
\text { after spraying with fungicides such as captan, } \\
\text { oxythioquinox or any product containing } \\
\text { sulfur. Also do not use with carbaryl (Sevin) } \\
\text { or dimethoate. Do not use with any product } \\
\text { whose label recommends the use of no oils. } \\
\text { Do not use in combination with NPK foliar } \\
\text { fertilizer applications. }\end{array}$ \\
\hline & Ultra-Fine Oil & $\mathrm{F}$ & $\begin{array}{l}\text { Leafrollers; caution: Spray no more than } 4 \\
\text { consecutive sprays. The frequency of } \\
\text { consecutive sprays should not exceed once } \\
\text { every } 2 \text { weeks. Early morning applications } \\
\text { are recommended. Do not tank mix with } \\
\text { insecticide or miticide whose label indicates } \\
\text { that it should not be used with oil. Do not use } \\
\text { with captan, chlorothalonil, dimethoate, } \\
\text { methiocarb, oxythioquinox, propargite, or any } \\
\text { product containing sulfur. This list is not } \\
\text { exhaustive; therefore for products not } \\
\text { included in this list consult label for } \\
\text { compatibility information. }\end{array}$ \\
\hline
\end{tabular}


Table 10. Leafrollers \& leaftiers (Lepidoptera: Torticidae) control measures available to commercial producers of any flower crop in Florida. Also see Caterpillars (Lepidoptera).

\begin{tabular}{|c|c|c|c|}
\hline Common Name & Trade Name/Formulation & $\begin{array}{l}\text { Production } \\
\text { Site }^{1}\end{array}$ & Notes from Label ${ }^{2}$ \\
\hline Spinosad & Conserve SC & $F, G$ & $\begin{array}{l}\text { Obliquebanded leafroller; when used in } \\
\text { greenhouse or areas of commercial } \\
\text { production of herbaceous (non-woody) } \\
\text { ornamentals in nurseries (including plant } \\
\text { propagation beds): Do not apply more than } \\
10 \text { times in a 12-month period per crop } \\
\text { regardless of the pest being treated. Because } \\
\text { generations of a specific pest may overlap, } \\
\text { rotate control products and never apply more } \\
\text { than three consecutive applications of } \\
\text { Conserve SC or products containing the } \\
\text { same a.i. or products with the same mode of } \\
\text { action. Except for greenhouses and } \\
\text { structures that can be altered to be closed or } \\
\text { open, do not reapply within less than } 7 \text { days. }\end{array}$ \\
\hline \multicolumn{4}{|c|}{$\begin{array}{l}\text { Footnotes: } \\
1 \text { "F" indicates field production. "G" indicates greenhouse production. } \\
{ }^{2} \text { Notes are taken from product labels and restrict use to the condition indicated (suppression, beet armyworm, exposed } \\
\text { thrips, etc.), limit numbers or patterns of applications, provide phytotoxicity precautions, etc. }\end{array}$} \\
\hline
\end{tabular}

Table 11. Looper (Lepidoptera: Noctuidae) control measures available to commercial producers of any flower crop in Florida. Also see Caterpillars (Lepidoptera).

\begin{tabular}{|c|c|c|c|}
\hline Common Name & Trade Name/Formulation & $\begin{array}{l}\text { Production } \\
\text { Site }^{1}\end{array}$ & Notes from Label $^{2}$ \\
\hline Acephate & 1300 Orthene TR & $G$ & Cabbage loopers \\
\hline $\begin{array}{l}\text { Acephate \& } \\
\text { Fenpropathrin }\end{array}$ & Tame/Orthene TR & $G$ & $\begin{array}{l}\text { Cabbage loopers; do not apply within } 48 \\
\text { hours of a previous application. }\end{array}$ \\
\hline Azadirachtin & $\begin{array}{l}\text { Azatin XL } \\
\text { Ornazin 3\% EC }\end{array}$ & $F, G$ & \\
\hline $\begin{array}{l}\text { Bacillus thuringiensis } \\
\text { aizawai }\end{array}$ & Xentari DF & $F, G$ & \\
\hline $\begin{array}{l}\text { Bacillus thuringiensis } \\
\text { kurstaki }\end{array}$ & $\begin{array}{l}\text { Dipel DF } \\
\text { Javelin WG } \\
\text { Troy-BT 2WP }\end{array}$ & $F, G$ & \\
\hline $\begin{array}{l}\text { Bacillus thuringiensis } \\
\text { kurstaki ABTS-351 }\end{array}$ & Biobit HP & $F, G$ & \\
\hline $\begin{array}{l}\text { Bacillus thuringiensis } \\
\text { kurstaki EG2348 }\end{array}$ & Condor Bioinsecticide & $F, G$ & $\begin{array}{l}\text { Precautions: Do not use in combination with } \\
\text { any chlorothalonil based fungicide (Bravo, } \\
\text { Terranil etc.). Mixing with other oil based } \\
\text { products or surfactants could increase the } \\
\text { risk of phytotoxicity. }\end{array}$ \\
\hline $\begin{array}{l}\text { Bacillus thuringiensis } \\
\text { kurstaki EG7841 }\end{array}$ & Crymax Bioinsecticide & $F, G$ & \\
\hline
\end{tabular}


Table 11. Looper (Lepidoptera: Noctuidae) control measures available to commercial producers of any flower crop in Florida. Also see Caterpillars (Lepidoptera).

\begin{tabular}{|c|c|c|c|}
\hline Common Name & Trade Name/Formulation & $\begin{array}{l}\text { Production } \\
\text { Site }^{1}\end{array}$ & Notes from Label ${ }^{2}$ \\
\hline \multirow[t]{5}{*}{ Permethrin } & Ambush 25W & $\mathrm{F}$ & $\begin{array}{l}\text { Cabbage looper; field grown nursery stock; } \\
\text { do not apply more than } 2.0 \mathrm{lbs} \text {. a.i. per acre } \\
\text { per season. Marginal leaf burn may occur on: } \\
\text { Salvia, dieffenbachia. Application to blooming } \\
\text { plants may cause browning of petals. }\end{array}$ \\
\hline & $\begin{array}{l}\text { Ambush } \\
\text { Pounce 25 WP } \\
\text { Pounce } 3.2 \text { EC } \\
\text { Pounce WSB }\end{array}$ & $\mathrm{F}$ & Cabbage looper; field grown nursery stock \\
\hline & Astro & $\mathrm{G}$ & $\begin{array}{l}\text { Cabbage looper; do not apply more than } 2.0 \\
\text { lb. a.i. per acre per year. }\end{array}$ \\
\hline & $\begin{array}{l}\text { Clean Crop Permethrin } 3.2 \\
\text { EC }\end{array}$ & $\mathrm{F}$ & $\begin{array}{l}\text { Cabbage looper; field grown nursery stock; } \\
\text { marginal leaf burn may occur on: Salvia, } \\
\text { dieffenbachia. Application to blooming plants } \\
\text { may cause browning of petals. Avoid } \\
\text { spraying chrysanthemum blooms. }\end{array}$ \\
\hline & Perm-Up 3.2EC & $\mathrm{F}$ & $\begin{array}{l}\text { Cabbage looper; field grown nursery stock; } \\
\text { marginal leaf burn may occur on: Salvia, } \\
\text { dieffenbachia. }\end{array}$ \\
\hline \multirow{2}{*}{$\begin{array}{l}\text { Pyrethrins \& Piperonyl } \\
\text { Butoxide }\end{array}$} & Pyrenone Crop Spray & $\mathrm{F}, \mathrm{G}$ & \\
\hline & Pyreth-It & $\mathrm{F}, \mathrm{G}$ & Cabbage looper \\
\hline $\begin{array}{l}\text { Pyrethrins, Piperonyl } \\
\text { Butoxide \& Silicon } \\
\text { Dioxide }\end{array}$ & $\begin{array}{l}\text { Diatect Multipurpose } \\
\text { Insecticide II }\end{array}$ & $\mathrm{F}$ & \\
\hline $\begin{array}{l}\text { Pyrethrins, Rotenone \& } \\
\text { other Cube Resins }\end{array}$ & Pyrellin E.C. & $F, G$ & Apply in intervals of 7 days or less. \\
\hline Tebufenozide & Confirm T/O & $\mathrm{F}, \mathrm{G}$ & $\begin{array}{l}\text { Hemlock looper; nursery }(\mathrm{F}) \text {; do not use to } \\
\text { control more than three consecutive } \\
\text { generations of pests in a cropping area (field) } \\
\text { regardless of the crop rotation on that field. If } \\
\text { you are unsure of the number of generations } \\
\text { treated do not use more than four times } \\
\text { within any } 80 \text {-day interval. If Confirm has } \\
\text { been applied four times in } 80 \text { days or less, } \\
\text { allow at least } 40 \text { days to pass before making } \\
\text { additional applications. }\end{array}$ \\
\hline \multicolumn{4}{|c|}{$\begin{array}{l}\text { Footnotes: } \\
1 \text { "F" indicates field production. "G" indicates greenhouse production. } \\
{ }^{2} \text { Notes are taken from product labels and restrict use to the condition indicated (suppression, beet armyworm, exposed } \\
\text { thrips, etc.), limit numbers or patterns of applications, provide phytotoxicity precautions, etc. }\end{array}$} \\
\hline
\end{tabular}


Table 12. Mealybug (Homoptera: Pseudococcidae) control measures available to commercial producers of any flower crop in Florida.

\begin{tabular}{|c|c|c|c|}
\hline Common Name & Trade Name/Formulation & $\begin{array}{l}\text { Production } \\
\text { Site }^{1}\end{array}$ & Notes from Label ${ }^{2}$ \\
\hline Acephate & 1300 Orthene TR & $\mathrm{G}$ & \\
\hline Acephate \& Fenpropathrin & Tame/Orthene TR & $G$ & $\begin{array}{l}\text { Do not apply within } 48 \text { hours of a previous } \\
\text { application. }\end{array}$ \\
\hline Acetamiprid & TriStar 70 WSP & $F, G$ & $\begin{array}{l}\text { Do not make more than five applications per } \\
\text { year. Do not reapply more than once every } 7 \\
\text { days. Do not apply more than } 22 \text { water } \\
\text { soluble packs per acre ( } 0.55 \mathrm{lb} \text {. a.i./acre) per } \\
\text { year. }\end{array}$ \\
\hline Azadirachtin & $\begin{array}{l}\text { Azatin } X L \\
\text { Ornazin } 3 \% E C\end{array}$ & $F, G$ & \\
\hline $\begin{array}{l}\text { Beauveria bassiana } \\
\text { ATCC } 74040\end{array}$ & Naturalis $L$ & $F, G$ & $\begin{array}{l}\text { Do not tank mix with fungicides. Wait a } \\
\text { minimum of } 48 \text { hours after application before } \\
\text { applying fungicides. }\end{array}$ \\
\hline \multirow{3}{*}{$\begin{array}{l}\text { Beauveria bassiana } \\
\text { Strain GHA }\end{array}$} & Botanigard $22 \mathrm{WP}$ & $\mathrm{F}, \mathrm{G}$ & Nursery \& shade house $(F)$ \\
\hline & Botanigard ES & $\mathrm{F}, \mathrm{G}$ & Nursery $(\mathrm{F})$ \\
\hline & Mycotrol O & $\mathrm{F}, \mathrm{G}$ & Edible flowers only \\
\hline \multirow[t]{4}{*}{ Bifenthrin } & $\begin{array}{l}\text { Attain TR } \\
\text { Attain TR Micro }\end{array}$ & $\mathrm{G}$ & \\
\hline & $\begin{array}{l}\text { Talstar Flowable } \\
\text { Talstar GH Prescription }\end{array}$ & $\mathrm{G}$ & \\
\hline & $\begin{array}{l}\text { Talstar N } \\
\text { Talstar Nursery Flowable }\end{array}$ & $F, G$ & $\begin{array}{l}\text { Nursery \& shade house }(\mathrm{F}) \text {; do not apply } \\
\text { more than } 0.2 \text { lbs. a.i. per acre per year for } \\
\text { outdoor applications. }\end{array}$ \\
\hline & Talstar Nursery Granular & $\mathrm{F}$ & For balled and containerized nursery stocks. \\
\hline Buprofezin & $\begin{array}{l}\text { Talus Insect Growth } \\
\text { Regulator }\end{array}$ & $F, G$ & $\begin{array}{l}\text { Citrus, longtailed and Mexican mealybug; to } \\
\text { preserve usefulness of Talus, do not make } \\
\text { more than two consecutive applications. Prior } \\
\text { to subsequent applications, use an } \\
\text { alternative chemistry with a different mode of } \\
\text { action. }\end{array}$ \\
\hline \multirow[t]{2}{*}{ Carbaryl } & $\begin{array}{l}\text { Carbaryl } 4 \mathrm{~L} \\
\text { Carbaryl 80S } \\
\end{array}$ & $\mathrm{F}$ & \\
\hline & $\begin{array}{l}\text { Sevin } 80 \text { WSP } \\
\text { Sevin SL }\end{array}$ & $\mathrm{F}$ & $\begin{array}{l}\text { Application to wet foliage or during periods of } \\
\text { high humidity may cause injury to tender } \\
\text { foliage. }\end{array}$ \\
\hline \multirow[t]{4}{*}{ Chlorpyrifos } & Chlorpyrifos Pro 2 & $\mathrm{~F}$ & Nursery stock in nurseries \\
\hline & Chlorpyrifos Pro 4 & $F, G$ & $\begin{array}{l}\text { Nursery }(\mathrm{F}) \text {; soil treatment, dormant spray of } \\
\text { tree pests, and pre-plant incorporation of field } \\
\text { grown nursery stock }(\mathrm{G}) \text {; some varieties of } \\
\text { azaleas, camellias, poinsettias, rose bushes, } \\
\text { or variegated ivy show varying degrees of } \\
\text { phytotoxicity. }\end{array}$ \\
\hline & Duragard ME & $F, G$ & $\begin{array}{l}\text { Nursery (F); bedding plants, potted and cut } \\
\text { flowers. Direct spray to some open blooms } \\
\text { may cause petal drop. Do not spray on } \\
\text { kalanchoes. }\end{array}$ \\
\hline & Dursban 50W & $\mathrm{F}$ & Nursery \\
\hline Chlorpyrifos \& Cyfluthrin & Duraplex TR & $\mathrm{G}$ & \\
\hline
\end{tabular}


Table 12. Mealybug (Homoptera: Pseudococcidae) control measures available to commercial producers of any flower crop in Florida.

\begin{tabular}{|c|c|c|c|}
\hline Common Name & Trade Name/Formulation & $\begin{array}{l}\text { Production } \\
\text { Site }^{1}\end{array}$ & Notes from Label ${ }^{2}$ \\
\hline $\begin{array}{l}\text { Clarified Hydrophobic } \\
\text { Extract of Neem Oil }\end{array}$ & Triact 70 & $F, G$ & $\begin{array}{l}\text { Test before applying to carnation and the } \\
\text { flowers of: Impatiens, fuchsia, hibiscus, and } \\
\text { some roses. Most effective when applied } \\
\text { early to mid-morning or late afternoon. }\end{array}$ \\
\hline Cyfluthrin & Decathlon $20 \mathrm{WP}$ & $\mathrm{F}, \mathrm{G}$ & \\
\hline Deltamethrin & Deltagard GC 5 SC & $\mathrm{F}$ & Nursery \\
\hline Diazinon & KnoxOut NL & $\mathrm{F}$ & Citrus, stem \& root mealybugs; nursery \\
\hline Fenpropathrin & Tame 2.4EC & $\mathrm{F}, \mathrm{G}$ & $\begin{array}{l}\text { Nursery \& shade house }(\mathrm{F}) \text {; do not use less } \\
\text { than a } 7 \text { day re-treatment interval. }\end{array}$ \\
\hline \multirow[t]{3}{*}{ Imidacloprid } & Marathon $1 \%$ Granular & $F, G$ & $\begin{array}{l}\text { Nursery }(\mathrm{F}) \text {; for outdoor ornamentals grown } \\
\text { in beds, applications can not exceed a total of } \\
40 \mathrm{lbs} \text {. per acre per year. On plants with a } \\
\text { production cycle of less than } 1 \text { year, } \\
\text { application is not to exceed a frequency of } \\
\text { more than once each } 16 \text { weeks for a } \\
\text { particular plant. On stock plants and woody } \\
\text { crops with a production cycle of greater than } \\
1 \text { year, application may not exceed once a } \\
\text { year. }\end{array}$ \\
\hline & Marathon $60 \mathrm{WP}$ & $F, G$ & $\begin{array}{l}\text { Nursery }(\mathrm{F}) \text {; this product is to be applied as a } \\
\text { soil treatment only. For outdoor ornamentals } \\
\text { grown in beds applications can not exceed a } \\
\text { total of } 10.7 \text { oz. ( } 0.4 \text { lbs. active ingredient) per } \\
\text { acre per year. On plants with a production } \\
\text { cycle of less than } 1 \text { year, application is not to } \\
\text { exceed a frequency of more than once each } \\
16 \text { weeks for a particular plant. On stock } \\
\text { plants and woody crops with a production } \\
\text { cycle of greater than } 1 \text { year, application may } \\
\text { not exceed once a year. }\end{array}$ \\
\hline & Marathon II & $F, G$ & $\begin{array}{l}\text { For outdoor ornamentals do not apply more } \\
\text { than } 1.6 \text { pt. ( } 0.4 \text { lbs. a.i.) per acre per year. } \\
\text { Do not apply thru any type of irrigation } \\
\text { system. }\end{array}$ \\
\hline Lambda-cyhalothrin & Scimitar GC & $F, G$ & $\begin{array}{l}\text { Nursery \& shade house (F); do not apply } \\
\text { more than } 0.36 \text { lbs. a.i. ( } 52.4 \mathrm{fl} \text {. oz. of } \\
\text { concentrate) per acre per year. }\end{array}$ \\
\hline Malathion & $\begin{array}{l}\text { Atrapa 5E } \\
\text { Gowan Malathion } 8 \mathrm{~F} \\
\text { Malathion } 5 \mathrm{EC}\end{array}$ & $\mathrm{F}$ & \\
\hline Methidathion & Supracide $25-W$ & $\mathrm{~F}$ & Nursery stock \\
\hline
\end{tabular}


Table 12. Mealybug (Homoptera: Pseudococcidae) control measures available to commercial producers of any flower crop in Florida.

\begin{tabular}{|c|c|c|c|}
\hline Common Name & Trade Name/Formulation & $\begin{array}{l}\text { Production } \\
\text { Site }^{1}\end{array}$ & Notes from Label $^{2}$ \\
\hline Naled & Dibrom 8 Emulsive & $G$ & $\begin{array}{l}\text { Vapor treatment; spotting, bronzing or } \\
\text { localized burning can occur under certain } \\
\text { climatic conditions such as slow drying and } \\
\text { high humidity or stress caused by drought or } \\
\text { high temperature. White Butterfly and Golden } \\
\text { Rapture roses, Pink Champagne } \\
\text { chrysanthemums, green wandering jew, } \\
\text { poinsettias and Dutchman's pipe may be } \\
\text { injured by vapor. }\end{array}$ \\
\hline Permethrin & Astro & $\mathrm{G}$ & $\begin{array}{l}\text { Do not apply more than } 2.0 \mathrm{lb} \text {. a.i. per acre } \\
\text { per year. }\end{array}$ \\
\hline \multirow[t]{2}{*}{$\begin{array}{l}\text { Potassium Salts of Fatty } \\
\text { Acids }\end{array}$} & Insecticidal Soap 49.52 CF & $\mathrm{F}, \mathrm{G}$ & $\begin{array}{l}\text { Caution: Do not use on new transplants, } \\
\text { newly rooted cuttings, plants stressed by } \\
\text { drought, or when tender new foliage is } \\
\text { present. Avoid application when leaf } \\
\text { temperature exceeds } 85^{\circ} \text { F. Note: Do not } \\
\text { use on crown of thorns and test on other } \\
\text { euphorbias for phytotoxicity. Do not use on } \\
\text { bleeding heart, lantana, lilies or sweetpeas. } \\
\text { Some varieties of azaleas, begonias, } \\
\text { camellias, fuschias, gardenias, orchids, and } \\
\text { impatiens have shown sensitivity. Do not } \\
\text { apply to poinsettias after bract coloration } \\
\text { begins. Test on chrysanthemums for varietal } \\
\text { sensitivity and do not apply to open blooms. } \\
\text { Do not apply to ornamentals stressed by } \\
\text { drought. Test on eugenia and euonymus for } \\
\text { varietal and drought sensitivity. }\end{array}$ \\
\hline & M-Pede & $F, G$ & $\begin{array}{l}\text { Tank mixes with chlorothalonil should be } \\
\text { avoided. Potential for injury increases on } \\
\text { plants stressed by heat, humidity, drought, or } \\
\text { insect, mite or disease pressure. Do not apply } \\
\text { to very sensitive plants such as: Cherimoya, } \\
\text { bleeding heart, or sweetpeas. The following } \\
\text { may be sensitive: Crown of thorns and other } \\
\text { euphorbia, fuchsia, gardenia, impatiens, } \\
\text { Asiatic and oriental lily varieties, lantana, } \\
\text { ornamental ivy, poinsettia, redbud, } \\
\text { schefflera, zebra plant and some succulents. } \\
\text { Flowers of the following may be injured when } \\
\text { sprayed: African violet, ageratum, azalea, } \\
\text { begonia, camellia, chrysanthemum, dahlia, } \\
\text { geranium, gloxinia, impatiens, lily, marigold, } \\
\text { orchid, pansy, petunia, poinsettia bracts, } \\
\text { rose, salvia, snapdragon, vinca, and zinnia. }\end{array}$ \\
\hline
\end{tabular}


Table 12. Mealybug (Homoptera: Pseudococcidae) control measures available to commercial producers of any flower crop in Florida.

\begin{tabular}{|c|c|c|c|}
\hline Common Name & Trade Name/Formulation & $\begin{array}{l}\text { Production } \\
\text { Site }^{1}\end{array}$ & Notes from Label ${ }^{2}$ \\
\hline \multirow{2}{*}{$\begin{array}{l}\text { Pyrethrins \& } \\
\text { Piperonyl Butoxide }\end{array}$} & 1100 Pyrethrum TR & $\mathrm{G}$ & \\
\hline & Pyrenone Crop Spray & $F, G$ & \\
\hline $\begin{array}{l}\text { Pyrethrins, } \\
\text { Piperonyl Butoxide \& } \\
\text { Silicon Dioxide }\end{array}$ & $\begin{array}{l}\text { Diatect Multipurpose } \\
\text { Insecticide II }\end{array}$ & $\mathrm{F}$ & \\
\hline $\begin{array}{l}\text { Pyrethrins, Rotenone \& } \\
\text { other Cube Resins }\end{array}$ & Pyrellin E.C. & $F, G$ & Apply in intervals of 7 days or less. \\
\hline Pyriproxyfen & Distance IGR & $\mathrm{F}, \mathrm{G}$ & $\begin{array}{l}\text { Suppression; for foliar spray application, } \\
\text { apply Distance no more than two times per } \\
\text { cropping cycle or no more than two times per } \\
6 \text { months. For sprench application, if a } \\
\text { second application is needed, allow a } \\
\text { minimum of } 21 \text { days between applications. } \\
\text { For drench application: Do not drench plants } \\
\text { more than one time per crop cycle. } \\
\text { Phytotoxicity has been observed on salvia } \\
\text { (Salvia spp.), ghost plant (Graptopetalum } \\
\text { paraguayense), schefflera (Schefflera spp.), } \\
\text { gardenia (Gardenia spp.), and coral bells } \\
\text { (Heuchera sanguinea). It is therefore } \\
\text { recommended that Distance not be used on } \\
\text { these plants. Do not apply to poinsettia after } \\
\text { bract formation. }\end{array}$ \\
\hline \multirow[t]{2}{*}{$\begin{array}{l}\text { Refined Petroleum } \\
\text { Distillate }\end{array}$} & Saf-T-Side Spray Oil & $\mathrm{F}$ & $\begin{array}{l}\text { This insecticide's mode of action is through } \\
\text { suffocation of eggs, larvae and nymphs of } \\
\text { insects and adults of soft bodied insects. This } \\
\text { mode of action necessitates total spray } \\
\text { coverage. Bedding plants; do not use in } \\
\text { combination with or immediately before or } \\
\text { after spraying with fungicides such as captan, } \\
\text { oxythioquinox or any product containing } \\
\text { sulfur. Also do not use with carbaryl (Sevin) } \\
\text { or dimethoate. Do not use with any product } \\
\text { whose label recommends the use of no oils. } \\
\text { Do not use in combination with NPK foliar } \\
\text { fertilizer applications. }\end{array}$ \\
\hline & Ultra-Fine Oil & $\mathrm{F}$ & $\begin{array}{l}\text { Caution: Spray no more than } 4 \text { consecutive } \\
\text { sprays. The frequency of consecutive sprays } \\
\text { should not exceed once every } 2 \text { weeks. Early } \\
\text { morning applications are recommended. Do } \\
\text { not tank mix with insecticide or miticide whose } \\
\text { label indicates that it should not be used with } \\
\text { oil. Do not use with captan, chlorothalonil, } \\
\text { dimethoate, methiocarb, oxythioquinox, } \\
\text { propargite, or any product containing sulfur. } \\
\text { This list is not exhaustive; therefore for } \\
\text { products not included in this list consult label } \\
\text { for compatibility information. }\end{array}$ \\
\hline
\end{tabular}


Table 12. Mealybug (Homoptera: Pseudococcidae) control measures available to commercial producers of any flower crop in Florida.

\begin{tabular}{||l|l|l|l||}
\hline \hline \multicolumn{1}{|c|}{ Common Name } & Trade Name/Formulation & $\begin{array}{c}\text { Production } \\
\text { Site }^{1}\end{array}$ & Notes from Label \\
\hline S-Kinoprene & Enstar II & $G$ & $\begin{array}{l}\text { Application should be made to poinsettia } \\
\text { before bract formation. Foliar damage on } \\
\text { some sensitive varieties can result. Some } \\
\text { varieties of roses, such as Yellow Blooded } \\
\text { roses, show delayed damage. Slight to } \\
\text { moderate injury has occurred on some } \\
\text { blooms under certain conditions, suggest } \\
\text { application be made in pre-bloom stage. }\end{array}$ \\
\hline $\begin{array}{l}\text { Tau-Fluvalinate } \\
\text { Thiamethoxam }\end{array}$ & Mavrik Aquaflow & F, G & $\begin{array}{l}\text { Repeat applications not more than four times } \\
\text { per month at 5-7 day intervals unless } \\
\text { otherwise noted in use directions. }\end{array}$ \\
\hline $\begin{array}{l}\text { Footnotes: } \\
1 \text { "F" indicates field production. "G" indicates greenhouse production. } \\
{ }^{2} \text { Notes are taken from product labels and restrict use to the condition indicated (suppression, beet armyworm, exposed } \\
\text { thrips, etc.), limit numbers or patterns of applications, provide phytotoxicity precautions, etc. }\end{array}$ & $\begin{array}{l}\text { Shade house, container \& nursery grown (F); } \\
\text { do not exceed 8 oz. per acre per crop or year, } \\
\text { which ever is shorter. }\end{array}$ \\
\hline \hline
\end{tabular}

Table 13. Mites, broad \& cyclamen (Acari: Tarsonemidae) control measures available to commercial producers of any flower crop in Florida.

\begin{tabular}{|c|c|c|c|}
\hline Common Name & Trade Name/Formulation & $\begin{array}{l}\text { Production } \\
\text { Site }^{1}\end{array}$ & Notes from Label ${ }^{2}$ \\
\hline Abamectin & Avid $0.15 \mathrm{EC}$ & $F, G$ & $\begin{array}{l}\text { Phytotoxicity has been observed on Shasta } \\
\text { daisy. }\end{array}$ \\
\hline \multirow[t]{4}{*}{ Bifenthrin } & $\begin{array}{l}\text { Attain TR } \\
\text { Attain TR Micro }\end{array}$ & $G$ & Mites \\
\hline & $\begin{array}{l}\text { Talstar Flowable } \\
\text { Talstar GH Prescription }\end{array}$ & $G$ & Broad mites \\
\hline & Talstar N & $F, G$ & $\begin{array}{l}\text { Mites such as broad mites; nursery \& shade } \\
\text { house }(F) \text {; do not apply more than } 0.2 \text { lbs. a.i. } \\
\text { per acre per year for outdoor applications. }\end{array}$ \\
\hline & Talstar Nursery Flowable & $F, G$ & $\begin{array}{l}\text { Mites such as broad mites }(F) \text {; broad mites } \\
(\mathrm{G}) \text {; nursery \& shade house }(\mathrm{F}) \text {; do not apply } \\
\text { more than } 0.2 \text { lbs. a.i. per acre per year for } \\
\text { outdoor applications. }\end{array}$ \\
\hline
\end{tabular}


Table 13. Mites, broad \& cyclamen (Acari: Tarsonemidae) control measures available to commercial producers of any flower crop in Florida.

\begin{tabular}{|c|c|c|c|}
\hline Common Name & Trade Name/Formulation & $\begin{array}{l}\text { Production } \\
\text { Site }^{1}\end{array}$ & Notes from Label ${ }^{2}$ \\
\hline Chlorfenapyr & Pylon & $G$ & $\begin{array}{l}\text { Broad mite (Polyphagotarsonemus latus), } \\
\text { cyclamen mite (Phytonemus pallidus); } \\
\text { non-edible ornamentals; do not apply Pylon } \\
\text { more than two times consecutively or a total } \\
\text { of three times during a growing cycle (not } \\
\text { more than } 41 \text { fl. oz. of product / } 100 \text { gal. / } \\
\text { crop / season or } 0.64 \text { lbs. of a.i. / } 100 \text { gal. / } \\
\text { crop / season) (start to finish for one } \\
\text { ornamental crop). Do not apply Pylon to } \\
\text { consecutive crops in a greenhouse unless the } \\
\text { Pylon is applied in combination with other } \\
\text { effective miticides or insecticides with a } \\
\text { different mode of action. Phytotoxicity is likely } \\
\text { to occur to some varieties of carnations, } \\
\text { dianthus, kalanchoe, poinsettia, roses, salvia, } \\
\text { and zinnia. Additions of crop oils, surfactants, } \\
\text { and fertilizers or other tank additives have } \\
\text { been shown to increase the likelihood of foliar } \\
\text { injury. }\end{array}$ \\
\hline \multirow[t]{2}{*}{ Diazinon } & $\begin{array}{l}\text { Diazinon 50W } \\
\text { Diazinon AG } 500\end{array}$ & $\mathrm{~F}$ & $\begin{array}{l}\text { Cyclamen mites; precaution: Do not use on } \\
\text { ferns, poinsettias, hibiscus, pilea, and } \\
\text { gardenia since injury to the plants may occur. }\end{array}$ \\
\hline & KnoxOut NL & $\mathrm{F}$ & Mites; nursery \\
\hline Dicofol & Kelthane 50WSP & $F, G$ & $\begin{array}{l}\text { Nursery (F); applicators (applicators using } \\
\text { this product for agricultural uses only) must } \\
\text { be in enclosed cabs or cockpits. Do not make } \\
\text { more than two applications per crop or } \\
\text { season. Resistance management: As a } \\
\text { general rule, the utility of Kelthane } 50 \mathrm{WSP} \\
\text { can be maintained indefinitely by rotating use } \\
\text { of Kelthane 50WSP with miticides with } \\
\text { different modes of action and by limiting the } \\
\text { number of applications of Kelthane 50WSP } \\
\text { to an average of one per season. If two } \\
\text { applications are made in a single growing } \\
\text { season, no application should be made } \\
\text { during the following year. }\end{array}$ \\
\hline Endosulfan & $\begin{array}{l}\text { Endosulfan } 3 \text { EC } \\
\text { Endosulfan } 50 \text { WP } \\
\text { Thionex (Endosulfan) } 3 \mathrm{EC} \\
\text { Thionex (Endosulfan) } 50 \mathrm{WP}\end{array}$ & $F, G$ & $\begin{array}{l}\text { Cyclamen mite; do not exceed } 3.0 \text { lbs. a.i. per } \\
\text { acre per year. On chrysanthemums, best } \\
\text { results will be obtained if applied before } \\
\text { plants flower. }\end{array}$ \\
\hline Lambda-cyhalothrin & Scimitar GC & $F, G$ & $\begin{array}{l}\text { Mites such as broad mites }(\mathrm{F}) \text {; broad mite } \\
(\mathrm{G}) \text {; nursery } \& \text { shade house }(\mathrm{F}) \text {; do not apply } \\
\text { more than } 0.36 \mathrm{lbs} \text {. a.i. ( } 52.4 \mathrm{fl} \text {. oz. of } \\
\text { concentrate) per acre per year. }\end{array}$ \\
\hline Pyridaben & Sanmite & $F, G$ & $\begin{array}{l}\text { Broad mite; maximum seasonal use rate: Do } \\
\text { not exceed } 21.34 \text { ounces per acre per year. }\end{array}$ \\
\hline
\end{tabular}


Table 13. Mites, broad \& cyclamen (Acari: Tarsonemidae) control measures available to commercial producers of any flower crop in Florida.

\begin{tabular}{||l|l|l|l||}
\hline \hline \multicolumn{1}{|c|}{ Common Name } & Trade Name/Formulation & $\begin{array}{c}\text { Production } \\
\text { Site }^{1}\end{array}$ & \multicolumn{1}{|c||}{ Notes from Label $^{2}$} \\
\hline $\begin{array}{l}\text { Refined Petroleum } \\
\text { Distillate }\end{array}$ & Saf-T-Side Spray Oil & F & $\begin{array}{l}\text { This insecticide's mode of action is through } \\
\text { suffocation of eggs, larvae and nymphs of } \\
\text { insects and adults of soft bodied insects. This } \\
\text { mode of action necessitates total spray } \\
\text { coverage. Bedding plants; do not use in } \\
\text { combination with or immediately before or } \\
\text { after spraying with fungicides such as captan, } \\
\text { oxythioquinox or any product containing } \\
\text { sulfur. Also do not use with carbaryl (Sevin) } \\
\text { or dimethoate. Do not use with any product } \\
\text { whose label recommends the use of no oils. } \\
\text { Do not use in combination with NPK foliar } \\
\text { fertilizer applications. }\end{array}$ \\
\hline $\begin{array}{l}\text { Footnotes: } \\
1 \text { "F" indicates field production. "G" indicates greenhouse production. } \\
2 \text { Notes are taken from product labels and restrict use to the condition indicated (suppression, beet armyworm, exposed } \\
\text { thrips, etc.), limit numbers or patterns of applications, provide phytotoxicity precautions, etc. }\end{array}$ \\
\hline \hline
\end{tabular}

Table 14. Mites, eriophyid (Acari: Eriophyidae) control measures available to commercial producers of any flower crop in Florida.

\begin{tabular}{||l|l|l|l||}
\hline \hline \multicolumn{1}{|c|}{ Common Name } & Trade Name/Formulation & $\begin{array}{c}\text { Production } \\
\text { Site }^{1}\end{array}$ & \multicolumn{1}{||}{ Notes from Label $^{2}$} \\
\hline Abamectin & Avid 0.15 EC & $\mathrm{F}, \mathrm{G}$ & $\begin{array}{l}\text { Bud \& rust mites; phytotoxicity has been } \\
\text { observed on Shasta daisy. }\end{array}$ \\
\hline Carbaryl & $\begin{array}{l}\text { Carbaryl 4L } \\
\text { Carbaryl 80S }\end{array}$ & $\mathrm{F}$ & $\begin{array}{l}\text { Application to wet foliage or during periods } \\
\text { of high humidity may cause injury to tender } \\
\text { foliage. }\end{array}$ \\
\hline
\end{tabular}


Table 14. Mites, eriophyid (Acari: Eriophyidae) control measures available to commercial producers of any flower crop in Florida.

\begin{tabular}{|c|c|c|c|}
\hline Common Name & Trade Name/Formulation & $\begin{array}{l}\text { Production } \\
\text { Site }^{1}\end{array}$ & Notes from Label ${ }^{2}$ \\
\hline Chlorfenapyr & Pylon & $\mathrm{G}$ & $\begin{array}{l}\text { Citrus budmite (Eriophyes sheldoni), rust } \\
\text { mite (Phyllocoptruta sp., Epitrimerus sp., } \\
\text { Aculus sp.); non-edible ornamentals; do not } \\
\text { apply Pylon more than two times } \\
\text { consecutively or a total of three times during } \\
\text { a growing cycle (not more than } 41 \text { fl. oz. of } \\
\text { product / } 100 \text { gal. / crop / season or } 0.64 \text { lbs. } \\
\text { of a.i. / } 100 \text { gal. / crop / season) (start to } \\
\text { finish for one ornamental crop). Do not } \\
\text { apply Pylon to consecutive crops in a } \\
\text { greenhouse unless the Pylon is applied in } \\
\text { combination with other effective miticides or } \\
\text { insecticides with a different mode of action. } \\
\text { Phytotoxicity is likely to occur to some } \\
\text { varieties of carnations, dianthus, kalanchoe, } \\
\text { poinsettia, roses, salvia, and zinnia. } \\
\text { Additions of crop oils, surfactants, and } \\
\text { fertilizers or other tank additives have been } \\
\text { shown to increase the likelihood of foliar } \\
\text { injury. }\end{array}$ \\
\hline \multirow[t]{3}{*}{ Chlorpyrifos } & Chlorpyrifos Pro 2 & $\mathrm{~F}$ & $\begin{array}{l}\text { Mites such as bermudagrass; nursery stock } \\
\text { in nurseries }\end{array}$ \\
\hline & Chlorpyrifos Pro 4 & $F, G$ & $\begin{array}{l}\text { Mites such as bermudagrass; nursery }(\mathrm{F}) \text {; } \\
\text { soil treatment, dormant spray of tree pests, } \\
\text { and pre-plant incorporation of field grown } \\
\text { nursery stock }(\mathrm{G}) \text {; some varieties of } \\
\text { azaleas, camellias, poinsettias, rose } \\
\text { bushes, or variegated ivy show varying } \\
\text { degrees of phytotoxicity. }\end{array}$ \\
\hline & Dursban 50W & $\mathrm{F}$ & Mites; nursery \\
\hline \multirow[t]{2}{*}{ Diazinon } & $\begin{array}{l}\text { Diazinon 50W } \\
\text { Diazinon AG } 500\end{array}$ & $\mathrm{~F}$ & $\begin{array}{l}\text { Carnation bud mites, carnation shoot mites; } \\
\text { precaution: Do not use on ferns, poinsettias, } \\
\text { hibiscus, pilea, and gardenia since injury to } \\
\text { the plants may occur. }\end{array}$ \\
\hline & KnoxOut NL & $\mathrm{F}$ & Mites; nursery \\
\hline
\end{tabular}


Table 14. Mites, eriophyid (Acari: Eriophyidae) control measures available to commercial producers of any flower crop in Florida.

\begin{tabular}{|c|c|c|c|}
\hline Common Name & Trade Name/Formulation & $\begin{array}{l}\text { Production } \\
\text { Site }^{1}\end{array}$ & Notes from Label $^{2}$ \\
\hline Dicofol & Kelthane 50WSP & $F, G$ & $\begin{array}{l}\text { Pear rust mite, plum nursery mite, plum rust } \\
\text { mite, tomato russet mite; nursery (F); } \\
\text { applicators (applicators using this product } \\
\text { for agricultural uses only) must be in } \\
\text { enclosed cabs or cockpits. Do not make } \\
\text { more than two applications per crop or } \\
\text { season. Resistance management: As a } \\
\text { general rule, the utility of Kelthane 50WSP } \\
\text { can be maintained indefinitely by rotating } \\
\text { use of Kelthane 50WSP with miticides with } \\
\text { different modes of action and by limiting the } \\
\text { number of applications of Kelthane } 50 W S P \\
\text { to an average of one per season. If two } \\
\text { applications are made in a single growing } \\
\text { season, no application should be made } \\
\text { during the following year. }\end{array}$ \\
\hline Endosulfan & $\begin{array}{l}\text { Endosulfan } 3 \text { EC } \\
\text { Endosulfan } 50 \text { WP } \\
\text { Thionex (Endosulfan) } 3 \text { EC } \\
\text { Thionex (Endosulfan) } 50 \\
\end{array}$ & $\mathrm{~F}$ & $\begin{array}{l}\text { Taxus bud mite; do not exceed } 3.0 \text { lbs. a.i. } \\
\text { per acre per year. On chrysanthemums, } \\
\text { best results will be obtained if applied before } \\
\text { plants flower. }\end{array}$ \\
\hline $\begin{array}{l}\text { Potassium Salts of Fatty } \\
\text { Acids }\end{array}$ & MNPede & $F, G$ & $\begin{array}{l}\text { Mites such as rust mite }(F) \text {; rust mite }(G) ; \\
\text { tank mixes with chlorothalonil should be } \\
\text { avoided. Potential for injury increases on } \\
\text { plants stressed by heat, humidity, drought, } \\
\text { or insect, mite or disease pressure. Do not } \\
\text { apply to very sensitive plants such as: } \\
\text { Cherimoya, bleeding heart, or sweetpeas. } \\
\text { The following may be sensitive: Crown of } \\
\text { thorns and other euphorbia, fuchsia, } \\
\text { gardenia, impatiens, Asiatic and oriental lily } \\
\text { varieties, lantana, ornamental ivy, } \\
\text { poinsettia, redbud, schefflera, zebra plant } \\
\text { and some succulents. Flowers of the } \\
\text { following may be injured when sprayed: } \\
\text { African violet, ageratum, azalea, begonia, } \\
\text { camellia, chrysanthemum, dahlia, geranium, } \\
\text { gloxinia, impatiens, lily, marigold, orchid, } \\
\text { pansy, petunia, poinsettia bracts, rose, } \\
\text { salvia, snapdragon, vinca, and zinnia. }\end{array}$ \\
\hline
\end{tabular}


Table 14. Mites, eriophyid (Acari: Eriophyidae) control measures available to commercial producers of any flower crop in Florida.

\begin{tabular}{|c|c|c|c|}
\hline Common Name & Trade Name/Formulation & $\begin{array}{l}\text { Production } \\
\text { Site }^{1}\end{array}$ & Notes from Label $^{2}$ \\
\hline \multirow[t]{2}{*}{$\begin{array}{l}\text { Refined Petroleum } \\
\text { Distillate }\end{array}$} & Saf-T-Side Spray Oil & $\mathrm{F}$ & $\begin{array}{l}\text { This insecticide's mode of action is through } \\
\text { suffocation of eggs, larvae and nymphs of } \\
\text { insects and adults of soft bodied insects. } \\
\text { This mode of action necessitates total spray } \\
\text { coverage. Bedding plants; do not use in } \\
\text { combination with or immediately before or } \\
\text { after spraying with fungicides such as } \\
\text { captan, oxythioquinox or any product } \\
\text { containing sulfur. Also do not use with } \\
\text { carbaryl (Sevin) or dimethoate. Do not use } \\
\text { with any product whose label recommends } \\
\text { the use of no oils. Do not use in combination } \\
\text { with NPK foliar fertilizer applications. }\end{array}$ \\
\hline & Ultra-Fine Oil & $\mathrm{F}$ & $\begin{array}{l}\text { Caution: Spray no more than } 4 \text { consecutive } \\
\text { sprays. The frequency of consecutive } \\
\text { sprays should not exceed once every } 2 \\
\text { weeks. Early morning applications are } \\
\text { recommended. Do not tank mix with } \\
\text { insecticide or miticide whose label indicates } \\
\text { that it should not be used with oil. Do not } \\
\text { use with captan, chlorothalonil, dimethoate, } \\
\text { methiocarb, oxythioquinox, propargite, or } \\
\text { any product containing sulfur. This list is not } \\
\text { exhaustive; therefore for products not } \\
\text { included in this list consult label for } \\
\text { compatibility information. }\end{array}$ \\
\hline \multicolumn{4}{|c|}{$\begin{array}{l}\text { Footnotes: } \\
1 \text { "F" indicates field production. "G" indicates greenhouse production. } \\
{ }^{2} \text { Notes are taken from product labels and restrict use to the condition indicated (suppression, beet armyworm, exposed } \\
\text { thrips, etc.), limit numbers or patterns of applications, provide phytotoxicity precautions, etc. }\end{array}$} \\
\hline
\end{tabular}

Table 15. Mites, privet (Acari: Tenuipalpidae (false spider mites)) control measures available to commercial producers of any flower crop in Florida.

\begin{tabular}{||l|l|l|l||}
\hline \hline \multicolumn{1}{||c|}{ Common Name } & \multicolumn{1}{|c||}{ Trade Name/Formulation } & $\begin{array}{c}\text { Production } \\
\text { Site }^{1}\end{array}$ & \multicolumn{1}{|c||}{ Notes from Label $^{2}$} \\
\hline Diazinon & $\begin{array}{l}\text { Diazinon 50W } \\
\text { Diazinon AG 500 }\end{array}$ & $\mathrm{F}$ & $\begin{array}{l}\text { Privet mites; precaution: Do not use on ferns, } \\
\text { poinsettias, hibiscus, pilea, and gardenia } \\
\text { since injury to the plants may occur. }\end{array}$ \\
\cline { 2 - 4 } & KnoxOut NL & F & Mites; nursery \\
\hline \hline
\end{tabular}


Table 15. Mites, privet (Acari: Tenuipalpidae (false spider mites)) control measures available to commercial producers of any flower crop in Florida.

\begin{tabular}{|c|c|c|c|}
\hline Common Name & Trade Name/Formulation & $\begin{array}{l}\text { Production } \\
\text { Site }^{1}\end{array}$ & Notes from Label ${ }^{2}$ \\
\hline Dicofol & Kelthane 50WSP & $F, G$ & $\begin{array}{l}\text { Privet mites; nursery (F); applicators } \\
\text { (applicators using this product for agricultural } \\
\text { uses only) must be in enclosed cabs or } \\
\text { cockpits. Do not make more than two } \\
\text { applications per crop or season. Resistance } \\
\text { management: As a general rule, the utility of } \\
\text { Kelthane } 50 \mathrm{WSP} \text { can be maintained } \\
\text { indefinitely by rotating use of Kelthane } \\
50 W S P \text { with miticides with different modes of } \\
\text { action and by limiting the number of } \\
\text { applications of Kelthane } 50 \mathrm{WSP} \text { to an } \\
\text { average of one per season. If two } \\
\text { applications are made in a single growing } \\
\text { season, no application should be made } \\
\text { during the following year. }\end{array}$ \\
\hline \multicolumn{4}{|c|}{$\begin{array}{l}\text { Footnotes: } \\
1 \text { "F" indicates field production. "G" indicates greenhouse production. } \\
{ }^{2} \text { Notes are taken from product labels and restrict use to the condition indicated (suppression, beet armyworm, exposed } \\
\text { thrips, etc.), limit numbers or patterns of applications, provide phytotoxicity precautions, etc. }\end{array}$} \\
\hline
\end{tabular}

Table 16. Mites, spider (Acari: Tetranychidae) control measures available to commercial producers of any flower crop in Florida.

\begin{tabular}{|c|c|c|c|}
\hline Common Name & Trade Name/Formulation & $\begin{array}{l}\text { Production } \\
\text { Site }^{1}\end{array}$ & Notes from Label ${ }^{2}$ \\
\hline Abamectin & Avid $0.15 \mathrm{EC}$ & $F, G$ & $\begin{array}{l}\text { Carmine spider mite, European red mite, } \\
\text { twospotted spider mite; phytotoxicity has } \\
\text { been observed on Shasta daisy. }\end{array}$ \\
\hline Acephate $^{3}$ & 1300 Orthene TR & $G$ & \\
\hline $\begin{array}{l}\text { Acephate \& } \\
\text { Fenpropathrin }^{3}\end{array}$ & Tame/Orthene TR & $\mathrm{G}$ & $\begin{array}{l}\text { Do not apply within } 48 \text { hours of a previous } \\
\text { application. }\end{array}$ \\
\hline $\begin{array}{l}\text { Beauveria bassiana } \\
\text { ATCC } 74040\end{array}$ & Naturalis L & $F, G$ & $\begin{array}{l}\text { Mites; do not tank mix with fungicides. Wait a } \\
\text { minimum of } 48 \text { hours after application before } \\
\text { applying fungicides. }\end{array}$ \\
\hline
\end{tabular}


Table 16. Mites, spider (Acari: Tetranychidae) control measures available to commercial producers of any flower crop in Florida.

\begin{tabular}{|c|c|c|c|}
\hline Common Name & Trade Name/Formulation & $\begin{array}{l}\text { Production } \\
\text { Site }^{1}\end{array}$ & Notes from Label ${ }^{2}$ \\
\hline \multirow[t]{2}{*}{ Bifenazate } & Floramite & $F, G$ & $\begin{array}{l}\text { Do not make more than two applications per } \\
\text { crop per year. Do not apply more than } 16 \text { oz. } \\
\text { per acre per year. Apply only one application } \\
\text { of Floramite before rotating to products of an } \\
\text { alternative chemical class and use at least } \\
\text { two alternative products between treatments } \\
\text { of Floramite. }\end{array}$ \\
\hline & Floramite SC & $F, G$ & $\begin{array}{l}\text { Twospotted spider mite, European red mite, } \\
\text { citrus red mite, southern red mite; do not use } \\
\text { in successive applications. Apply only one } \\
\text { application before rotating to products of an } \\
\text { alternative chemical class. Use at least two } \\
\text { alternative products between treatments of } \\
\text { Floramite SC. Do not make more than two } \\
\text { applications per crop per year. Do not apply } \\
\text { more than } 32 \mathrm{fl} \text {. oz. per acre per year. }\end{array}$ \\
\hline \multirow[t]{4}{*}{ Bifenthrin $^{3}$} & $\begin{array}{l}\text { Attain TR } \\
\text { Attain TR Micro }\end{array}$ & $G$ & Mites \\
\hline & $\begin{array}{l}\text { Talstar Flowable } \\
\text { Talstar GH Prescription }\end{array}$ & $\mathrm{G}$ & \\
\hline & Talstar N & $F, G$ & $\begin{array}{l}\text { Mites such as spider mites; nursery \& shade } \\
\text { house (F); do not apply more than } 0.2 \text { lbs. a.i. } \\
\text { per acre per year for outdoor applications. }\end{array}$ \\
\hline & Talstar Nursery Flowable & $F, G$ & $\begin{array}{l}\text { Mites such as spider mites }(\mathrm{F}) \text {; nursery \& } \\
\text { shade house }(\mathrm{F}) \text {; do not apply more than } 0.2 \\
\text { lbs. a.i. per acre per year for outdoor } \\
\text { applications. }\end{array}$ \\
\hline Chlorfenapyr & Pylon & G & $\begin{array}{l}\text { Non-edible ornamentals; do not apply Pylon } \\
\text { more than two times consecutively or a total } \\
\text { of three times during a growing cycle (not } \\
\text { more than } 41 \mathrm{fl} \text {. oz. of product/100 gal. / crop } \\
\text { / season or } 0.64 \text { lbs. of a.i. / } 100 \text { gal. / crop / } \\
\text { season) (start to finish for one ornamental } \\
\text { crop). Do not apply Pylon to consecutive } \\
\text { crops in a greenhouse unless the Pylon is } \\
\text { applied in combination with other effective } \\
\text { miticides or insecticides with a different mode } \\
\text { of action. Phytotoxicity is likely to occur to } \\
\text { some varieties of carnations, dianthus, } \\
\text { kalanchoe, poinsettia, roses, salvia, and } \\
\text { zinnia. Additions of crop oils, surfactants, and } \\
\text { fertilizers or other tank additives have been } \\
\text { shown to increase the likelihood of foliar } \\
\text { injury. }\end{array}$ \\
\hline Chlorpyrifos \& Cyfluthrin $^{3}$ & Duraplex TR & $\mathrm{G}$ & Mites \\
\hline
\end{tabular}


Table 16. Mites, spider (Acari: Tetranychidae) control measures available to commercial producers of any flower crop in Florida.

\begin{tabular}{|c|c|c|c|}
\hline Common Name & Trade Name/Formulation & $\begin{array}{l}\text { Production } \\
\text { Site }^{1}\end{array}$ & Notes from Label ${ }^{2}$ \\
\hline $\begin{array}{l}\text { Clarified Hydrophobic } \\
\text { Extract of Neem Oil }\end{array}$ & Triact 70 & $F, G$ & $\begin{array}{l}\text { Mites; test before applying to carnation and } \\
\text { the flowers of: Impatiens, fuchsia, hibiscus, } \\
\text { and some roses. Most effective when applied } \\
\text { early to mid-morning or late afternoon. }\end{array}$ \\
\hline Clofentezine & Ovation SC & $F, G$ & $\begin{array}{l}\text { Shade house, container, or nursery grown } \\
\text { (F); European red mite, twospotted spider } \\
\text { mite; recommended application frequency is } \\
\text { once per crop cycle in greenhouse, saran } \\
\text { house, and shade house culture, and once } \\
\text { per each crop cycle occurring within a year } \\
\text { for outdoor ornamentals. Has limited effect on } \\
\text { beneficial insects, particularly predatory mites } \\
\text { and can be used in ipm programs employing } \\
\text { beneficial insects. Highly active on mite eggs } \\
\text { and early mite stages. Has shown activity and } \\
\text { persistence up to } 45 \text { days after application. }\end{array}$ \\
\hline Dicofol & Kelthane 50WSP & $F, G$ & $\begin{array}{l}\text { European red mite, six-spotted mite, } \\
\text { twospotted spider mite; nursery (F); } \\
\text { applicators (applicators using this product for } \\
\text { agricultural uses only) must be in enclosed } \\
\text { cabs or cockpits. Do not make more than two } \\
\text { applications per crop or season. Resistance } \\
\text { management: As a general rule, the utility of } \\
\text { Kelthane } 50 \text { WSP can be maintained } \\
\text { indefinitely by rotating use of Kelthane } \\
50 W S P \text { with miticides with different modes of } \\
\text { action and by limiting the number of } \\
\text { applications of Kelthane } 50 W S P \text { to an } \\
\text { average of one per season. If two } \\
\text { applications are made in a single growing } \\
\text { season, no application should be made } \\
\text { during the following year. }\end{array}$ \\
\hline Etoxazole & Tetrasan 5 WDG & $F, G$ & Shade house only $(F)$ \\
\hline Fenbutatin-oxide & Vendex 50WP & $F, G$ & $\begin{array}{l}\text { Oligonychus mites, twospotted spider mite; } \\
\text { apply to foliage only, on chrysanthemums } \\
\text { (pre-bloom) and poinsettias (pre-bract). Minor } \\
\text { sensitivity observed on: Celosia argentea } \\
\text { (cockscomb), Verbenea hortensis (verbena), } \\
\text { Ageratum houstonianum (floss flower), Rosa } \\
\text { sp. (common rose - some varieties, especially } \\
\text { Yellow). When daily temperatures at } \\
\text { application averages below } 70^{\circ} \text { performance } \\
\text { is reduced. }\end{array}$ \\
\hline Fenpropathrin $^{3}$ & Tame 2.4EC & $F, G$ & $\begin{array}{l}\text { Southern red mite, twospotted spider mite; } \\
\text { nursery \& shade house }(F) \text {; do not use less } \\
\text { than a } 7 \text { day re-treatment interval. }\end{array}$ \\
\hline
\end{tabular}


Table 16. Mites, spider (Acari: Tetranychidae) control measures available to commercial producers of any flower crop in Florida.

\begin{tabular}{|c|c|c|c|}
\hline Common Name & Trade Name/Formulation & $\begin{array}{l}\text { Production } \\
\text { Site }^{1}\end{array}$ & Notes from Label $^{2}$ \\
\hline Fenpyroximate & Akari 5SC & $G$ & $\begin{array}{l}\text { Do not use in successive miticide } \\
\text { applications in the same greenhouse. Allow } \\
\text { at least two different chemicals with two } \\
\text { different modes of action to be used between } \\
\text { treatments of Akari } 5 S C \text {. Do not apply more } \\
\text { than } 48 \text { fl. oz. per crop cycle or per growing } \\
\text { season, whichever is longer. For best results } \\
\text { make no more than two applications per } \\
\text { growing season or crop cycle, whichever is } \\
\text { longer. }\end{array}$ \\
\hline \multirow[t]{2}{*}{ Hexythiazox } & Hexygon & $F, G$ & $\begin{array}{l}\text { Nursery }(\mathrm{F}) \text {; European red mite, southern red } \\
\text { mite, twospotted spider mite; for use on } \\
\text { orchids follow supplemental labeling. Make } \\
\text { only one application per crop cycle or once } \\
\text { per year. }\end{array}$ \\
\hline & Hexygon DF & $F, G$ & $\begin{array}{l}\text { Nursery (F); European red mite, southern red } \\
\text { mite, twospotted spider mite; for use on } \\
\text { orchids follow supplemental labeling. Do not } \\
\text { use Plyac or household detergents in tank } \\
\text { mix combinations with Hexygon DF. Apply } \\
\text { Hexygon DF only once per crop cycle. }\end{array}$ \\
\hline Lambda-cyhalothrin $^{3}$ & Scimitar GC & $F, G$ & $\begin{array}{l}\text { Mites such as spider mites }(\mathrm{F}) \text {; nursery \& } \\
\text { shade house }(\mathrm{F}) \text {; do not apply more than } \\
0.36 \text { lbs. a.i. ( } 52.4 \mathrm{fl} \text {. oz. of concentrate) per } \\
\text { acre per year. }\end{array}$ \\
\hline Methiocarb & Mesurol 75-W & $F, G$ & Mites; nursery (F); do not apply with oil. \\
\hline
\end{tabular}


Table 16. Mites, spider (Acari: Tetranychidae) control measures available to commercial producers of any flower crop in Florida.

\begin{tabular}{|c|c|c|c|}
\hline Common Name & Trade Name/Formulation & $\begin{array}{l}\text { Production } \\
\text { Site }^{1}\end{array}$ & Notes from Label ${ }^{2}$ \\
\hline \multirow[t]{2}{*}{$\begin{array}{l}\text { Potassium Salts of Fatty } \\
\text { Acids }\end{array}$} & Insecticidal Soap $49.52 \mathrm{CF}$ & $F, G$ & $\begin{array}{l}\text { Caution: Do not use on new transplants, } \\
\text { newly rooted cuttings, plants stressed by } \\
\text { drought, or when tender new foliage is } \\
\text { present. Avoid application when leaf } \\
\text { temperature exceeds } 85^{\circ} \mathrm{F} \text {. Note: Do not } \\
\text { use on crown of thorns and test on other } \\
\text { euphorbias for phytotoxicity. Do not use on } \\
\text { bleeding heart, lantana, lilies or sweetpeas. } \\
\text { Some varieties of azaleas, begonias, } \\
\text { camellias, fuschias, gardenias, orchids, and } \\
\text { impatiens have shown sensitivity. Do not } \\
\text { apply to poinsettias after bract coloration } \\
\text { begins. Test on chrysanthemums for varietal } \\
\text { sensitivity and do not apply to open blooms. } \\
\text { Do not apply to ornamentals stressed by } \\
\text { drought. Test on eugenia and euonymus for } \\
\text { varietal and drought sensitivity. }\end{array}$ \\
\hline & M-Pede & $F, G$ & $\begin{array}{l}\text { Mites such as spider mite (F); Tank mixes } \\
\text { with chlorothalonil should be avoided. } \\
\text { Potential for injury increases on plants } \\
\text { stressed by heat, humidity, drought, or insect, } \\
\text { mite or disease pressure. Do not apply to } \\
\text { very sensitive plants such as: Cherimoya, } \\
\text { bleeding heart, or sweetpeas. The following } \\
\text { may be sensitive: Crown of thorns and other } \\
\text { euphorbia, fuchsia, gardenia, impatiens, } \\
\text { Asiatic and oriental lily varieties, lantana, } \\
\text { ornamental ivy, poinsettia, redbud, } \\
\text { schefflera, zebra plant and some succulents. } \\
\text { Flowers of the following may be injured when } \\
\text { sprayed: African violet, ageratum, azalea, } \\
\text { begonia, camellia, chrysanthemum, dahlia, } \\
\text { geranium, gloxinia, impatiens, lily, marigold, } \\
\text { orchid, pansy, petunia, poinsettia bracts, } \\
\text { rose, salvia, snapdragon, vinca, and zinnia. }\end{array}$ \\
\hline Propargite & Ornamite & $\mathrm{F}$ & Do not use in spray mixes containing oil. \\
\hline $\begin{array}{l}\text { Pyrethrins \& } \\
\text { Piperonyl Butoxide }\end{array}$ & 1100 Pyrethrum TR & $\mathrm{G}$ & Mites, such as spider mites. \\
\hline $\begin{array}{l}\text { Pyrethrins, } \\
\text { Piperonyl Butoxide \& } \\
\text { Silicon Dioxide }\end{array}$ & $\begin{array}{l}\text { Diatect Multipurpose } \\
\text { Insecticide II }\end{array}$ & $\mathrm{F}$ & Mites \\
\hline $\begin{array}{l}\text { Pyrethrins, Rotenone \& } \\
\text { other Cube Resins }\end{array}$ & Pyrellin E.C. & $F, G$ & Mites; apply in intervals of 7 days or less. \\
\hline Pyridaben & Sanmite & $F, G$ & $\begin{array}{l}\text { European red mite, southern red mite, tumid } \\
\text { mite, twospotted spider mite; maximum } \\
\text { seasonal use rate: Do not exceed } 21.34 \\
\text { ounces per acre per year. }\end{array}$ \\
\hline
\end{tabular}


Table 16. Mites, spider (Acari: Tetranychidae) control measures available to commercial producers of any flower crop in Florida.

\begin{tabular}{|c|c|c|c|}
\hline Common Name & Trade Name/Formulation & $\begin{array}{l}\text { Production } \\
\text { Site }^{1}\end{array}$ & Notes from Label $^{2}$ \\
\hline \multirow[t]{2}{*}{$\begin{array}{l}\text { Refined Petroleum } \\
\text { Distillate }\end{array}$} & Saf-T-Side Spray Oil & $\mathrm{F}$ & $\begin{array}{l}\text { This insecticide's mode of action is through } \\
\text { suffocation of eggs, larvae and nymphs of } \\
\text { insects and adults of soft bodied insects. This } \\
\text { mode of action necessitates total spray } \\
\text { coverage. Bedding plants; do not use in } \\
\text { combination with or immediately before or } \\
\text { after spraying with fungicides such as captan, } \\
\text { oxythioquinox or any product containing } \\
\text { sulfur. Also do not use with carbaryl (Sevin) } \\
\text { or dimethoate. Do not use with any product } \\
\text { whose label recommends the use of no oils. } \\
\text { Do not use in combination with NPK foliar } \\
\text { fertilizer applications. }\end{array}$ \\
\hline & Ultra-Fine Oil & $F$ & $\begin{array}{l}\text { Caution: Spray no more than } 4 \text { consecutive } \\
\text { sprays. The frequency of consecutive sprays } \\
\text { should not exceed once every } 2 \text { weeks. Early } \\
\text { morning applications are recommended. Do } \\
\text { not tank mix with insecticide or miticide whose } \\
\text { label indicates that it should not be used with } \\
\text { oil. Do not use with captan, chlorothalonil, } \\
\text { dimethoate, methiocarb, oxythioquinox, } \\
\text { propargite, or any product containing sulfur. } \\
\text { This list is not exhaustive; therefore for } \\
\text { products not included in this list consult label } \\
\text { for compatibility information. }\end{array}$ \\
\hline Spinosad & Conserve SC & $F, G$ & $\begin{array}{l}\text { For spider mites, label says control is variable } \\
\text { and that a non-ionic adjuvant may increase } \\
\text { efficacy. When used in greenhouse or areas } \\
\text { of commercial production of herbaceous } \\
\text { (non-woody) ornamentals in nurseries } \\
\text { (including plant propagation beds): Do not } \\
\text { apply more than } 10 \text { times in a 12-month } \\
\text { period per crop regardless of the pest being } \\
\text { treated. Because generations of a specific } \\
\text { pest may overlap, rotate control products and } \\
\text { never apply more than three consecutive } \\
\text { applications of Conserve SC or products } \\
\text { containing the same a.i. or products with the } \\
\text { same mode of action. Except for } \\
\text { greenhouses and structures that can be } \\
\text { altered to be closed or open, do not reapply } \\
\text { within less than } 7 \text { days. }\end{array}$ \\
\hline Tau-Fluvalinate $^{3}$ & Mavrik Aquaflow & $F, G$ & $\begin{array}{l}\text { Mites such as twospotted spider mites; } \\
\text { repeat applications not more than four times } \\
\text { per month at 5-7 day intervals unless } \\
\text { otherwise noted in use directions. }\end{array}$ \\
\hline
\end{tabular}


Table 16. Mites, spider (Acari: Tetranychidae) control measures available to commercial producers of any flower crop in Florida.

\begin{tabular}{|l|c|c||}
\hline \hline \multicolumn{1}{|c|}{ Common Name } & Trade Name/Formulation & $\begin{array}{c}\text { Production } \\
\text { Site }\end{array}$ \\
\hline Footnotes: & Notes from Label $^{2}$ \\
1 "F" indicates field production. "G" indicates greenhouse production. \\
${ }^{2}$ Notes are taken from product labels and restrict use to the condition indicated (suppression, beet armyworm, exposed \\
thrips, etc.), limit numbers or patterns of applications, provide phytotoxicity precautions, etc. \\
${ }^{3}$ Some spider mite populations have become resistant to organophosphate and pyrethroid insecticides. Infrequent use \\
may be effective but repeated use may be ineffective, depending on the history of the target population. \\
\hline \hline
\end{tabular}

Table 17. Plant (lygus) bugs (Hemiptera: Miridae) control measures available to commercial producers of any flower crop in Florida.

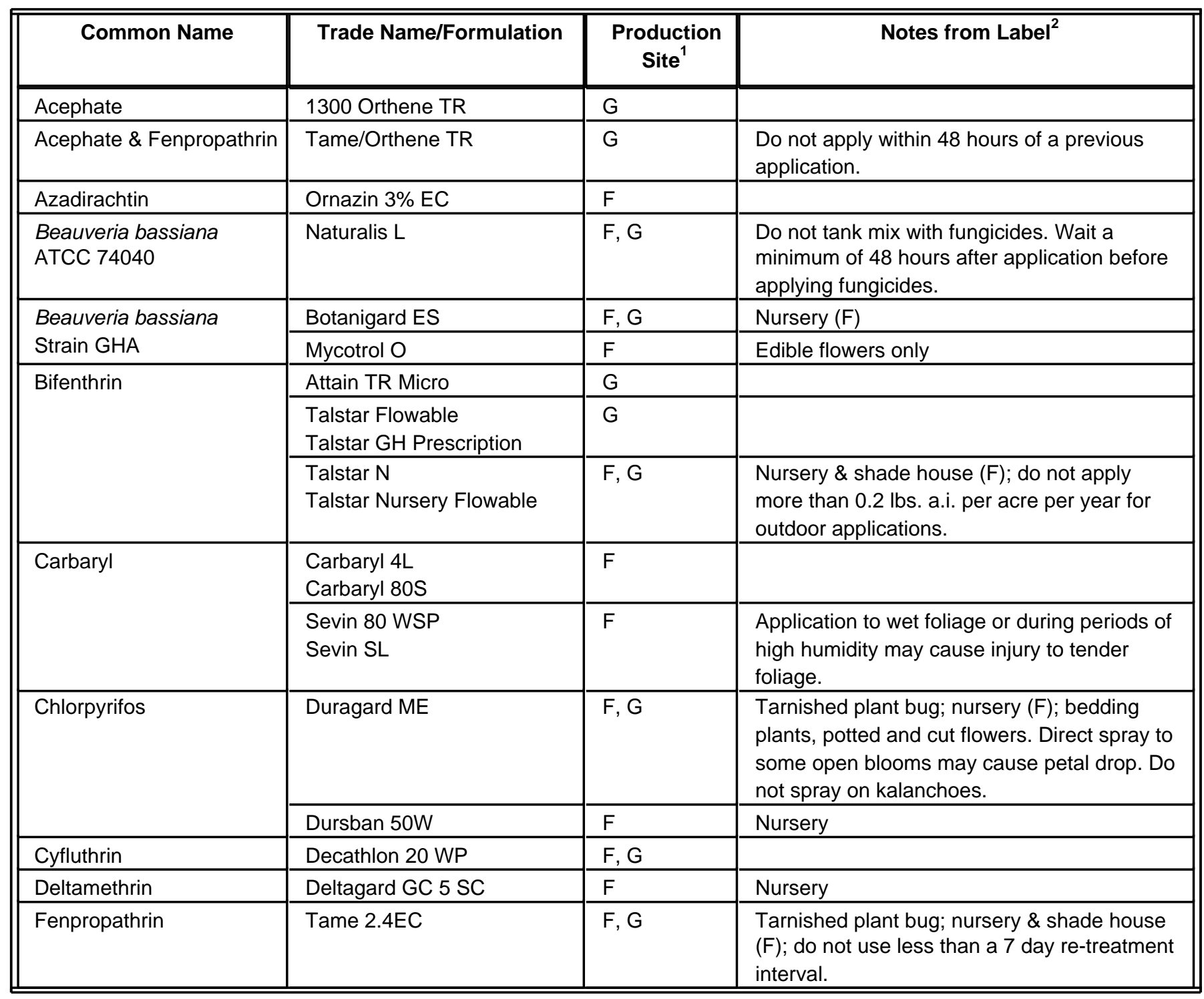


Table 17. Plant (lygus) bugs (Hemiptera: Miridae) control measures available to commercial producers of any flower crop in Florida.

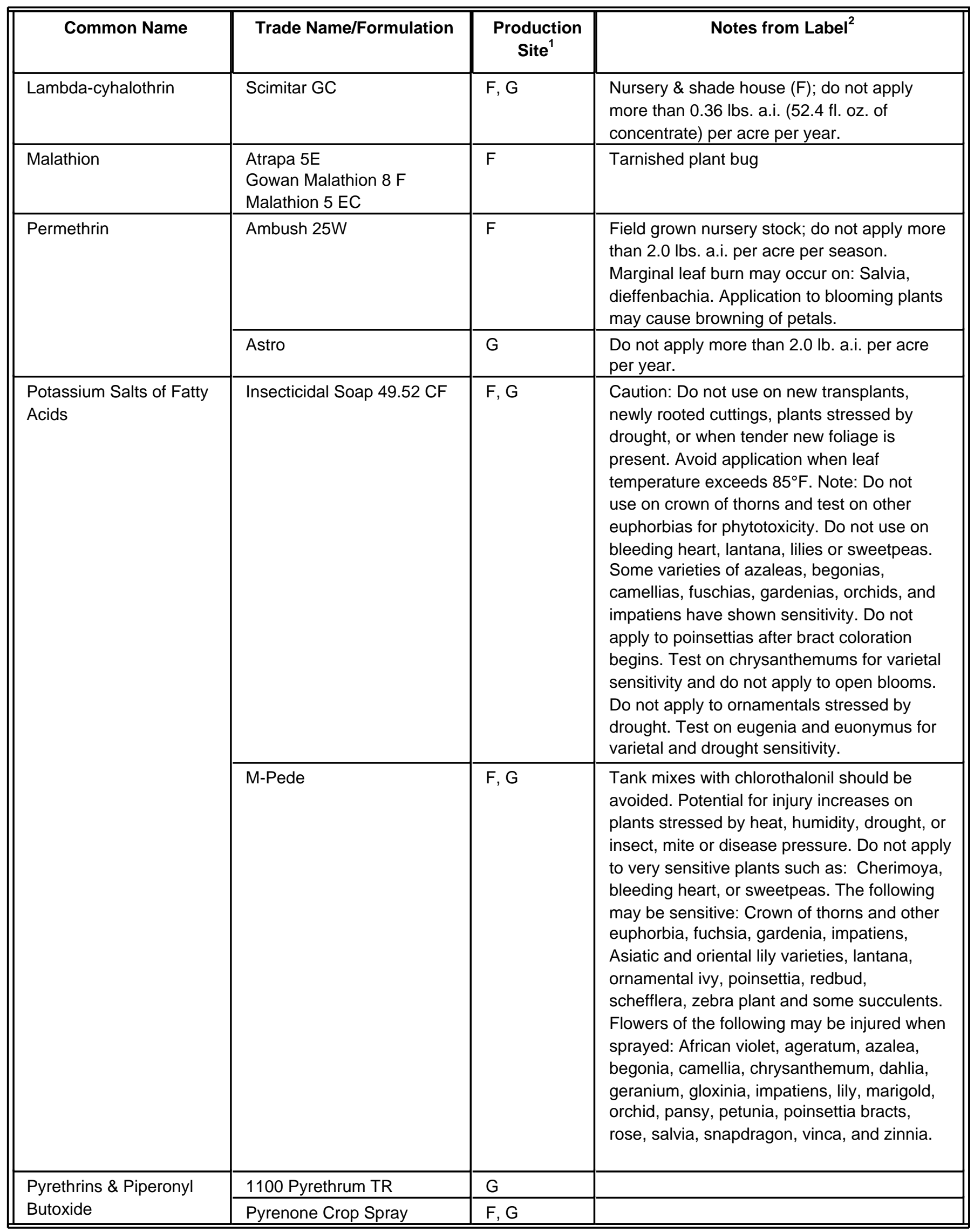


Table 17. Plant (lygus) bugs (Hemiptera: Miridae) control measures available to commercial producers of any flower crop in Florida.

\begin{tabular}{|c|c|c|c|}
\hline Common Name & Trade Name/Formulation & $\begin{array}{l}\text { Production } \\
\text { Site }^{1}\end{array}$ & Notes from Label ${ }^{2}$ \\
\hline $\begin{array}{l}\text { Pyrethrins, Piperonyl } \\
\text { Butoxide \& Silicon } \\
\text { Dioxide }\end{array}$ & $\begin{array}{l}\text { Diatect Multipurpose } \\
\text { Insecticide II }\end{array}$ & $\mathrm{F}$ & \\
\hline $\begin{array}{l}\text { Pyrethrins, Rotenone \& } \\
\text { other Cube Resins }\end{array}$ & Pyrellin E.C. & $F, G$ & Apply in intervals of 7 days or less. \\
\hline \multirow[t]{2}{*}{$\begin{array}{l}\text { Refined Petroleum } \\
\text { Distillate }\end{array}$} & Saf-T-Side Spray Oil & $\mathrm{F}$ & $\begin{array}{l}\text { This insecticide's mode of action is through } \\
\text { suffocation of eggs, larvae and nymphs of } \\
\text { insects and adults of soft bodied insects. This } \\
\text { mode of action necessitates total spray } \\
\text { coverage. Bedding plants; do not use in } \\
\text { combination with or immediately before or } \\
\text { after spraying with fungicides such as captan, } \\
\text { oxythioquinox or any product containing } \\
\text { sulfur. Also do not use with carbaryl (Sevin) } \\
\text { or dimethoate. Do not use with any product } \\
\text { whose label recommends the use of no oils. } \\
\text { Do not use in combination with NPK foliar } \\
\text { fertilizer applications. }\end{array}$ \\
\hline & Ultra-Fine Oil & $\mathrm{F}$ & $\begin{array}{l}\text { Honeylocust plant bugs; caution: Spray no } \\
\text { more than } 4 \text { consecutive sprays. The } \\
\text { frequency of consecutive sprays should not } \\
\text { exceed once every } 2 \text { weeks. Early morning } \\
\text { applications are recommended. Do not tank } \\
\text { mix with insecticide or miticide whose label } \\
\text { indicates that it should not be used with oil. } \\
\text { Do not use with captan, chlorothalonil, } \\
\text { dimethoate, methiocarb, oxythioquinox, } \\
\text { propargite, or any product containing sulfur. } \\
\text { This list is not exhaustive; therefore for } \\
\text { products not included in this list consult label } \\
\text { for compatibility information. }\end{array}$ \\
\hline Tau-Fluvalinate & Mavrik Aquaflow & $F, G$ & $\begin{array}{l}\text { Repeat applications not more than four times } \\
\text { per month at 5-7 day intervals unless } \\
\text { otherwise noted in use directions. }\end{array}$ \\
\hline Thiamethoxam & Flagship 25WG & $F, G$ & $\begin{array}{l}\text { Shade house, container \& nursery grown }(\mathrm{F}) \text {; } \\
\text { do not exceed } 8 \mathrm{oz} \text {. per acre per crop or year, } \\
\text { which ever is shorter. }\end{array}$ \\
\hline \multicolumn{4}{|c|}{$\begin{array}{l}\text { Footnotes: } \\
1 \text { "F" indicates field production. "G" indicates greenhouse production. } \\
2 \text { Notes are taken from product labels and restrict use to the condition indicated (suppression, beet armyworm, exposed } \\
\text { thrips, etc.), limit numbers or patterns of applications, provide phytotoxicity precautions, etc. }\end{array}$} \\
\hline
\end{tabular}


Table 18. Shore fly (Diptera: Ephydridae) control measures available to commercial producers of any flower crop in Florida.

\begin{tabular}{|c|c|c|c|}
\hline Common Name & Trade Name/Formulation & $\begin{array}{l}\text { Production } \\
\text { Site }^{1}\end{array}$ & Notes from Label $^{2}$ \\
\hline Azadirachtin & $\begin{array}{l}\text { Azatin XL } \\
\text { Ornazin 3\% EC }\end{array}$ & $F, G$ & \\
\hline $\begin{array}{l}\text { Beauveria bassiana } \\
\text { ATCC } 74040\end{array}$ & Naturalis L & $F, G$ & $\begin{array}{l}\text { Do not tank mix with fungicides. Wait a } \\
\text { minimum of } 48 \text { hours after application before } \\
\text { applying fungicides. }\end{array}$ \\
\hline Chlorpyrifos & Duragard ME & $F, G$ & $\begin{array}{l}\text { Nursery (F); bedding plants, potted and cut } \\
\text { flowers. Direct spray to some open blooms } \\
\text { may cause petal drop. Do not spray on } \\
\text { kalanchoes. }\end{array}$ \\
\hline Chlorpyrifos \& Cyfluthrin & Duraplex TR & $G$ & \\
\hline Cyfluthrin & Decathlon $20 \mathrm{WP}$ & $F, G$ & Flies \\
\hline Cyromazine & Citation & $\mathrm{G}$ & Will not control adult stages. \\
\hline Deltamethrin & Deltagard GC 5 SC & $\mathrm{F}$ & Flies; nursery \\
\hline \multirow[t]{2}{*}{ Diazinon } & $\begin{array}{l}\text { Diazinon 50W (EPA\# } \\
\text { 100-460-34704) } \\
\text { Diazinon AG } 500\end{array}$ & $\mathrm{~F}$ & $\begin{array}{l}\text { Flies (larvae); precaution: Do not use on } \\
\text { ferns, poinsettias, hibiscus, pilea, and } \\
\text { gardenia since injury to the plants may occur. }\end{array}$ \\
\hline & KnoxOut NL & $\mathrm{F}$ & Larvae; nursery \\
\hline Diflubenzuron & Adept & $F, G$ & $\begin{array}{l}\text { Shade house }(F) \text {; do not make more than four } \\
\text { applications per crop. Do not apply to } \\
\text { poinsettias, hibiscus, and Rieger begonia. Do } \\
\text { not reuse potting media, which has been } \\
\text { treated with Adept. Do not apply to plants } \\
\text { grown on capillary water mats. }\end{array}$ \\
\hline Fenoxycarb & Precision & $F, G$ & $\begin{array}{l}\text { Scatella spp.; Container grown }(F) \text { or shade } \\
\text { house grown }(F) \text {; will not control the adult } \\
\text { stages of insect pests. Do not exceed a } \\
\text { maximum of } 30 \text { applications per year for } \\
\text { greenhouse use. Applications should not be } \\
\text { made to poinsettia after bract formation. }\end{array}$ \\
\hline $\begin{array}{l}\text { Potassium Salts of Fatty } \\
\text { Acids }\end{array}$ & M-Pede & $F, G$ & $\begin{array}{l}\text { Tank mixes with chlorothalonil should be } \\
\text { avoided. Potential for injury increases on } \\
\text { plants stressed by heat, humidity, drought, or } \\
\text { insect, mite or disease pressure. Do not apply } \\
\text { to very sensitive plants such as: Cherimoya, } \\
\text { bleeding heart, or sweetpeas. The following } \\
\text { may be sensitive: Crown of thorns and other } \\
\text { euphorbia, fuchsia, gardenia, impatiens, } \\
\text { Asiatic and oriental lily varieties, lantana, } \\
\text { ornamental ivy, poinsettia, redbud, } \\
\text { schefflera, zebra plant and some succulents. } \\
\text { Flowers of the following may be injured when } \\
\text { sprayed: African violet, ageratum, azalea, } \\
\text { begonia, camellia, chrysanthemum, dahlia, } \\
\text { geranium, gloxinia, impatiens, lily, marigold, } \\
\text { orchid, pansy, petunia, poinsettia bracts, } \\
\text { rose, salvia, snapdragon, vinca, and zinnia. }\end{array}$ \\
\hline
\end{tabular}


Table 18. Shore fly (Diptera: Ephydridae) control measures available to commercial producers of any flower crop in Florida.

\begin{tabular}{|c|c|c|c|}
\hline Common Name & Trade Name/Formulation & $\begin{array}{l}\text { Production } \\
\text { Site }^{1}\end{array}$ & Notes from Label $^{2}$ \\
\hline \multirow{2}{*}{$\begin{array}{l}\text { Pyrethrins \& } \\
\text { Piperonyl Butoxide }\end{array}$} & 1100 Pyrethrum TR & $G$ & Flies \\
\hline & Pyrenone Crop Spray & $F, G$ & Flies \\
\hline $\begin{array}{l}\text { Pyrethrins, } \\
\text { Piperonyl Butoxide \& } \\
\text { Silicon Dioxide }\end{array}$ & $\begin{array}{l}\text { Diatect Multipurpose } \\
\text { Insecticide II }\end{array}$ & $\mathrm{F}$ & Flies \\
\hline Pyriproxyfen & Distance IGR & $F, G$ & $\begin{array}{l}\text { For foliar spray application, apply Distance no } \\
\text { more than two times per cropping cycle or no } \\
\text { more than two times per } 6 \text { months. For } \\
\text { sprench application, if a second application is } \\
\text { needed, allow a minimum of } 21 \text { days between } \\
\text { applications. For drench application: Do not } \\
\text { drench plants more than one time per crop } \\
\text { cycle. Phytotoxicity has been observed on } \\
\text { salvia (Salvia spp.), ghost plant } \\
\text { (Graptopetalum paraguayense), schefflera } \\
\text { (Schefflera spp.), gardenia (Gardenia spp.), } \\
\text { and coral bells (Heuchera sanguinea). It is } \\
\text { therefore recommended that Distance not be } \\
\text { used on these plants. Do not apply to } \\
\text { poinsettia after bract formation. }\end{array}$ \\
\hline $\begin{array}{l}\text { Steinernema } \\
\text { carpocapsae }\end{array}$ & Millenium & $F, G$ & Nursery $(F)$ \\
\hline \multicolumn{4}{|c|}{$\begin{array}{l}\text { Footnotes: } \\
1 \text { "F" indicates field production. "G" indicates greenhouse production. } \\
2 \text { Notes are taken from product labels and restrict use to the condition indicated (suppression, beet armyworm, exposed } \\
\text { thrips, etc.), limit numbers or patterns of applications, provide phytotoxicity precautions, etc. }\end{array}$} \\
\hline
\end{tabular}

Table 19. Slugs \& snails (Mollusca: Gastropoda: Pulmonata: Limacidae)) control measures available to commercial producers of any flower crop in Florida.

\begin{tabular}{|c|c|c|c|}
\hline Common Name & Trade Name/Formulation & $\begin{array}{l}\text { Production } \\
\text { Site }^{1}\end{array}$ & Notes from Label ${ }^{2}$ \\
\hline Iron phosphate & Monterey Sluggo-Ag & $F, G$ & $\begin{array}{l}\text { Deroceras reticulatum (field slug); Deroceras } \\
\text { laeve (smooth slug); Arion subfuscus (dusky } \\
\text { slug); Arion circumscriptus (gray garden } \\
\text { slug); Arion hortensis (black field slug); Arion } \\
\text { rufus (large red slug); Arios ater (large black } \\
\text { slug); Limax flavus (spotted garden slug); } \\
\text { Limax tenellus (slender slug); } \\
\text { Ariolimax columbianus (banana slug); Helix } \\
\text { spp.; } \\
\text { Helicella spp.; Cepaea spp. }\end{array}$ \\
\hline
\end{tabular}


Table 19. Slugs \& snails (Mollusca: Gastropoda: Pulmonata: Limacidae)) control measures available to commercial producers of any flower crop in Florida.

\begin{tabular}{|c|c|c|c|}
\hline Common Name & Trade Name/Formulation & $\begin{array}{l}\text { Production } \\
\text { Site }^{1}\end{array}$ & Notes from Label $^{2}$ \\
\hline \multirow[t]{4}{*}{ Metaldehyde } & $\begin{array}{l}\text { Deadline Bullets } \\
\text { Deadline M-Ps }\end{array}$ & $F, G$ & $\begin{array}{l}\text { Nursery }(F) \text {; for best results apply in } \\
\text { evening. }\end{array}$ \\
\hline & $\begin{array}{l}\text { Durham Granules } 3.5 \\
\text { Durham Granules } 7.5 \\
\text { Trails End } 3.5 \\
\text { Trails End LG }\end{array}$ & $G$ & $\begin{array}{l}\text { Do not apply directly on to plants. Use only } \\
\text { as a baiting treatment ... [Product] should } \\
\text { never be applied to dry soil. Applications } \\
\text { should be made following irrigation for best } \\
\text { results. Apply any time of the day but evening } \\
\text { applications are preferred. Do not re-water for } \\
48 \text { hours after application. Some slight tip } \\
\text { burning has been noticed on extremely tender } \\
\text { orchids. For heavily infested greenhouses a } \\
\text { second application is recommended } 7-10 \\
\text { days following the first application. }\end{array}$ \\
\hline & Prozap Snail \& Slug AG & $F, G$ & $\begin{array}{l}\text { Use only as a baiting treatment ... [Product] } \\
\text { should never be applied to dry soil. } \\
\text { Applications should be made following } \\
\text { irrigation for best results. Apply any time of } \\
\text { the day but evening applications are } \\
\text { preferred. Do not re-water for } 48 \text { hours after } \\
\text { application. Some slight tip burning has been } \\
\text { noticed on extremely tender orchids. For } \\
\text { heavily infested greenhouses a second } \\
\text { application is recommended } 7-10 \text { days } \\
\text { following the first application. }\end{array}$ \\
\hline & $\begin{array}{l}\text { Slug-Fest All Weather } \\
\text { Formula }\end{array}$ & $F, G$ & $\begin{array}{l}\text { Do not apply directly to plants unless prior } \\
\text { experience has shown the plant to be } \\
\text { tolerant, however some slight tip burning has } \\
\text { been noticed on extremely tender orchids. } \\
\text { Thoroughly water areas to be treated before } \\
\text { application. For best results apply in the } \\
\text { evening. }\end{array}$ \\
\hline Methiocarb & $\begin{array}{l}\text { Mesurol 75-W } \\
\text { Mesurol Pro }\end{array}$ & $F, G$ & Nursery (F); do not apply with oil. \\
\hline \multicolumn{4}{|c|}{$\begin{array}{l}\text { Footnotes: } \\
{ }^{1} \text { "F" indicates field production. "G" indicates greenhouse production. } \\
{ }^{2} \text { Notes are taken from product labels and restrict use to the condition indicated (suppression, beet armyworm, exposed } \\
\text { thrips, etc.), limit numbers or patterns of applications, provide phytotoxicity precautions, etc. }\end{array}$} \\
\hline
\end{tabular}


Table 20. Thrips (Thysanoptera: Thripidae) control measures available to commercial producers of any flower crop in Florida.

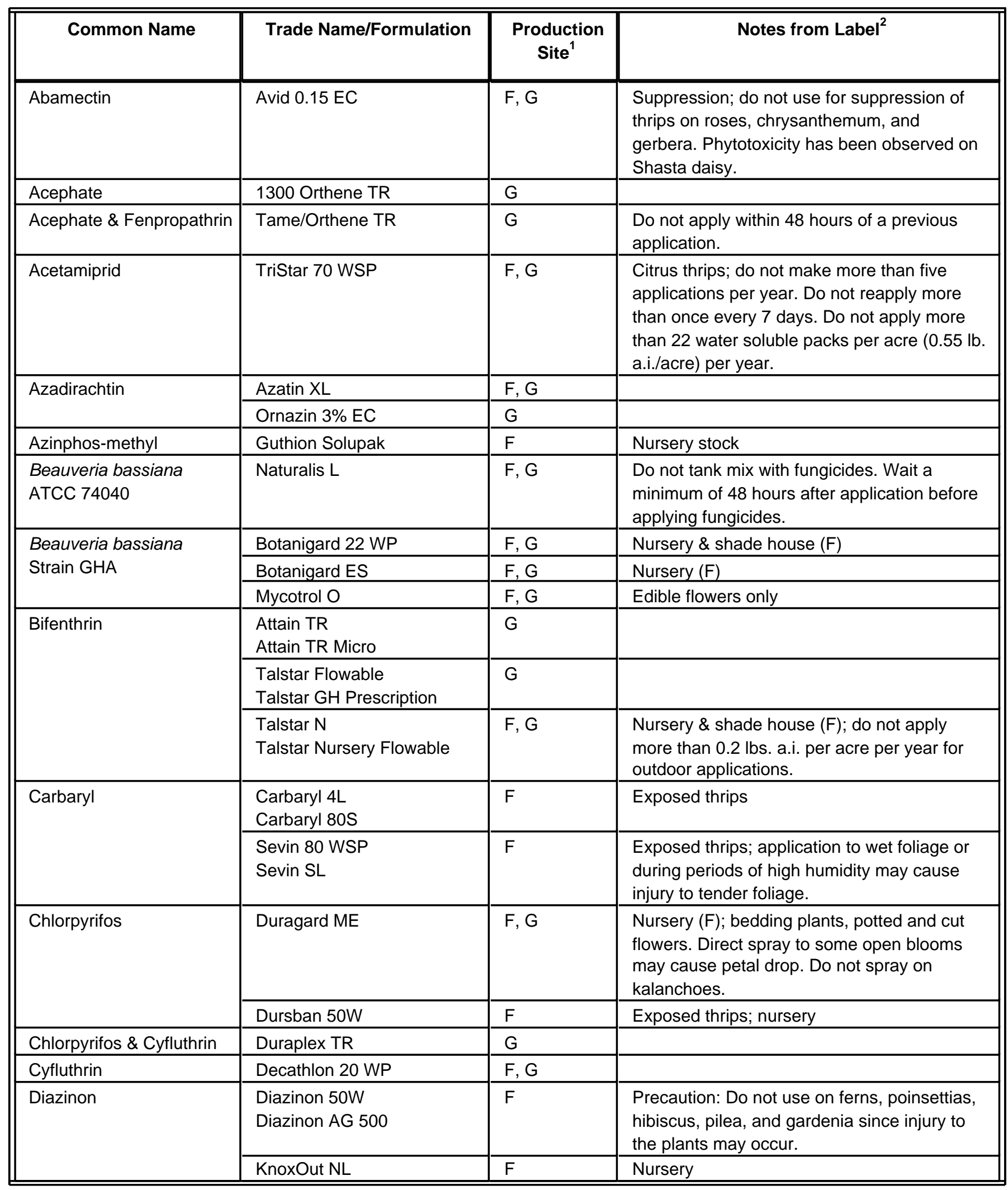


Table 20. Thrips (Thysanoptera: Thripidae) control measures available to commercial producers of any flower crop in Florida.

\begin{tabular}{|c|c|c|c|}
\hline Common Name & Trade Name/Formulation & $\begin{array}{l}\text { Production } \\
\text { Site }^{1}\end{array}$ & Notes from Label $^{2}$ \\
\hline \multirow[t]{2}{*}{ Fenoxycarb } & Precision & $F, G$ & $\begin{array}{l}\text { Container grown }(F) \text { or shade house grown } \\
(F) \text {; will not control the adult stages of insect } \\
\text { pests. Do not exceed a maximum of } 30 \\
\text { applications per year for greenhouse use. } \\
\text { Applications should not be made to poinsettia } \\
\text { after bract formation. }\end{array}$ \\
\hline & Preclude TR & $\mathrm{G}$ & \\
\hline Fenpropathrin & Tame 2.4EC & $F, G$ & $\begin{array}{l}\text { Nursery \& shade house }(F) \text {; do not use less } \\
\text { than a } 7 \text { day re-treatment interval. }\end{array}$ \\
\hline \multirow[t]{3}{*}{ Imidacloprid } & Marathon $1 \%$ Granular & $F, G$ & $\begin{array}{l}\text { Suppression; nursery }(\mathrm{F}) \text {; for outdoor } \\
\text { ornamentals grown in beds, applications can } \\
\text { not exceed a total of } 40 \mathrm{lbs} \text {. per acre per year. } \\
\text { On plants with a production cycle of less than } \\
1 \text { year, application is not to exceed a } \\
\text { frequency of more than once each } 16 \text { weeks } \\
\text { for a particular plant. On stock plants and } \\
\text { woody crops with a production cycle of } \\
\text { greater than } 1 \text { year, application may not } \\
\text { exceed once a year. }\end{array}$ \\
\hline & Marathon $60 \mathrm{WP}$ & $F, G$ & $\begin{array}{l}\text { Suppression; nursery }(\mathrm{F}) \text {; this product is to be } \\
\text { applied as a soil treatment only. For outdoor } \\
\text { ornamentals grown in beds applications can } \\
\text { not exceed a total of } 10.7 \mathrm{oz} \text {. ( } 0.4 \mathrm{lbs} \text {. active } \\
\text { ingredient) per acre per year. On plants with a } \\
\text { production cycle of less than } 1 \text { year, } \\
\text { application is not to exceed a frequency of } \\
\text { more than once each } 16 \text { weeks for a } \\
\text { particular plant. On stock plants and woody } \\
\text { crops with a production cycle of greater than } \\
1 \text { year, application may not exceed once a } \\
\text { year. }\end{array}$ \\
\hline & Marathon II & $F, G$ & $\begin{array}{l}\text { Suppression; for outdoor ornamentals do not } \\
\text { apply more than } 1.6 \mathrm{pt} \text {. ( } 0.4 \mathrm{lbs} \text {. a.i.) per acre } \\
\text { per year. Thrips are suppressed on foliage } \\
\text { only, not in buds or flowers. Do not apply thru } \\
\text { any type of irrigation system. }\end{array}$ \\
\hline Lambda-cyhalothrin & Scimitar GC & $F, G$ & $\begin{array}{l}\text { Nursery \& shade house (F); do not apply } \\
\text { more than } 0.36 \text { lbs. a.i. ( } 52.4 \text { fl. oz. of } \\
\text { concentrate) per acre per year. }\end{array}$ \\
\hline Malathion & $\begin{array}{l}\text { Atrapa } 5 \mathrm{E} \\
\text { Gowan Malathion } 8 \mathrm{~F} \\
\text { Malathion } 5 \mathrm{EC}\end{array}$ & $\mathrm{F}$ & \\
\hline Methiocarb & Mesurol 75-W & $F, G$ & $\begin{array}{l}\text { Western flower thrips; nursery (F); do not } \\
\text { apply with oil. }\end{array}$ \\
\hline
\end{tabular}


Table 20. Thrips (Thysanoptera: Thripidae) control measures available to commercial producers of any flower crop in Florida.

\begin{tabular}{|c|c|c|c|}
\hline Common Name & Trade Name/Formulation & $\begin{array}{l}\text { Production } \\
\text { Site }^{1}\end{array}$ & Notes from Label $^{2}$ \\
\hline Novaluron & Pedestal & $F, G$ & $\begin{array}{l}\text { Container grown plants; nursery \& shade } \\
\text { house (F); do not apply more than once every } \\
30 \text { days. Do not make more than two } \\
\text { applications per crop year. Do not apply more } \\
\text { than } 52 \text { fl. oz. per acre per year per crop. Do } \\
\text { not apply to poinsettias. Resistance } \\
\text { management: Do not apply in successive } \\
\text { applications, use only one application before } \\
\text { rotation with other classes of insecticides with } \\
\text { different modes of action. Use at least two } \\
\text { alternative products between applications of } \\
\text { Pedestal. Do not use more than once within } \\
\text { each generation cycle. }\end{array}$ \\
\hline \multirow[t]{5}{*}{ Permethrin } & Ambush 25W & $\mathrm{F}$ & $\begin{array}{l}\text { Citrus thrips; field grown nursery stock; do } \\
\text { not apply more than } 2.0 \text { lbs. a.i. per acre per } \\
\text { season. Marginal leaf burn may occur on: } \\
\text { Salvia, dieffenbachia. Application to blooming } \\
\text { plants may cause browning of petals. }\end{array}$ \\
\hline & $\begin{array}{l}\text { Ambush } \\
\text { Pounce } 25 \text { WP } \\
\text { Pounce } 3.2 \text { EC } \\
\text { Pounce WSB } \\
\end{array}$ & $\mathrm{F}$ & Citrus thrips; field grown nursery stock. \\
\hline & Astro & $G$ & $\begin{array}{l}\text { Citrus thrips; do not apply more than } 2.0 \mathrm{lb} \text {. } \\
\text { a.i. per acre per year. }\end{array}$ \\
\hline & $\begin{array}{l}\text { Clean Crop Permethrin } 3.2 \\
\text { EC }\end{array}$ & $\mathrm{F}$ & $\begin{array}{l}\text { Citrus thrips; field grown nursery stock; } \\
\text { marginal leaf burn may occur on: Salvia, } \\
\text { dieffenbachia. Application to blooming plants } \\
\text { may cause browning of petals. Avoid } \\
\text { spraying chrysanthemum blooms. }\end{array}$ \\
\hline & Perm-Up 3.2EC & $\mathrm{F}$ & $\begin{array}{l}\text { Citrus thrips; field grown nursery stock; } \\
\text { marginal leaf burn may occur on: Salvia, } \\
\text { dieffenbachia. }\end{array}$ \\
\hline
\end{tabular}


Table 20. Thrips (Thysanoptera: Thripidae) control measures available to commercial producers of any flower crop in Florida.

\begin{tabular}{|c|c|c|c|}
\hline Common Name & Trade Name/Formulation & $\begin{array}{l}\text { Production } \\
\text { Site }^{1}\end{array}$ & Notes from Label ${ }^{2}$ \\
\hline \multirow[t]{2}{*}{$\begin{array}{l}\text { Potassium Salts of Fatty } \\
\text { Acids }\end{array}$} & Insecticidal Soap 49.52 CF & $F, G$ & $\begin{array}{l}\text { Caution: Do not use on new transplants, } \\
\text { newly rooted cuttings, plants stressed by } \\
\text { drought, or when tender new foliage is } \\
\text { present. Avoid application when leaf } \\
\text { temperature exceeds } 85^{\circ} \mathrm{F} \text {. Note: Do not } \\
\text { use on crown of thorns and test on other } \\
\text { euphorbias for phytotoxicity. Do not use on } \\
\text { bleeding heart, lantana, lilies or sweetpeas. } \\
\text { Some varieties of azaleas, begonias, } \\
\text { camellias, fuschias, gardenias, orchids, and } \\
\text { impatiens have shown sensitivity. Do not } \\
\text { apply to poinsettias after bract coloration } \\
\text { begins. Test on chrysanthemums for varietal } \\
\text { sensitivity and do not apply to open blooms. } \\
\text { Do not apply to ornamentals stressed by } \\
\text { drought. Test on eugenia and euonymus for } \\
\text { varietal and drought sensitivity. }\end{array}$ \\
\hline & M-Pede & $F, G$ & $\begin{array}{l}\text { Exposed thrips; tank mixes with chlorothalonil } \\
\text { should be avoided. Potential for injury } \\
\text { increases on plants stressed by heat, } \\
\text { humidity, drought, or insect, mite or disease } \\
\text { pressure. Do not apply to very sensitive } \\
\text { plants such as: Cherimoya, bleeding heart, or } \\
\text { sweetpeas. The following may be sensitive: } \\
\text { Crown of thorns and other euphorbia, fuchsia, } \\
\text { gardenia, impatiens, Asiatic and oriental lily } \\
\text { varieties, lantana, ornamental ivy, poinsettia, } \\
\text { redbud, schefflera, zebra plant and some } \\
\text { succulents. Flowers of the following may be } \\
\text { injured when sprayed: African violet, } \\
\text { ageratum, azalea, begonia, camellia, } \\
\text { chrysanthemum, dahlia, geranium, gloxinia, } \\
\text { impatiens, lily, marigold, orchid, pansy, } \\
\text { petunia, poinsettia bracts, rose, salvia, } \\
\text { snapdragon, vinca, and zinnia. }\end{array}$ \\
\hline \multirow{2}{*}{$\begin{array}{l}\text { Pyrethrins \& } \\
\text { Piperonyl Butoxide }\end{array}$} & 1100 Pyrethrum TR & $\mathrm{G}$ & \\
\hline & $\begin{array}{l}\text { Pyrenone Crop Spray } \\
\text { Pyreth-It }\end{array}$ & $\mathrm{F}, \mathrm{G}$ & \\
\hline $\begin{array}{l}\text { Pyrethrins, } \\
\text { Piperonyl Butoxide \& } \\
\text { Silicon Dioxide } \\
\end{array}$ & $\begin{array}{l}\text { Diatect Multipurpose } \\
\text { Insecticide II }\end{array}$ & $\mathrm{F}$ & \\
\hline $\begin{array}{l}\text { Pyrethrins, Rotenone \& } \\
\text { other Cube Resins }\end{array}$ & Pyrellin E.C. & $F, G$ & Apply in intervals of 7 days or less. \\
\hline
\end{tabular}


Table 20. Thrips (Thysanoptera: Thripidae) control measures available to commercial producers of any flower crop in Florida.

\begin{tabular}{|c|c|c|c|}
\hline Common Name & Trade Name/Formulation & $\begin{array}{l}\text { Production } \\
\text { Site }^{1}\end{array}$ & Notes from Label $^{2}$ \\
\hline S-Kinoprene & Enstar II & $\mathrm{G}$ & $\begin{array}{l}\text { Application should be made to poinsettia } \\
\text { before bract formation. Foliar damage on } \\
\text { some sensitive varieties can result. Some } \\
\text { varieties of roses, such as Yellow Blooded } \\
\text { roses, show delayed damage. Slight to } \\
\text { moderate injury has occurred on some } \\
\text { blooms under certain conditions, suggest } \\
\text { application be made in pre-bloom stage. }\end{array}$ \\
\hline Spinosad & Conserve SC & $F, G$ & $\begin{array}{l}\text { Exposed thrips; when used in greenhouse or } \\
\text { areas of commercial production of } \\
\text { herbaceous (non-woody) ornamentals in } \\
\text { nurseries (including plant propagation beds): } \\
\text { Do not apply more than } 10 \text { times in a } \\
12 \text {-month period per crop regardless of the } \\
\text { pest being treated. Because generations of a } \\
\text { specific pest may overlap, rotate control } \\
\text { products and never apply more than three } \\
\text { consecutive applications of Conserve SC or } \\
\text { products containing the same a.i. or products } \\
\text { with the same mode of action. Except for } \\
\text { greenhouses and structures that can be } \\
\text { altered to be closed or open, do not reapply } \\
\text { within less than } 7 \text { days. }\end{array}$ \\
\hline Tau-Fluvalinate & Mavrik Aquaflow & $F, G$ & $\begin{array}{l}\text { Repeat applications not more than four times } \\
\text { per month at } 5-7 \text { day intervals unless } \\
\text { otherwise noted in use directions. }\end{array}$ \\
\hline \multicolumn{4}{|c|}{$\begin{array}{l}\text { Footnotes: } \\
1 \text { "F" indicates field production. "G" indicates greenhouse production. } \\
{ }^{2} \text { Notes are taken from product labels and restrict use to the condition indicated (suppression, beet armyworm, exposed } \\
\text { thrips, etc.), limit numbers or patterns of applications, provide phytotoxicity precautions, etc. }\end{array}$} \\
\hline
\end{tabular}

Table 21. Whitefly (Homoptera: Aleyrodidae) control measures available to commercial producers of any flower crop in Florida.

\begin{tabular}{||l|l|l|l||}
\hline \hline \multicolumn{1}{||c|}{ Common Name } & \multicolumn{1}{|c|}{ Trade Name/Formulation } & $\begin{array}{c}\text { Production } \\
\text { Site }^{1}\end{array}$ & \multicolumn{1}{|c||}{ Notes from Label $^{2}$} \\
\hline Abamectin & Avid 0.15 EC & F, G & $\begin{array}{l}\text { Suppression; do not use for suppression of } \\
\text { whiteflies on roses, chrysanthemum, and } \\
\text { gerbera. Phytotoxicity has been observed } \\
\text { on Shasta daisy. }\end{array}$ \\
\hline Acephate $^{3}$ & 1300 Orthene TR & G & $\begin{array}{l}\text { Do not apply within 48 hours of a previous } \\
\text { application. }\end{array}$ \\
\hline $\begin{array}{l}\text { Acephate \& } \\
\text { Fenpropathrin }\end{array}$ & Tame/Orthene TR & G & \\
\hline \hline
\end{tabular}


Table 21. Whitefly (Homoptera: Aleyrodidae) control measures available to commercial producers of any flower crop in Florida.

\begin{tabular}{|c|c|c|c|}
\hline Common Name & Trade Name/Formulation & $\begin{array}{l}\text { Production } \\
\text { Site }^{1}\end{array}$ & Notes from Label ${ }^{2}$ \\
\hline Acetamiprid & TriStar 70 WSP & $F, G$ & $\begin{array}{l}\text { Do not make more than five applications } \\
\text { per year. Do not reapply more than once } \\
\text { every } 7 \text { days. Do not apply more than } 22 \\
\text { water soluble packs per acre }(0.55 \mathrm{lb} \text {. } \\
\text { a.i./acre) per year. }\end{array}$ \\
\hline Azadirachtin & $\begin{array}{l}\text { Azatin XL } \\
\text { Ornazin 3\% EC }\end{array}$ & $F, G$ & \\
\hline $\begin{array}{l}\text { Beauveria bassiana } \\
\text { ATCC } 74040\end{array}$ & Naturalis L & $F, G$ & $\begin{array}{l}\text { Do not tank mix with fungicides. Wait a } \\
\text { minimum of } 48 \text { hours after application } \\
\text { before applying fungicides. }\end{array}$ \\
\hline \multirow{2}{*}{$\begin{array}{l}\text { Beauveria bassiana } \\
\text { Strain GHA }\end{array}$} & Botanigard 22 WP & $F, G$ & Nursery \& shade house $(F)$ \\
\hline & Botanigard ES & $F, G$ & Nursery (F) \\
\hline \multirow[t]{3}{*}{ Bifenthrin } & $\begin{array}{l}\text { Attain TR } \\
\text { Attain TR Micro } \\
\end{array}$ & $\mathrm{G}$ & \\
\hline & $\begin{array}{l}\text { Talstar Flowable } \\
\text { Talstar GH Prescription } \\
\end{array}$ & $\mathrm{G}$ & \\
\hline & $\begin{array}{l}\text { Talstar N } \\
\text { Talstar Nursery Flowable }\end{array}$ & $F, G$ & $\begin{array}{l}\text { Nursery \& shade house }(\mathrm{F}) \text {; do not apply } \\
\text { more than } 0.2 \text { lbs. a.i. per acre per year for } \\
\text { outdoor applications. }\end{array}$ \\
\hline Buprofezin & $\begin{array}{l}\text { Talus Insect Growth } \\
\text { Regulator }\end{array}$ & $F, G$ & $\begin{array}{l}\text { Silverleaf, sweet potato and greenhouse } \\
\text { whitefly; to preserve usefulness of Talus, do } \\
\text { not make more than two consecutive } \\
\text { applications. If another insect growth } \\
\text { regulator with the mode of action as a chitin } \\
\text { synthesis inhibitor (ie. cyromazine, } \\
\text { diflubenzuron, novaluron) has been used in } \\
\text { the greenhouse within } 28 \text { days, do not } \\
\text { apply Talus as the first whitefly application. } \\
\text { Prior to subsequent applications, use an } \\
\text { alternative chemistry with a different mode } \\
\text { of action. }\end{array}$ \\
\hline \multirow[t]{2}{*}{ Chlorpyrifos } & Chlorpyrifos Pro 2 & $\mathrm{~F}$ & Nursery stock in nurseries \\
\hline & Dursban 50W & $\mathrm{F}$ & Nursery \\
\hline Chlorpyrifos \& Cyfluthrin & Duraplex TR & $G$ & \\
\hline $\begin{array}{l}\text { Clarified Hydrophobic } \\
\text { Extract of Neem Oil }\end{array}$ & Triact 70 & $F, G$ & $\begin{array}{l}\text { Test before applying to carnation and the } \\
\text { flowers of: Impatiens, fuchsia, hibiscus, and } \\
\text { some roses. Most effective when applied } \\
\text { early to mid-morning or late afternoon. }\end{array}$ \\
\hline Cyfluthrin & Decathlon $20 \mathrm{WP}$ & $\mathrm{F}, \mathrm{G}$ & \\
\hline \multirow[t]{2}{*}{ Diazinon } & $\begin{array}{l}\text { Diazinon 50W } \\
\text { Diazinon AG } 500\end{array}$ & $\mathrm{~F}$ & $\begin{array}{l}\text { Precaution: Do not use on ferns, } \\
\text { poinsettias, hibiscus, pilea, and gardenia } \\
\text { since injury to the plants may occur. }\end{array}$ \\
\hline & KnoxOut NL & $\mathrm{F}$ & Suppression; nursery \\
\hline
\end{tabular}


Pesticide Options for Important Insect, Mite and Mollusk Pests of Commercial Flowers in....

Table 21. Whitefly (Homoptera: Aleyrodidae) control measures available to commercial producers of any flower crop in Florida.

\begin{tabular}{|c|c|c|c|}
\hline Common Name & Trade Name/Formulation & $\begin{array}{l}\text { Production } \\
\text { Site }^{1}\end{array}$ & Notes from Label ${ }^{2}$ \\
\hline \multirow[t]{3}{*}{ Diflubenzuron } & Adept & $F, G$ & $\begin{array}{l}\text { Suppression; shade house (F); do not make } \\
\text { more than four applications per crop. Do } \\
\text { not apply to poinsettias, hibiscus, and } \\
\text { Rieger begonia. Do not reuse potting } \\
\text { media, which has been treated with Adept. } \\
\text { Do not apply to plants grown on capillary } \\
\text { water mats. }\end{array}$ \\
\hline & Dimilin 25W & $\mathrm{F}, \mathrm{G}$ & Suppression; shade house (F) \\
\hline & Dimilin SC & $\mathrm{F}, \mathrm{G}$ & Suppression; shade house (F) \\
\hline Endosulfan & $\begin{array}{l}\text { Endosulfan } 3 \text { EC } \\
\text { Endosulfan } 50 \text { WP } \\
\text { Thionex (Endosulfan) } 3 \text { EC } \\
\text { Thionex (Endosulfan) } 50 \text { WP } \\
\end{array}$ & $F, G$ & $\begin{array}{l}\text { Do not exceed } 3.0 \text { lbs. a.i. per acre per } \\
\text { year. On chrysanthemums, best results will } \\
\text { be obtained if applied before plants flower. }\end{array}$ \\
\hline \multirow[t]{2}{*}{ Fenoxycarb } & Precision & $F, G$ & $\begin{array}{l}\text { Silverleaf (alias sweetpotato) whitefly, } \\
\text { greenhouse whitefly; container grown (F) or } \\
\text { shade house grown (F); will not control the } \\
\text { adult stages of insect pests. Do not exceed } \\
\text { a maximum of } 30 \text { applications per year for } \\
\text { greenhouse use. Applications should not be } \\
\text { made to poinsettia after bract formation. }\end{array}$ \\
\hline & Preclude TR & $\mathrm{G}$ & \\
\hline Fenpropathrin & Tame 2.4EC & $F, G$ & $\begin{array}{l}\text { Silverleaf/sweetpotato whitefly (includes all } \\
\text { instars and transitional stages [pupae]), } \\
\text { greenhouse whitefly; nursery \& shade } \\
\text { house }(F) \text {; do not use less than a } 7 \text { day } \\
\text { re-treatment interval. }\end{array}$ \\
\hline
\end{tabular}


Table 21. Whitefly (Homoptera: Aleyrodidae) control measures available to commercial producers of any flower crop in Florida.

\begin{tabular}{|c|c|c|c|}
\hline Common Name & Trade Name/Formulation & $\begin{array}{l}\text { Production } \\
\text { Site }^{1}\end{array}$ & Notes from Label $^{2}$ \\
\hline \multirow[t]{3}{*}{ Imidacloprid } & Marathon $1 \%$ Granular & $F, G$ & $\begin{array}{l}\text { Nursery }(F) \text {; for outdoor ornamentals grown } \\
\text { in beds, applications can not exceed a total } \\
\text { of } 40 \mathrm{lbs} \text {. per acre per year. On plants with a } \\
\text { production cycle of less than } 1 \text { year, } \\
\text { application is not to exceed a frequency of } \\
\text { more than once each } 16 \text { weeks for a } \\
\text { particular plant. On stock plants and woody } \\
\text { crops with a production cycle of greater } \\
\text { than } 1 \text { year, application may not exceed } \\
\text { once a year. }\end{array}$ \\
\hline & Marathon $60 \mathrm{WP}$ & $F, G$ & $\begin{array}{l}\text { Nursery }(\mathrm{F}) \text {; this product is to be applied as } \\
\text { a soil treatment only. For outdoor } \\
\text { ornamentals grown in beds applications } \\
\text { can not exceed a total of } 10.7 \mathrm{oz} \text {. ( } 0.4 \mathrm{lbs} \text {. } \\
\text { active ingredient) per acre per year. On } \\
\text { plants with a production cycle of less than } 1 \\
\text { year, application is not to exceed a } \\
\text { frequency of more than once each } 16 \\
\text { weeks for a particular plant. On stock plants } \\
\text { and woody crops with a production cycle of } \\
\text { greater than } 1 \text { year, application may not } \\
\text { exceed once a year. }\end{array}$ \\
\hline & Marathon II & $F, G$ & $\begin{array}{l}\text { For outdoor ornamentals do not apply more } \\
\text { than } 1.6 \text { pt. }(0.4 \text { lbs. a.i.) per acre per year. } \\
\text { Do not apply thru any type of irrigation } \\
\text { system. }\end{array}$ \\
\hline Malathion & $\begin{array}{l}\text { Atrapa 5E } \\
\text { Gowan Malathion } 8 \mathrm{~F} \\
\text { Malathion } 5 \mathrm{EC}\end{array}$ & $\mathrm{F}$ & \\
\hline Naled & Dibrom 8 Emulsive & $\bar{G}$ & $\begin{array}{l}\text { Adults; vapor treatment; spotting, bronzing } \\
\text { or localized burning can occur under certain } \\
\text { climatic conditions such as slow drying and } \\
\text { high humidity or stress caused by drought } \\
\text { or high temperature. White Butterfly and } \\
\text { Golden Rapture roses, Pink Champagne } \\
\text { chrysanthemums, green wandering jew, } \\
\text { poinsettias and Dutchman's pipe may be } \\
\text { injured by vapor. }\end{array}$ \\
\hline
\end{tabular}


Table 21. Whitefly (Homoptera: Aleyrodidae) control measures available to commercial producers of any flower crop in Florida.

\begin{tabular}{|c|c|c|c|}
\hline Common Name & Trade Name/Formulation & $\begin{array}{l}\text { Production } \\
\text { Site }^{1}\end{array}$ & Notes from Label $^{2}$ \\
\hline Novaluron & Pedestal & $F, G$ & $\begin{array}{l}\text { Container grown plants; nursery \& shade } \\
\text { house (F); do not apply more than once } \\
\text { every } 30 \text { days. Do not make more than two } \\
\text { applications per crop year. Do not apply } \\
\text { more than } 52 \text { fl. oz. per acre per year per } \\
\text { crop. Do not apply to poinsettias. } \\
\text { Resistance management: Do not apply in } \\
\text { successive applications, use only one } \\
\text { application before rotation with other } \\
\text { classes of insecticides with different modes } \\
\text { of action. Use at least two alternative } \\
\text { products between applications of Pedestal. } \\
\text { Do not use more than once within each } \\
\text { generation cycle. }\end{array}$ \\
\hline \multirow[t]{5}{*}{ Permethrin } & Ambush 25W & $\mathrm{F}$ & $\begin{array}{l}\text { Field grown nursery stock; do not apply } \\
\text { more than } 2.0 \text { lbs. a.i. per acre per season. } \\
\text { Marginal leaf burn may occur on: Salvia, } \\
\text { dieffenbachia. Application to blooming } \\
\text { plants may cause browning of petals. }\end{array}$ \\
\hline & $\begin{array}{l}\text { Ambush } \\
\text { Pounce 25 WP } \\
\text { Pounce } 3.2 \text { EC } \\
\text { Pounce WSB }\end{array}$ & $\mathrm{F}$ & Field grown nursery stock. \\
\hline & Astro & $G$ & $\begin{array}{l}\text { Do not apply more than } 2.0 \mathrm{lb} \text {. a.i. per acre } \\
\text { per year. }\end{array}$ \\
\hline & $\begin{array}{l}\text { Clean Crop Permethrin } 3.2 \\
\text { EC }\end{array}$ & $\mathrm{F}$ & $\begin{array}{l}\text { Field grown nursery stock; marginal leaf } \\
\text { burn may occur on: Salvia, dieffenbachia. } \\
\text { Application to blooming plants may cause } \\
\text { browning of petals. Avoid spraying } \\
\text { chrysanthemum blooms. }\end{array}$ \\
\hline & Perm-Up 3.2EC & $\mathrm{F}$ & $\begin{array}{l}\text { Field grown nursery stock; marginal leaf } \\
\text { burn may occur on: Salvia, dieffenbachia. }\end{array}$ \\
\hline
\end{tabular}


Table 21. Whitefly (Homoptera: Aleyrodidae) control measures available to commercial producers of any flower crop in Florida.

\begin{tabular}{|c|c|c|c|}
\hline Common Name & Trade Name/Formulation & $\begin{array}{l}\text { Production } \\
\text { Site }^{1}\end{array}$ & Notes from Label ${ }^{2}$ \\
\hline \multirow[t]{2}{*}{$\begin{array}{l}\text { Potassium Salts of Fatty } \\
\text { Acids }\end{array}$} & Insecticidal Soap 49.52 CF & $F, G$ & $\begin{array}{l}\text { Caution: Do not use on new transplants, } \\
\text { newly rooted cuttings, plants stressed by } \\
\text { drought, or when tender new foliage is } \\
\text { present. Avoid application when leaf } \\
\text { temperature exceeds } 85^{\circ} \text { F. Note: Do not } \\
\text { use on crown of thorns and test on other } \\
\text { euphorbias for phytotoxicity. Do not use on } \\
\text { bleeding heart, lantana, lilies or sweetpeas. } \\
\text { Some varieties of azaleas, begonias, } \\
\text { camellias, fuschias, gardenias, orchids, and } \\
\text { impatiens have shown sensitivity. Do not } \\
\text { apply to poinsettias after bract coloration } \\
\text { begins. Test on chrysanthemums for } \\
\text { varietal sensitivity and do not apply to open } \\
\text { blooms. Do not apply to ornamentals } \\
\text { stressed by drought. Test on eugenia and } \\
\text { euonymus for varietal and drought } \\
\text { sensitivity. }\end{array}$ \\
\hline & M-Pede & $F, G$ & $\begin{array}{l}\text { Tank mixes with chlorothalonil should be } \\
\text { avoided. Potential for injury increases on } \\
\text { plants stressed by heat, humidity, drought, } \\
\text { or insect, mite or disease pressure. Do not } \\
\text { apply to very sensitive plants such as: } \\
\text { Cherimoya, bleeding heart, or sweetpeas. } \\
\text { The following may be sensitive: Crown of } \\
\text { thorns and other euphorbia, fuchsia, } \\
\text { gardenia, impatiens, Asiatic and oriental lily } \\
\text { varieties, lantana, ornamental ivy, } \\
\text { poinsettia, redbud, schefflera, zebra plant } \\
\text { and some succulents. Flowers of the } \\
\text { following may be injured when sprayed: } \\
\text { African violet, ageratum, azalea, begonia, } \\
\text { camellia, chrysanthemum, dahlia, } \\
\text { geranium, gloxinia, impatiens, lily, marigold, } \\
\text { orchid, pansy, petunia, poinsettia bracts, } \\
\text { rose, salvia, snapdragon, vinca, and } \\
\text { zinnia. }\end{array}$ \\
\hline Pymetrozine & Endeavor & $F, G$ & $\begin{array}{l}\text { For outdoor use, do not apply more than } 48 \\
\text { oz. per acre per year. For indoor use, do not } \\
\text { apply more than } 100 \text { oz. per acre per year. }\end{array}$ \\
\hline \multirow{2}{*}{$\begin{array}{l}\text { Pyrethrins \& } \\
\text { Piperonyl Butoxide }\end{array}$} & 1100 Pyrethrum TR & $\mathrm{G}$ & \\
\hline & $\begin{array}{l}\text { Pyrenone Crop Spray } \\
\text { Pyreth-It }\end{array}$ & $F, G$ & \\
\hline $\begin{array}{l}\text { Pyrethrins, } \\
\text { Piperonyl Butoxide \& } \\
\text { Silicon Dioxide }\end{array}$ & $\begin{array}{l}\text { Diatect Multipurpose } \\
\text { Insecticide II }\end{array}$ & $\mathrm{F}$ & \\
\hline $\begin{array}{l}\text { Pyrethrins, Rotenone \& } \\
\text { other Cube Resins }\end{array}$ & Pyrellin E.C. & $F, G$ & Apply in intervals of 7 days or less. \\
\hline
\end{tabular}


Table 21. Whitefly (Homoptera: Aleyrodidae) control measures available to commercial producers of any flower crop in Florida.

\begin{tabular}{|c|c|c|c|}
\hline Common Name & Trade Name/Formulation & $\begin{array}{l}\text { Production } \\
\text { Site }^{1}\end{array}$ & Notes from Label $^{2}$ \\
\hline Pyridaben & Sanmite & $F, G$ & $\begin{array}{l}\text { Maximum seasonal use rate: Do not exceed } \\
21.34 \text { ounces per acre per year. }\end{array}$ \\
\hline Pyriproxyfen & Distance IGR & $F, G$ & $\begin{array}{l}\text { Greenhouse, silverleaf \& sweetpotato } \\
\text { whitefly; for foliar spray application, apply } \\
\text { Distance no more than two times per } \\
\text { cropping cycle or no more than two times } \\
\text { per } 6 \text { months. For sprench application, if a } \\
\text { second application is needed, allow a } \\
\text { minimum of } 21 \text { days between applications. } \\
\text { For drench application: Do not drench } \\
\text { plants more than one time per crop cycle. } \\
\text { Phytotoxicity has been observed on salvia } \\
\text { (Salvia spp.), ghost plant (Graptopetalum } \\
\text { paraguayense), schefflera (Schefflera spp.), } \\
\text { gardenia (Gardenia spp.), and coral bells } \\
\text { (Heuchera sanguinea). It is therefore } \\
\text { recommended that Distance not be used on } \\
\text { these plants. Do not apply to poinsettia } \\
\text { after bract formation. }\end{array}$ \\
\hline \multirow[t]{2}{*}{$\begin{array}{l}\text { Refined Petroleum } \\
\text { Distillate }\end{array}$} & Saf-T-Side Spray Oil & $\mathrm{F}$ & $\begin{array}{l}\text { This insecticide's mode of action is through } \\
\text { suffocation of eggs, larvae and nymphs of } \\
\text { insects and adults of soft bodied insects. } \\
\text { This mode of action necessitates total spray } \\
\text { coverage. Bedding plants; do not use in } \\
\text { combination with or immediately before or } \\
\text { after spraying with fungicides such as } \\
\text { captan, oxythioquinox or any product } \\
\text { containing sulfur. Also do not use with } \\
\text { carbaryl (Sevin) or dimethoate. Do not use } \\
\text { with any product whose label recommends } \\
\text { the use of no oils. Do not use in } \\
\text { combination with NPK foliar fertilizer } \\
\text { applications. }\end{array}$ \\
\hline & Ultra-Fine Oil & $\mathrm{F}$ & $\begin{array}{l}\text { Caution: Spray no more than } 4 \text { consecutive } \\
\text { sprays. The frequency of consecutive } \\
\text { sprays should not exceed once every } 2 \\
\text { weeks. Early morning applications are } \\
\text { recommended. Do not tank mix with } \\
\text { insecticide or miticide whose label indicates } \\
\text { that it should not be used with oil. Do not } \\
\text { use with captan, chlorothalonil, dimethoate, } \\
\text { methiocarb, oxythioquinox, propargite, or } \\
\text { any product containing sulfur. This list is not } \\
\text { exhaustive; therefore for products not } \\
\text { included in this list consult label for } \\
\text { compatibility information. }\end{array}$ \\
\hline
\end{tabular}


Pesticide Options for Important Insect, Mite and Mollusk Pests of Commercial Flowers in....

Table 21. Whitefly (Homoptera: Aleyrodidae) control measures available to commercial producers of any flower crop in Florida.

\begin{tabular}{|c|c|c|c|}
\hline Common Name & Trade Name/Formulation & $\begin{array}{l}\text { Production } \\
\text { Site }^{1}\end{array}$ & Notes from Label ${ }^{2}$ \\
\hline S-Kinoprene & Enstar II & $G$ & $\begin{array}{l}\text { Application should be made to poinsettia } \\
\text { before bract formation. Foliar damage on } \\
\text { some sensitive varieties can result. Some } \\
\text { varieties of roses, such as Yellow Blooded } \\
\text { roses, show delayed damage. Slight to } \\
\text { moderate injury has occurred on some } \\
\text { blooms under certain conditions, suggest } \\
\text { application be made in pre-bloom stage. }\end{array}$ \\
\hline Tau-Fluvalinate & Mavrik Aquaflow & $F, G$ & $\begin{array}{l}\text { Repeat applications not more than four } \\
\text { times per month at } 5-7 \text { day intervals unless } \\
\text { otherwise noted in use directions. }\end{array}$ \\
\hline Thiamethoxam & Flagship 25WG & $F, G$ & $\begin{array}{l}\text { Shade house, container \& nursery grown } \\
\text { (F); do not exceed } 8 \text { oz. per acre per crop or } \\
\text { year, which ever is shorter. }\end{array}$ \\
\hline \multicolumn{4}{|c|}{$\begin{array}{l}\text { Footnotes: } \\
1 \text { "F" indicates field production. "G" indicates greenhouse production. } \\
{ }^{2} \text { Notes are taken from product labels and restrict use to the condition indicated (suppression, beet armyworm, exposed } \\
\text { thrips, etc.), limit numbers or patterns of applications, provide phytotoxicity precautions, etc. } \\
{ }^{3} \text { The combination of acephate with a pyrethroid, such as fenpropathrin, has resulted in synergistic effects on whitefly } \\
\text { mortality. }\end{array}$} \\
\hline
\end{tabular}

